PAPER

\section{Dynamics and stability of divertor detachment in $\mathrm{H}$-mode plasmas on JET}

To cite this article: A R Field et al 2017 Plasma Phys. Control. Fusion 59095003

View the article online for updates and enhancements.

\section{Related content} Maior results from the stellarator

Contrasting $\mathrm{H}$-mode behaviour with deuterium fuelling and nitrogen seeding in the all-carbon and metallic versions of JET

- Integration of a radiative divertor for heat load control into JET high trianqularity ELMy H-mode plasmas

\section{Recent citations}

- The dependence of exhaust power
$\frac{\text { components on edge gradients in JET-C }}{\text { and JET-ILW H-mode plasmas }}$
A R Field et al
- Self-sustained divertor oscillations in
$\frac{\text { ASDEX Upgrade }}{\text { P. Heinrich et al }}$
- Spectroscopic investigation of $N$ and Ne
seeded induced detachment in JET ITER-
like wall L-modes combining experiment
and EDGE2D modeling
B. Lomanowski et al




\title{
Dynamics and stability of divertor detachment in H-mode plasmas on JET
}

\author{
A R Field ${ }^{1}$, I Balboa ${ }^{1}$, P Drewelow $^{2}$, J Flanagan ${ }^{1}\left(\mathbb{0}\right.$, C Guillemaut $^{1}$, \\ J R Harrison ${ }^{1}$, A Huber ${ }^{3}$, V Huber ${ }^{4}$, B Lipschultz ${ }^{5}{ }^{\oplus}$, G Matthews ${ }^{1}$, A Meigs ${ }^{1}$, \\ J Schmitz $^{5}$, M Stamp ${ }^{1}$, N Walkden ${ }^{1}$ and JET contributors ${ }^{6}$ \\ EUROfusion Consortium, JET, Culham Science Centre, Abingdon, OX14 3DB, United Kingdom \\ ${ }^{1}$ CCFE, Culham Science Centre, Abingdon, Oxon, OX14 3DB, United Kingdom \\ ${ }^{2}$ Max-Planck-Institut für Plasmaphysik, Teilinsitut Greifswald, D-17491 Greifswald, Germany \\ ${ }^{3}$ Forschungszentrum Jülich GmbH, Institut für Energie- und Klimaforschung_Plasmaphysik, D-52425 \\ Jülich, Germany \\ ${ }^{4}$ Supercomputing Centre, Forschungszentrum Jülich GmbH, D-52425 Jülich, Germany \\ ${ }^{5}$ York Plasma Institute, University of York, Heslington, York, YO10 5DD, United Kingdom \\ E-mail: anthony.field@ukaea.uk
}

Received 21 March 2017, revised 19 May 2017

Accepted for publication 1 June 2017

Published 5 July 2017

\begin{abstract}
The dynamics and stability of divertor detachment in $\mathrm{N}_{2}$ seeded, type-I, ELMy H-mode plasmas with dominant NBI heating in the JET ITER-like wall device is studied by means of an integrated analysis of diagnostic data from several systems, classifying data relative to the ELM times. It is thereby possible to study the response of the detachment evolution to the control parameters (SOL input power, upstream density and impurity fraction) prevailing during the inter-ELM periods and the effect of ELMs on the detached divertor. A relatively comprehensive overview is achieved, including the interaction with the targets at various stages of the ELM cycle, the role of ELMs in affecting the detachment process and the overall performance of the scenario. The results are consistent with previous studies in devices with an ITER-like, metal wall, with the important advance of distinguishing data from intra- and inter-ELM periods. Operation without significant degradation of the core confinement can be sustained in the presence of strong radiation from the $\mathrm{x}$-point region (MARFE).
\end{abstract}

Keywords: divertor, H-mode, detachment, JET-ILW, stability, evolution, ELM

(Some figures may appear in colour only in the online journal)

\section{Introduction}

Operation of ITER in H-mode with a tolerable, inter-ELM target heat flux $\left(q_{t}<10 \mathrm{MW} \mathrm{m}^{-2}\right)$ will require enhanced radiation and a partially detached divertor solution, while tolerable ELM energy deposition will also necessitate mitigation measures (RMPs or ELM pacing) or operation in a small-ELM regime [1]. To achieve divertor detachment requires either radiation from seeded or intrinsic impurities or operation at high enough upstream density for the SOL

\footnotetext{
6 See the author list of Overview of the JET results in support to ITER by X Litaudon et al to be published in Nuclear Fusion Special issue: overview and summary reports from the 26th Fusion Energy Conference (Kyoto, Japan, 17-22 October 2016).
}

plasma to be sufficiently cool for recombination to occur. The momentum of the SOL plasma can then be transfered to the wall by collisions with neutrals, thereby reducing the direct ion flux and deposited power incident on the targets $[2,3]$. In order to understand and control the operation of detached, $\mathrm{H}$-mode plasmas, it is important to study their behaviour in terms of the evolution and stability of the detached state in response to changes in control parameters, e.g. the impurity seeding rate or heating power, the interaction of ELMs with the scrape-off layer (SOL) plasma and divertor and the effect of detachment on core confinement. Here, we present such an investigation of $\mathrm{N}_{2}$ seeded, type-I ELMy H-mode plasmas exhibiting divertor detachment in the JET device with the ITER-like wall (ILW) [4]. 
Evolution from an attached to a detached state (or vice versa) is most easily studied in L-mode plasmas with density or power ramps, which evolve gradually, without the complication of the ELM energy efflux burning through the detached plasma [5]. It is, however, important to study detachment in $\mathrm{H}$-mode plasmas because the baseline scenario for ITER operation at high fusion gain $\left(Q_{\mathrm{DT}} \geqslant 10\right)$ is a seeded, type-I ELMy H-mode at a high Greenwald density fraction $\left(f_{\mathrm{GW}}\left(=\overline{n_{e}} / n_{e, \mathrm{GW}}\right) \sim 0.85\right.$ [6] $)$ [1]. Such studies require careful analysis, distinguishing data from the interand intra-ELM periods, if measurements are to be correctly interpreted, e.g. in terms of SOL power balance, target power deposition, detachment evolution and burn-through or 'buffering' of the ELM power loading by the divertor plasma [7]. It is not possible to provide a comprehensive review of relevant detachment studies here, so below we summarise the main results from two recent studies on JET and ASDEX-U, both devices having an ITER-like metal (W and Be) first wall.

We believe that there are two novel features to the analysis presented here: firstly, the ELM energy losses from the confined plasma $\Delta W_{\text {ELM }}$ are determined and suitably timeaveraged, enabling the input power to the divertor region during the inter-ELM periods $P_{\mathrm{Div}}^{\mathrm{i}-\mathrm{ELM}}$ to be determined-a quantity which is essential for comparisons with theoretical predictions and results of numerical modelling. Secondly, wherever possible, we classify (and integrate or average) all of the data as from inter- or intra-ELM periods, thereby allowing the physics of the inter-ELM detachment evolution to be unravelled from the perturbing effect of the ELMs. To do this, we have developed a suite of object-oriented Python codes for each of the divertor diagnostics and the high-resolution Thomson scattering system (HRTS) [8], including pedestal profile fitting, which enable the analysed data to be combined easily. Although we do not make comparisons with results of numerical simulations here, we believe that this process has facilitated advances in our understanding, e.g. being able to relate the observed detachment behaviour to the closeness of the divertor input power to the thresold power for initiation of detachment.

An issue which complicates our analysis arises from the investigation presented in [9], in which a deficiency of up to $25 \%$ in the overall energy balance of JET-ILW pulses is found between the input heating energy and the sum of the total radiation from bolometry and deposited energy measured by target and limiter calorimetry. Insufficient data was available to determine whether this arises from a shortfall in the input power (Ohmic, NBI and ICRH) or some un-measured loss mechanism. Here, we have attempted to take this potential deficit into account in our analyses.

\subsection{Previous studies of divertor detachment in JET and ASDEX-U}

A comparison of detachment and density limit behaviour in JET with the carbon-dominated (CFC) and the ITER-like wall, is presented in [5] for both L- and H-mode pulses. These experiments were performed without impurity seeding with a much higher $D_{2}$ fuelling rate than the experiments reported here, i.e. $\Gamma_{\mathrm{D}_{2}} \leqslant 2 \times 10^{23} \mathrm{e} \mathrm{s}^{-1}$ see $\leqslant 2.2 \times 10^{22} \mathrm{e} \mathrm{s}^{-1}$. The L-mode, density ramp pulses exhibited an evolution to full detachment and formation of an X-point MARFE ${ }^{7}$. With the ILW a longer period of stable operation with full detachment, or even with the x-point MARFE, was possible than with the CFC wall, this period increasing with input power. The level of total radiation $P_{\mathrm{Rad}}^{\text {tot }}$ was found to be correlated with the density limit. With the CFC wall, the fraction of radiated power $f_{\text {Rad }}^{\text {tot }}=P_{\text {Rad }}^{\text {tot }} / P_{1, \text { th }}$ (where $P_{1, \text { th }}$ is the net loss power crossing the separatrix) increased more quickly with the lineaveraged density than in pulses with the ILW. In both cases, the $x$-point MARFE occured at approximately the same power crossing the separatrix, $P_{\mathrm{Sep}}=P_{1, \text { th }}-P_{\mathrm{Rad}}^{\mathrm{Pl}}$, where $P_{\mathrm{Rad}}^{\mathrm{Pl}}$ is the total radiation from the confined plasma.

With the higher fuelling rate, the H-mode pulses reported in [5] exhibit a transition from type-I to small, 'grassy' ELMs and eventually a back transition to L-mode, which is an effective H-mode density limit. Without impurity seeding, the radiation fraction $f_{\mathrm{Rad}}^{\text {tot }} \lesssim 0.45$ was less than in the experiments reported here, however, the transition to small ELMs resulted in a cooler, denser pedestal and consequently a significant degredation in core confinement, with the confinement enhancement factor $H_{98, y}$ decreasing by $\sim 20 \%$ to $\sim 0.7 \%$ at the density limit. Spectroscopic imaging of the divertor using a multiple-wavelength, filtered camera system [10] was used to study the evolution to detachment but no attempt was made to distinguish data with and without ELMs.

Experimental studies of $\mathrm{N}_{2}$ seeded, detached H-mode operation at high radiated power fraction $f_{\mathrm{Rad}}^{\text {tot }} \lesssim 0.85$ have been performed on ASDEX-U [11, 12], together with complementary comparisons with numerical SOL modelling results. These experiments were performed with $8.2 \mathrm{MW}$ total input power, twice the $\mathrm{D}_{2}$ fuelling rate used in our JET experiments $\left(\Gamma_{\mathrm{D}_{2}} \sim 2 \times 10^{22} \mathrm{e} \mathrm{s}^{-1}\right)$ and an equal, constant $\mathrm{N}_{2}$ puffing rate. The detachment was found to evolve through four phases: I-with detached inner, high-field-side (HFS) target (without seeding) and attached outer, low-field-side (LFS) target; II-with $6-8 \mathrm{kHz}$ radiative fluctuations near X-point ; III-with partially detached LFS target (detached only near strike point) during the inter-ELM periods and a reduction in ELM frequency, after which a stronly radiating zone appears near the x-point; IV-complete detachment. During the latter phase, the particle and power fluxes to the targets drop to $\lesssim 5 \times 10^{22} \mathrm{~s}^{-1}$ and $\lesssim 0.5 \mathrm{MW} \mathrm{m}^{-2}$ respectively, while the $\mathrm{x}$-point radiation exhibits Balmer $\mathrm{D}_{\delta}$ and NIII line radiation, which indicates the presence of a cold $\left(T_{e} \lesssim 5 \mathrm{eV}\right)$ recombining region there. There is also a concommittant reduction of pedestal pressure by $40 \%-50 \%$ with a strong parallel temperature gradient, while the core confinement was only reduced by $\sim 10 \%$ with $H_{98, y}$ remaining $\gtrsim 0.9$.

\subsection{Theory of detachment stability and control}

The stability of detached divertors is treated in [13] in terms of a 1D analytic model of parallel thermal conduction in full

\footnotetext{
7 An x-point MARFE is a strongly radiating, cold, recombining region of the confined plasma in the vicinity of the $\mathrm{x}$-point.
} 
finite-aspect-ratio geometry. With the heating of the SOL from radial thermal transport across the separatrix localised to the main chamber and the radiation localised to the divertor, where the impurity radiation peaks over a limited temperature range defined by coronal equilibrium, the resulting temperature profile exhibits a region of steep gradient and strong radiation termed the 'thermal front'. Below this front, the temperature is reduced to a few $\mathrm{eV}$ at which recombination can occur. Note that such analytic models assume that a stable solution exists. Also, presence of a thermal front is a necessary but not sufficient condition for recombination to occur.

A stable front requires equlibrium between radiation loss inside the front $q_{f}$ and the heat input at the hot side of the front $q_{i}$. If this equilibrium is violated then the front will move: either towards $\left(\left|q_{i}\right|>\left|q_{f}\right|\right)$ or away from the target $\left(\left|q_{i}\right|<\left|q_{f}\right|\right)$, stability of the front location requiring that ${ }^{8} \mathrm{~d} / \mathrm{d} z\left(q_{i}-q_{f}\right)<0$. The principal parameters which control the divertor detachment are: the upstream density $n_{u}$, the impurity concentration $f_{I}=n_{I} / n_{e}$ and the power crossing the separatrix into the divertor region, $P_{\mathrm{Div}}^{\text {tot }}$. The detachment 'window' in these parameters $C=\left\{n_{u}, f_{I}, P_{\mathrm{Div}}^{\mathrm{tot}}\right\}$ is defined as the change in the parameter required to move the front from the target to the $\mathrm{x}$-point.

The sensitivity of the front location to the control parameters is derived in [14], which extends the analysis of [13], taking account of the spatial variation in field magnitude $|B|$. This variation affects the stability through the $1 / B^{2}$ dependence of the effective thermal conductivity $\kappa=\kappa_{\|}\left(\frac{B_{\times}}{B_{t}}\right)^{2}$ (where $B_{\times}$is the field at the x-point and $B_{t}$ the field at the target), which helps to stabilise the front to a region of larger major radius. For the JET V5 equilibrium shown in figure A1 (and for the flux surface at normalised poloidal flux $\left.\psi_{N}=1.001\right)$ at the LFS $\frac{B_{\times}}{B_{t}} \sim 1.05$, which is weakly stabilising, while at the HFS, the field decreases towards the x-point $\left(\frac{B_{x}}{B_{t}} \sim 0.96\right)$, encouraging the front to move away from the target.

Within the framework of this theory, according to equation (27) of [14], the position of the thermal front is a function of a combination of the three control parameters, henceforth referred to as the 'detachment' factor, defined as $\mathcal{F}_{\text {det }}=n_{u} f_{I}^{1 / 2} / p_{\text {Div }}^{5 / 7}$. The detachment windows in any one parameter is determined by holding the other two constant and determining the change of the desired parameter to move the front from the target to the x-point. Note that the expression for the window in $\mathcal{F}_{\text {det }}$ is the same as that for the upstream density $n_{u}$ alone.

The fractional detachment windows are defined as $\Delta \tilde{C}=C_{x} / C_{t}-1$, where $C_{x} / C_{t}$ is given by equation (30) of [14]. Numerically, for the flux surface considered above, $\Delta \tilde{C} \sim\{0.3,0.67,-0.3\}$ for the LFS divertor leg, i.e. there is a larger fractional window in impurity concentration than density, while an increase in power moves the front closer to the target. For the HFS leg the windows are smaller

\footnotetext{
8 Here, the normalised parallel distance $z$ is defined by $\mathrm{d} z=\frac{B_{\times}}{B} \mathrm{~d} l$, where $B_{\times}$ is the value of the field at the $\mathrm{x}$-point, $\mathrm{d} l$ is an element of parallel field line length and $z=0$ at the target.
}

$\{0.14,0.31,-0.17\}$ because of the destabilising effect of the field variation. The detachment windows in $\mathcal{F}_{\text {det }}$ are hence 0.3 and 0.14 for the LFS and HFS divertor legs respectively. Hence, following the initial onset of detachment, changes in these parameters of only a few $10 \%$ are expected to move the thermal front along the full length of the divertor legs.

\subsection{Structure of paper}

The structure of the remaining sections of this paper is as follows: section 2 describes the $\mathrm{N}_{2}$ seeded type-I ELMy $\mathrm{H}$-mode discharge scenario used for these investigations; the evolution of the divertor from an attached to a detached state for an example pulse \#89241 with an increasing level of seeding, including: the localisation of the radiation and recombination fronts using spectroscopic imaging data; the ELM energy losses from the confined plasma, total radiation and deposited energy on the LFS divertor target during the ELMs; and the interaction of the SOL plasma with the divertor targets at various stages of the ELM cycle.

An analysis of the conditions promoting divertor detachment during the inter-ELM periods is presented in section 3. The evolution of the degree of detachment (DoD) during the ELM cycle is first analysed, including its dependence on the pedestal pressure and pressure gradient and its dependence on electron temperature and density at the pedestal top and at the separatrix, the latter being determined from consideration of separatrix power balance. The dependencies of the DoD on the power into the divertor region during the inter-ELM periods $P_{\text {Div }}^{\mathrm{i}-\mathrm{ELM}}$, which has to be determined accounting for the time-averaged ELM losses, and on the level of $\mathrm{N}_{2}$ seeding are also investigated.

In unseeded pulses, at lower values of $f_{\mathrm{GW}} \lesssim 0.75$, at the heating power used for these experiments, $P_{\mathrm{Div}}^{\mathrm{i}-\mathrm{ELM}}$ is found to be close to the threshold for divertor detachment. Under these conditions, rapid oscillations of the divertor plasma from an attached to a detached state are observed, as have been reported previously on JET [15] and ASDEX-U [16]. This phenomenon is investigated in more detail in section 4 .

In section 5 the evolution of pulse \#89244 in which the heating power is increased during a detached phase is presented, including: analysis of the dependence of the DoD on the divertor input power during the inter-ELM periods $P_{\text {Div }}^{\text {i-ELM }}$ and the radiated power from the divertor; the evolution of the total radiation distributions and the thermal front and recombination region; and the behaviour of the inter-ELM averaged target $j_{\text {sat }}$ profiles, which show that at the higher power the SOL plasma only partially re-attaches to the HFS target, while the LFS target remains detached. Note that this is not a clear demonstration of power hysteresis of detachment because the influx of seeded $\mathrm{N}_{2}$ was constantly increasing throughout this pulse.

With the aim of understanding the effect of the ELMs on the SOL plasma in more detail, the behaviour of the radiated power distributions at intervals through the ELM cycle during the attached phase of pulse \#89244are presented in section 6.

We present a discussion of the main results from each of the main sections in section 7 , with the aim of bringing all of 
the relevant results together, interpreting these in terms of the evolution of detachment and the radiation from seeded and ELM sputtered impurities in the divertor plasma. Finally, in section 8 we present the main conclusions of this work.

\section{Experiments}

In this section, the $\mathrm{N}_{2}$ seeded type-I ELMy $\mathrm{H}$-mode discharge scenario and magnetic configuration are described in section 2.1, followed by a description in section 2.2 of the overall evolution of an example pulse with ramped $\mathrm{N}_{2}$ seeding, which exhibits a gradual transition of the divertor conditions from an attached to a fully detached state. The consequent evolution of the radiation, ionisation and recombination fronts in the divertor plasma are described in section 2.3. Total radiation distributions are obtained from tomographic reconstructions of multi-chord bolometer data, whilst the ionisation and recombination fronts are determined from $\mathrm{N}$ II, $\mathrm{D}_{\alpha}$ and $\mathrm{D}_{\gamma}$ spectral line emissivity distributions measured using filtered camera systems. Changes to the ionisation front location due to ELMs, inferred by subtracting NII emission distributions from successive frames with and without ELMs, are discussed in section 2.4. In section 2.5 the ELM energy losses are quantified, considering the total energy losses from the confined plasma $\Delta W_{\mathrm{ELM}}$, the total radiation during the ELMs $\Delta W_{\mathrm{Rad}}^{\mathrm{ELM}}$ and the deposited energy onto the lower horizontal (T5) divertor target $\Delta W_{\text {Dep }}^{\mathrm{T} 5}$. Finally, the interaction of the SOL plasma with the targets at various stages of the ELM cycle in terms of ELM-cycle averaged $j_{\text {sat }}$ profiles is described in section 2.7 , including their variation with the level of impurity seeding.

\subsection{Type-I, ELMy H-mode discharge scenario}

The experiments were performed using a single-null diverted magnetic configuration (V5) with the LFS strike point on the horizontal T5/C target tile and the HFS strike point on the vertical T3 tile. (The divertor target geometry is shown in figure A1 of appendix A.) Pulses were run at a plasma current $I_{p}$ of $2 \mathrm{MA}$ at $B_{t}$ of $2.2 \mathrm{~T}$ with $8-15 \mathrm{MW}$ of input power with a combination of Ohmic, NBI and ICRH heating, which is sufficient for the pulses to enter the type-I ELMy $\mathrm{H}$-mode confinement regime. The analysis presented here is of eight pulses with $\mathrm{D}_{2}$ fuelling at $1-1.3 \times 10^{22} \mathrm{e} \mathrm{s}^{-1}$ (plus $\mathrm{H}_{2}$ minority for ICRH). This results in densities in the range $\overline{n_{e}} \sim 5-7 \times 10^{19} \mathrm{~m}^{-3}$ corresponding to Greenwald density fractions of $f_{\mathrm{GW}}\left(=\bar{n}_{e} / n_{e, \mathrm{GW}}\right) \sim 0.7-0.95$, where $n_{e, \mathrm{GW}}\left[10^{20} \mathrm{~m}^{-3}\right]=\mathrm{I}_{p}[\mathrm{MA}] /\left(\pi a^{2}\right)$ and $a$ is the plasma minor radius in $m$ [6]. Of the pulses analysed here, one was an unseeded reference (\#89238), while others (\#89239-41, ..44, ..46-48) were seeded with $\mathrm{N}_{2}$ at various rates in the range $0.4-2.2 \times 10^{22} \mathrm{e} \mathrm{s}^{-1}$. The seeding gas was introduced from an annular gas-inlet manifold (GIM-9) in the lower divertor close to the outer edge of the horizontal target (T5).

\subsection{Overview of pulse \#89241 with ramped seeding}

The evolution of an example pulse \#89241 with $P_{\mathrm{NB}} \sim 7.5 \mathrm{MW}, P_{\mathrm{ICRH}} \sim 2.1 \mathrm{MW}$ and $\overline{n_{e}} \lesssim 6.5 \times 10^{19} \mathrm{~m}^{-3}$, in which the $\mathrm{N}_{2}$ seeding rate was gradually ramped from 0.7 to $2.2 \times 10^{22} \mathrm{e} \mathrm{s}^{-1}$, is shown in figure 1 . Later in the pulse, after $52 \mathrm{~s}$, when the nitrogen concentration is sufficient, there is a step decrease of the ELM frequency and the lineaveraged density (quantified in terms of the Greenwald density fraction) increases from $f_{\mathrm{GW}} \sim 0.8$ to 0.95 . Initially, when the divertor plasma is attached to the targets, the fraction of power radiated from the confined plasma during the inter-ELM periods $f_{\mathrm{Rad}}^{\mathrm{i} \text {-ELM }} \sim 40 \%$, while after the ELM frequency drops, this increases to $\sim 60 \%$. An analysis of the power blance presented in section 3 highlights the importance of the core radiation in determining the conditions favouring inter-ELM divertor detachment. Throughout the pulse, the $\mathrm{H}$-mode confinement enhancement factor $H_{98, y} \sim 0.8$ [17], shown in figure $1(\mathrm{c})$, remains quite constant.

The onset of detachment results in a reduction in the total ion fluxes $\Gamma_{i}^{\text {tot }}$ to the divertor targets measured using fixed Langmuir probes (LPs) ${ }^{9}$. During the inter-ELM periods, when the divertor is attached to the target, the total ion fluxes are typically $\Gamma_{i}^{\text {tot }} \sim 2 \times 10^{23} \mathrm{~s}^{-1}$ and $4 \times 10^{23} \mathrm{~s}^{-1}$ to the LFS and HFS targets respectively. It can be seen from figures 1(e) and (f) that after $52 \mathrm{~s}$ there is a gradual decrease of $\Gamma_{i}^{\text {tot }}$ to $\lesssim 20 \%$ of these values as the divertor plasma detaches from both of the targets.

The (DoD) is often quantified as the ratio of the ion flux to the target from a two-point SOL model scaling to the measured value, i.e. $\operatorname{DoD}=\Gamma_{i}^{\mathrm{scal}} / \Gamma_{i}^{\mathrm{meas}}$, where $\Gamma_{i}^{\mathrm{scal}}=$ $C_{\text {DoD }} n_{e \text {,sep }}^{2} / T_{e \text {,sep }}^{3 / 2}$ and $n_{e \text {,sep }}$ and $T_{e \text {,sep }}$ are the upstream electron density and temperature at the mid-plane separatrix $[18]^{10}$. The value of the parameter $C_{\mathrm{DoD}}$ can be determined by normalising $\Gamma_{i}^{\text {scal }}$ to the measured value during the attached phase of the pulse. The DoD parameter is shown below in figures $8(e)$ and (f) is calculated using the total ion fluxes to targets, from which it can be seen that this increases to $\sim 5$ at the LFS and $\sim 8$ at the HFS by the end of the pulse.

\subsection{Evolution of radiation, ionisation and recombination fronts}

The evolution of the radiation, ionisation and recombination fronts during the gradual progression from an attached to fully detached state is diagnosed using multi-channel bolometry and multi-wavelength, visible spectral imaging systems. The expected behaviour of the SOL plasma during the detachment process is found: radiative cooling over an extended region of the SOL, an ionisation front localised through the presence of low ionisation stages of the seeded impurity, followed by a region of cold, recombining plasma extending fom the

9 This is calculated from integration of the ion saturation currents $j_{\text {sat }}$ measured over arrays of probes in either the LFS or HFS targets, where the LFS target comprises the inclined tile (T5), the floor tile (T6) and the two vertical targets (lower T7 and upper T8) and the HFS target the two vertical tiles (upper T1 and lower T3) and the floor tile (T4).

10 Note that this definition of the DoD is equivalent to DoD ${ }^{\text {int }}$, the integral value referred to in [5], rather than DoD peak determined from $j_{\text {sat }}$ at the strike point, which is typically considerably greater. 

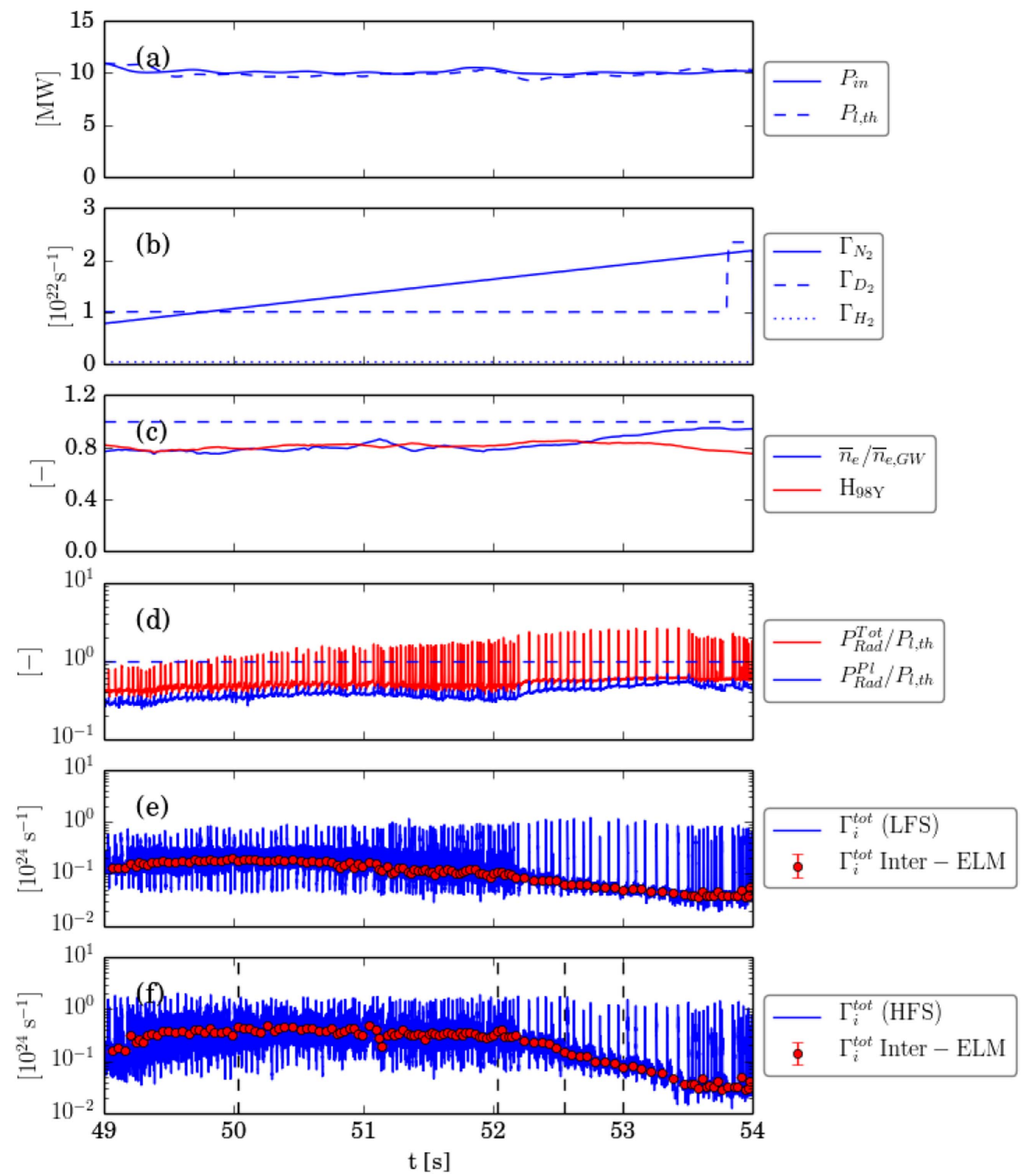

Figure 1. The evolution of JET pulse \#89241 showing: (a) total input power $P_{\text {in }}$ (solid) and the thermal loss power $P_{1, \text { th }}$ (dashed); (b) the $\mathrm{N}_{2}$ seeding rate $\Gamma_{\mathrm{N}_{2}}$ (solid) and the $\mathrm{D}_{2}$ and $\mathrm{H}_{2}$ fuelling rates $\Gamma_{\mathrm{D}_{2}}$ (dashed) and $\Gamma_{\mathrm{H}_{2}}$ (dotted); (c) the Greenwald density fraction $f_{\mathrm{GW}}$ and the H-mode confinement enhancement factor $H_{98, y}$; (d) the fraction of $P_{1, \text { th }}$ radiated in total $f_{\mathrm{Rad}}^{\text {tot }}=P_{\mathrm{Rad}}^{\text {tot }} / P_{1, \text { th }}$ (red) and from the confined plasma $f_{\mathrm{Rad}}^{\mathrm{Pl}}=P_{\mathrm{Rad}}^{\mathrm{Pl}} / P_{1, \text { th }}$ (blue); (e), (f) the total ion fluxes $\Gamma_{i}^{\text {tot }}$ to the LFS (tiles \#2-4) and HFS (tiles \#5-8) targets respectively (blue), with interELM averaged values (red). The times of the emissivty distributions shown in figure 2 are shown by the vertical dashed lines.

ionisation front to the target. As the detachment deepens, the recombination zone moves up the SOL from the target surface, increasing in extent towards the x-point, while the ionisation front and radiating zone expand similarly upwards, finally extending above the x-point with significant radiation within the confined plasma periphery at the deepest DoD, a MARFE forming in the $x$-point region in this final, fullydetached state.

2.3.1. Total radiation distributions: Distributions of the total radiated emissivity $\epsilon_{\text {Rad }}(R, z)$ are available from tomographic inversions [21, 22] of the data from a multi-channel, resistive bolometer system (KB5) [23]. Such distributions at the four times indicated in figure 1(f), which are selected during inter-ELM periods, are shown in figure 2. During the early, attached phase ( $\sim 50 \mathrm{~s})$ a distinct zone of emission can be observed in the LFS divertor leg, although emission from the $\mathrm{x}$-point region, just inside the separatrix dominates ${ }^{11}$. Already at the onset of the detachment phase $(\sim 52 \mathrm{~s})$, this emission begins to recede up the divertor leg towards the $\mathrm{x}$-point. This progression continues $(\sim 52.5 \mathrm{~s})$ and the level of emission from the $\mathrm{x}$-point increases as the detachment deepens. At the final time-point $(\sim 53 \mathrm{~s})$, there is an intense zone of emission at the

11 Note that these tomographic reconstructions of total emissivity distributions $(\epsilon(R, Z))$ of the KB5 bolometer data do not resolve fine details of the distributions in the divertor region, although they do give a rough quantitative measure. This is because most of the channels of the divertor bolometers (KB3) are not operational. 

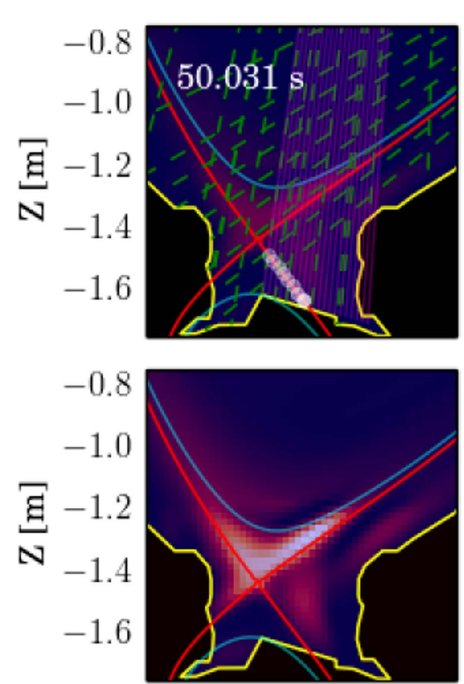

$\mathrm{R}[\mathrm{m}]$
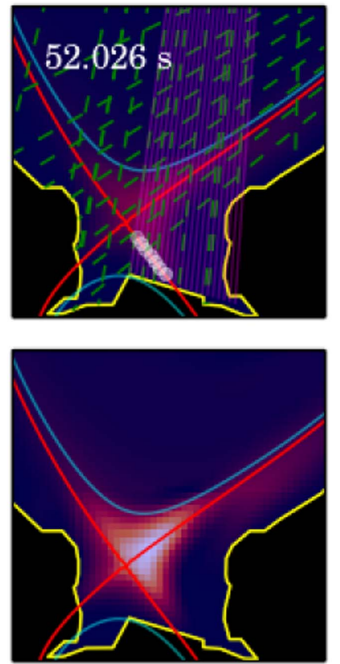

$\mathrm{R}[\mathrm{m}]$
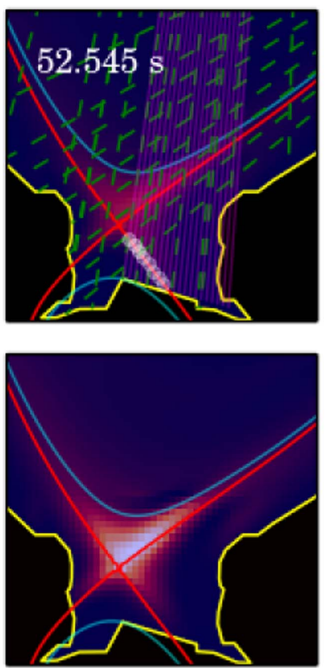

$\mathrm{R}[\mathrm{m}]$
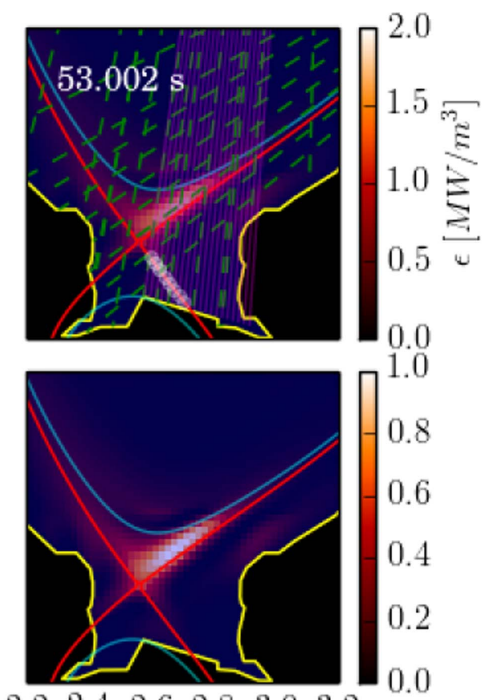

$\begin{array}{llllllll}2.2 & 2.4 & 2.6 & 2.8 & 3.0 & 3.2\end{array}$

$\mathrm{R}[\mathrm{m}]$

Figure 2. Distributions of the total radiated emissivity $\epsilon_{\operatorname{Rad}}(R, z)$ from bolometer tomography (BOLT) during pulse \#89241 at the times indicated in figure 1(f). In the top row the color scale representing the emissivities have the same normalisation, while in the bottom row each plot is normalised to its own maximum value. The smoothing time $\tau_{\mathrm{sm}}$ of the data is $5 \mathrm{~ms}$ and the times are selected during inter-ELM periods $\Delta t^{\mathrm{i}-\mathrm{ELM}} \geqslant 10 \mathrm{~ms}$. The lines of sight of the KB5 bolometer system are shown by the green-dashed lines. The opacity of the white markers (top) is linearly scaled to $\left\langle n_{e}^{\text {Stark }}\right\rangle_{\text {LoS }}: 0 \rightarrow 5 \times 10^{20} \mathrm{~m}^{-3}$ determined from the line-of-sight integrated profile broadening of the $\mathrm{D}_{10-2}$ line [19] measured by the KT3D spectrometer (20 lines of sight in magenta) [20]. The flux surface at $\psi_{N}=0.98$ is also indicated (cyan), as is the separatrix (red).

foot of the pedestal $\left(0.98<\psi_{N}<1.0\right)$ to the LFS of the $\mathrm{X}$-point.

Better quantitative appreciation of changes in the emissivity distribution can be obtained by calculating the integrated power radiated over various regions of the equilibrium. Results of such analysis are presented in figure 6(e), which shows the radiated power from: the whole vessel, the mantle region $\left(\rho_{N}>0.9\right.$ and $Z>Z_{\times}$, where $\left.\rho_{N}=\psi_{N}^{1 / 2}\right)$ and the divertor region $\left(Z \leqslant Z_{\times}\right.$, where $Z_{\times}$is the height the $\mathrm{x}$-point below the mid-plane). This data is from tomographic reconstructions performed during inter-ELM periods with a smoothing time $\tau_{\text {sm }}$ of $5 \mathrm{~ms}$. From this it can be seen that about $\sim 50 \%$ of $P_{\mathrm{Rad}}^{\mathrm{tot}} \sim 4 \mathrm{MW}$ is radiated from the mantle, while the radiation from the divertor is $\lesssim 10 \%$ of the total. About half of the radiation from the mantle is from the confined x-point region $\left(\rho_{N}=0.9-1.0,|\Delta \theta| \leqslant 20^{\circ}\right.$, where $\Delta \theta=\theta-\theta_{\times}$and $\theta_{\times}$is the poloidal angle of the x-point (not shown)). Following the onset of detachment, the radiation from the $x$-point increases, while that from the divertor decreases, which is consistent with the recession of the zone of divertor emission towards the x-point and the formation of an X-point MARFE, as observed in previous studies $[5,11,12]$.

2.3.2. Ionisation and recombination fronts. The localisation and movement of the ionisation front and recombination dominated region of the SOL plasma can be determined from 2D distributions of spectral line intensities recorded by the KL11 divertor imaging system [10]. This system has three filtered, intensified CCD cameras with coincident, tangential views of the divertor region. For these experiments, the cameras were fitted with filters encompassing: $\mathrm{D}_{\alpha}(656.1 \mathrm{~nm})$ (d), $\mathrm{D}_{\gamma}(433.9 \mathrm{~nm})(\mathrm{f}), \mathrm{N}$ II $(500.4 \mathrm{~nm})$ (e), with bandwidths of $\Delta \lambda=1.5,1.5$ and $1.64 \mathrm{~nm}$ respectively. Distributions of the 2D line emissivities $\epsilon_{\text {Rad }}(R, z)$ are obtained by tomographically inverting the intensity distributions, using the same method as employed in $[5,24]$ but employing the SART algorithm (Simultaneous Algebraic Reconstruction Technique) for matrix inversion [25]. Examples of the resulting distributions are shown for pulse \#89241 in figure 3, which shows images for the $\mathrm{D}_{\alpha}, \mathrm{D}_{\gamma}$ and $\mathrm{N}$ II line emissivities and the emissivity ratio $\mathrm{D}_{\gamma} / \mathrm{D}_{\alpha}$.

The ratio of the $D_{\gamma} / D_{\alpha}$ emissivity is sensitive to the presence of volume recombination. When only collisional excitation and recombination (radiative and three-body) are the only processes governing the level populations, as $T_{e}$ decreases from $2 \rightarrow 1 \mathrm{eV}$, this ratio increases strongly $\left(\epsilon_{\mathrm{D}_{\gamma}} / \epsilon_{\mathrm{D}_{\alpha}} \sim 0.02 \rightarrow 0.2 \text {, assuming } n_{0} / n_{e}=1\right)^{12}$ as recombination begins to dominate the population of the higher- $n$ levels. The thermal front can be similarly located from the $\mathrm{N}$ II emissivity distribution, which in coronal equlibrium has its peak abundance in the narrow temperature range $T_{e} \sim 1 \rightarrow 3 \mathrm{eV}$. Hence, the peak emission from this transition is to be expected in a region at the foot of the thermal front, just before the plasma is sufficiently cooled by radiation for recombination to dominate over ionisation.

In 16 bit mode, the KL11 cameras have a frame rate of $32.8 \mathrm{~Hz}$ (corresponding to an exposure time of $30.5 \mathrm{~ms}$ ), which is comparable to the ELM frequency in these pulses.

12 The temperature at which this transition occurs increases modestly with the relative neutral density $n_{0} / n_{e}$. 

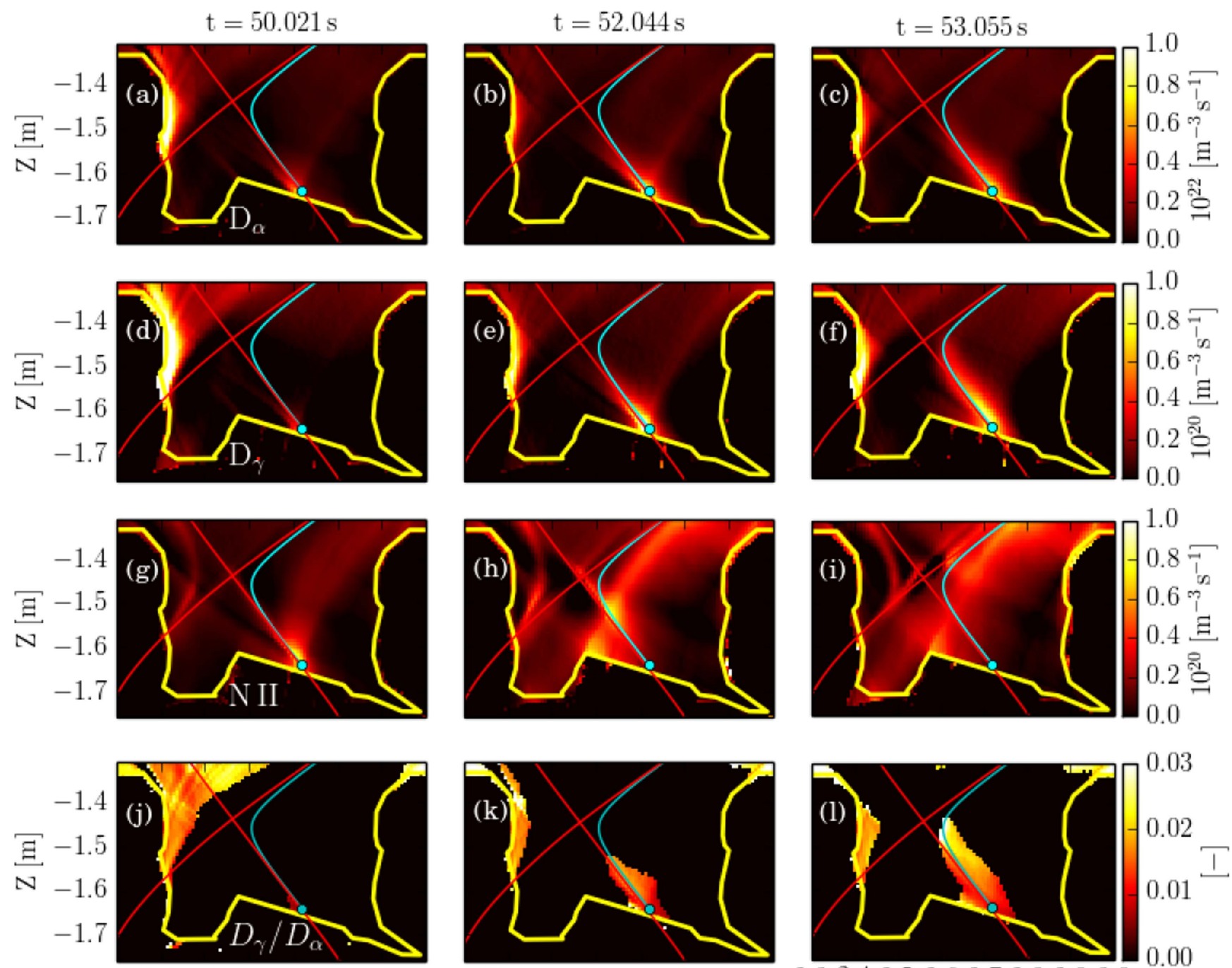

$$
\begin{gathered}
2.32 .42 .52 .62 .72 .82 .93 .02 .32 .42 .52 .62 .72 .82 .93 .02 .32 .42 .52 .62 .72 .82 .93 .0 \\
R[\mathrm{~m}]
\end{gathered}
$$

Figure 3. Distributions of line emissivities and emissivity ratios determined from tomographic inversions of filtered camera (KL11) data for: $\mathrm{D}_{\alpha}(656.1 \mathrm{~nm})(\mathrm{a})-(\mathrm{c}), \mathrm{D}_{\gamma}(433.9 \mathrm{~nm})(\mathrm{d})-(\mathrm{f}), \mathrm{N}$ II $(500.4 \mathrm{~nm})(\mathrm{g})-(\mathrm{i})$ and the emissivity ratio $\mathrm{D}_{\gamma} / \mathrm{D}_{\alpha}(\mathrm{j})-(\mathrm{l})$ in the divertor region for three frames during pulse \#89241 at times indicated in figure 4(e), which are selected to be ELM-free (blue). (The times indicated are at the start of the frames, which have exposures of $30.5 \mathrm{~ms}$.) The location of the separatrix is shown in red and the flux surface through the fixed Langmuir probe (S18C), just outside the separatrix is shown at the LFS (cyan-solid). In order to suppress spurious features, the $\mathrm{D}_{\gamma} / \mathrm{D}_{\alpha}$ ratio data is plotted only where it exceeds $2 \times 10^{-3}$ and the $\mathrm{D}_{\gamma}$ intensity also exceeds $2 \times 10^{19} \mathrm{~m}^{-3} \mathrm{~s}^{-1}$.

Hence, a large fraction of the frames record emission from one or more ELMs. In figure 3, data is shown for the first ELM-free frame after the times indicated in figure 1, which are during the attached phase (left), during the onset (middle) and end (right) of the transition to full detachment. These distributions are therefore characteristic of the inter-ELM periods, during which it can be seen that the frame-integrated $\mathrm{D}_{\alpha}$ and $\mathrm{D}_{\gamma}$ emission peaks close to the target during both the attached and detached phases. This perhaps surprising observation can be understood from the fact that, as discussed in section 2.6, the net ion fluence (time-integrated flux) to the targets during the inter-ELM periods are actually up to an order of magnitude larger than the fluences during the ELMs. This emission is hence due to recycling neutrals that are reionised near the strike point.
From the distributions of the N II emission (g)-(i), it can be seen that the ionisation front is initially close to the target during the attached phase $(\mathrm{g})$. The onset of detachment of the LFS target, indicated by the 'roll-over' in $\Gamma_{i}^{\text {tot }}$ already begins by $51 \mathrm{~s}$ (see figure 8(e)) and by $52 \mathrm{~s}$ (h) the ionisation front has already moved half way up the LFS divertor leg towards the x-point, while the detachment of the HFS divertor appears not to be so advanced. The apparent earlier detachment of the outer divertor might be because the $\mathrm{N}_{2}$ gas is puffed at the LFS of the divertor. By the time full detachment is achieved (53 s) (i) the ionisation front has moved to a region of the LFS SOL extending above the $\mathrm{x}$-point. Comparing this image to the last frame shown in figure 2 , it can be seen that the total radiation also peaks at a similar poloidal location at this time. 
From the $\mathrm{D}_{\gamma} / \mathrm{D}_{\alpha}$ distributions shown in figures 3(j)-(l), it can be seen that there is a region of the SOL between the radiation front and the target where this ratio is enhanced, indicating the presence of significant volume recombination. During the attached phase ( $\mathrm{j}$ ), although the maximum $\mathrm{D}_{\gamma} / \mathrm{D}_{\alpha}$ is located close to the target there is little volume recombination, most occurring at the target surface. At the later time $(\mathrm{k})$, there is a region with significant volume recombination below the radiation front, which is located about half way up the LFS divertor leg. In the fully detached state (l), there is stronger recombination in the vicinity of the x-point, while the radiation front extends further up the LFS SOL above the $\mathrm{x}$-point. It is more difficult to interpret the behaviour at the HFS divertor, where there appears always to be a cold, recombining region in front of the upper vertical target (T1).

The $\mathrm{D}_{\gamma} / \mathrm{D}_{\alpha}$ emissivity ratios shown in figures 3(j)-(1), typically lie in the range $\sim 0.005-0.02$, at or below the predicted ratio appropriate for collisional excitation alone, i.e. $\sim 0.02$ for $T_{e} \gtrsim 2 \mathrm{eV}$. The fact that the $\mathrm{D}_{\gamma} / \mathrm{D}_{\alpha}$ ratio is higher in the region of the SOL below the thermal front does imply that the $n=5$ population $\left(\mathrm{D}_{\gamma}(n=5-2)\right)$ is enhanced by recombination into the higher- $n$ levels there. Some other process must, however, be preferrentially populating the $n=3$ level, thereby enhancing the $\mathrm{D}_{\alpha}$ emissivity relative to that of $\mathrm{D}_{\gamma}$. In a detailed study of volume recombination and opacity effects in Alcator C-Mod [26], two possible processes were found to be significant in detached divertor plasmas. Firstly, self-absorption (optical thickness) of Lyman- $\beta$ populates the $n=3$ level from the ground state and, secondly, molecularly activated recombination (MAR) through collisions with vibrationally excited $\mathrm{D}_{2}$ molecules can preferentially populate the $n=2-4$ levels [3]. For some further discussion see section 7.1.

The dynamics of the inter-ELM detachment process at the LFS can be deduced by following the location of the maximum of these distributions along a particular flux surface in time. Results of such analyses are shown in figure 4, which show the profile evolution of the $\mathrm{D}_{\alpha}, \mathrm{D}_{\gamma}$ and $\mathrm{N}$ II emissivities and the $\mathrm{D}_{\gamma} / \mathrm{D}_{\alpha}$ ratio along the flux surface through one of the fixed target LPs (S18C) on T5 shown in figure 3 (cyan). The location of the profile maxima in the range $L_{\psi}=0.0 \rightarrow 0.3 \mathrm{~m}$, extending from the target to just above the x-point, where $L_{\psi}$ is the poloidal distance along the flux surface from the target is shown in each case, separately for the subset of frames encompassing ELMs (cyan) and free of ELMs (blue).

From the N II profiles ((c)-blue) it can be seen that the thermal front moves gradually away from the target soon after $50 \mathrm{~s}$, while the recombination front ((d)-blue) lags behind this, lying a few $\mathrm{cm}$ below the thermal front. After $51 \mathrm{~s}$, the thermal fronts remains for a while at a stable location $\sim 10 \mathrm{~cm}$ below the x-point. Following the drop in ELM frequency at $52.2 \mathrm{~s}$, which signifies formation of the x-point MARFE, both the thermal and recombination fronts jump to the vicinity of the x-point and the DoD deepens further.

The evolution of the detachment factor $\mathcal{F}_{\text {det }}$, which was introduced in section 1.2, is also shown in figure 4 ((e), blue). This has been calculated assuming that the $\mathrm{N}_{2}$ impurity fraction can be approximated by assuming $f_{I} \propto I_{\mathrm{NII}} / n_{u}$, i.e. by assuming that the influx is proportional to the NII intensity and that the concentration is proportional to the influx. In this case, because $n_{u}$ and $P_{\mathrm{Div}}^{\mathrm{i}-\mathrm{ELM}}$ are approximately constant, the change in $\mathcal{F}_{\text {det }}$ is dominated by the constantly increasing seeding level. The fractional change in $\mathcal{F}_{\text {det }}$ as the radiation front moves from the target $(\sim 0.3$ at $50 \mathrm{~s})$ to the x-point $(\sim 0.6$ at $50 \mathrm{~s})$ is approximately unity with respect to the value at the target, which is three times that predicted for the LFS divertor $(0.3)$. The significance of this result is deferred to the discussion in section 7.1.

\subsection{Effect of ELMs on the detached divertor plasma}

With the instantaneous input power to the divertor during the ELMs being of $\mathcal{O}(100) \mathrm{MW}$ (see section 2.5), it is impossible for the SOL plasma to buffer this energy by radiation and collisional processes, most of which reaches the targets. The imaging data reveals, however, that the ELM filaments are ejected into the far SOL, interacting with the targets away from the strike points. Their influence on the longer-term evolution of the inter-ELM detachment is hence predominantly through indirect effects.

Information on how the ionisation front moves during an ELM event can be obtained by comparing the NII emission for adjacent frames with and without ELMs during the exposures. Such a comparison is shown for the $\mathrm{N}$ II emissivity in figure 5 . It should be noted that the ELM crash duration of $\lesssim 1 \mathrm{~ms}$ is much less than the exposure time, so the images with ELMs are dominated by the integrated intensity from the inter-ELM periods. We can avail of the fact that the emissivity during the inter-ELM periods changes slowly with respect to the frame rate to obtain the emission due to the ELMs alone. For the frames with ELMs (right) we have subtracted the emissivity from the nearest (in time) ELM-free frame (left). By doing this, it can be seen that the ELMs result in ionisation of $\mathrm{N}_{2}$ in the far SOL, primarily close to the LFS target ${ }^{13}$. The efficacy of the subtraction implies that, on the timescale of the camera exposures, the ELMs have little effect on the emmisivity distribution over the rest of the SOL/divertor plasma.

The effect of the ELMs on the front locations is shown in figure 4 by the curves showing data from frames encompassing ELMs ((c), (d)-cyan). While the ELMs cause the peak $\mathrm{N}$ II emissivity (which increases strongly during the ELMs) to move close to the target, the peak location of the $\mathrm{D}_{\gamma} / \mathrm{D}_{\alpha}$ ratio hardly changes between the ELM/ELM-free frames. This can be understoood from the fact that the $\mathrm{D}_{\gamma}$ intensity increases at low temperature in a recombining plasma. During the ELMs, the hot plasma $\left(T_{e} \sim 400 \mathrm{eV}\right)$ ejected from the pedestal by the ELMs is strongly ionising, contributing little to the $\mathrm{D}_{\gamma}$ intensity. The measured $\mathrm{D}_{\gamma}$ intensity is hence primarily due to emission from the inter-ELM periods, with comparitively little contribution from the brief periods during the ELMs.

After the ELM crash (duration $\tau_{\text {ELM }} \lesssim 1 \mathrm{~ms}$ ), the ionisation balance of the SOL is evidently restored relatively quickly to that prevailing during the inter-ELM periods.

13 This emission is likely to be toroidally assymetric and hence the results of the inversions should be considered with caution. 

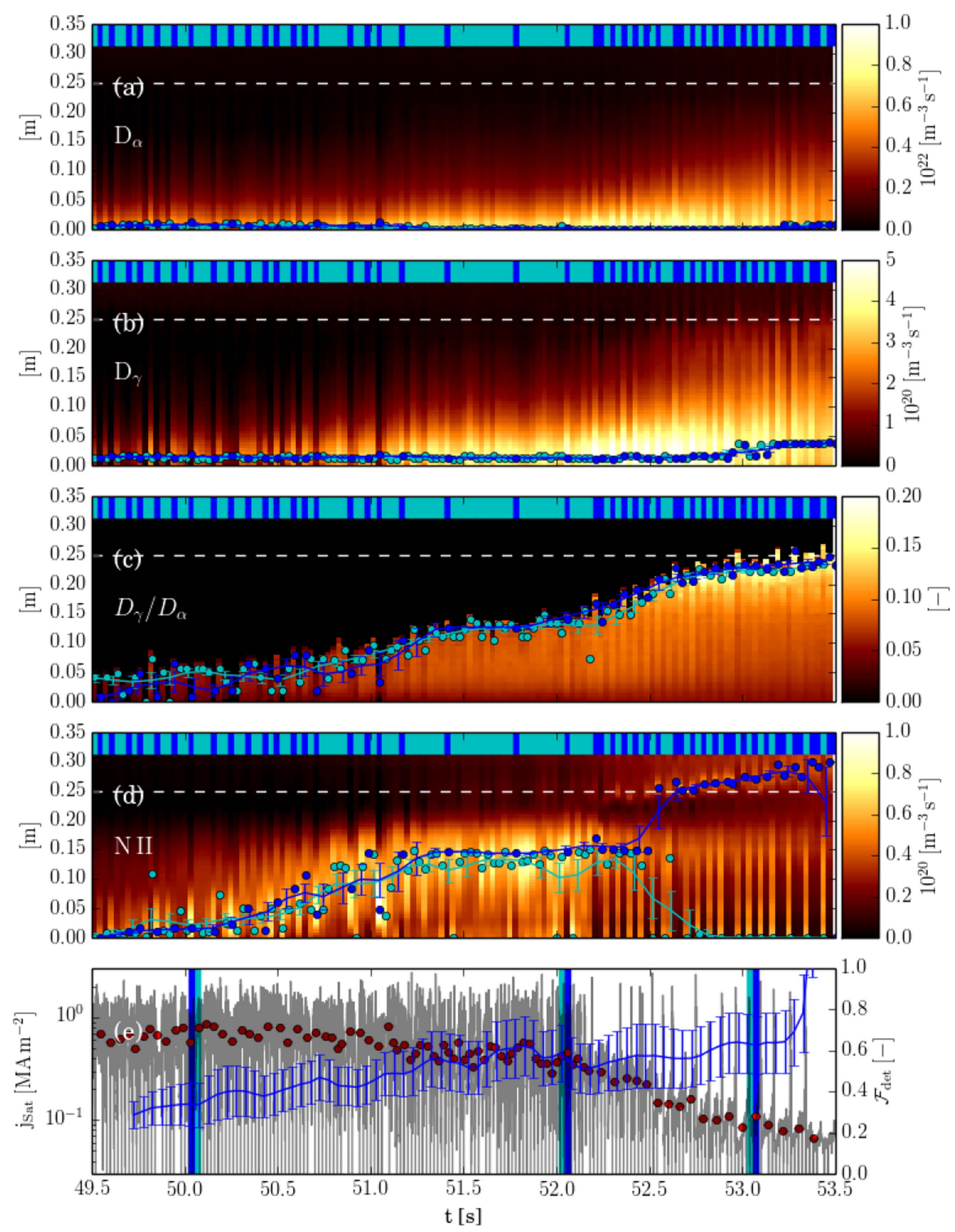

Figure 4. The evolution of line emissivity and emissivity ratio profiles along the flux surface through the S18C target Langmuir probe (shown in figure 5) during pulse \#89241 for: $\mathrm{D}_{\alpha}$ (a) and $\mathrm{D}_{\gamma}$ (b) intensities, the emissivity ratio $\mathrm{D}_{\gamma} / \mathrm{D}_{\alpha}$ (c) and $\mathrm{N}$ II (500.4 nm) intensity (d), together with the $j_{\text {sat }}$ signal through the same probe (grey) ((e), LH axis) (inter-ELM averaged values shown in red). The combined detachment control parameter $\mathcal{F}_{\text {det }}$ defined in section 1.2, calculated assuming $f_{I} \propto I_{\mathrm{NII}} / n_{u}$, is also shown (blue) ((e), RH axis). The locations of the profile maxima for frames with(without) ELMs are shown in (a)-(d) by the cyan(blue) lines respectively. Whether the frames encompass ELMs or are ELM-free is indicated by the upper, cyan/blue markers respectively. The horizontal (dashed-white) lines show the location at the same $z$ coordinate as the $\mathrm{x}$-point. The times of the frames shown in figures 3 and 5 are shown by the vertical blue/cyan bars in (e).

Hence, the ELMs only influence the detachment evolution indirectly by altering the net power input to the SOL and the radiation losses during the inter-ELM periods. These effects are quantified and discussed further in section 3.4.

\subsection{ELM energy losses}

Here, we quantify the ELM losses, both in terms of the instantaneous energy loss $\Delta W_{\mathrm{ELM}}$ and the time averaged
ELM-loss power $\left\langle P_{\text {ELM }}\right\rangle$ due to the repetitive ELMs. The former is compared to the energy deposited on the divertor target and the that radiated immediately after the ELMs. It is found by this crude form of energy balance that the SOL plasma must be cooled by the ELM sputtered impurities, leading to a brief period of ELM-induced detachment. The time-averaged ELM-loss power is required for calculation of the power crossing the separatrix between the ELMs, which is one of the control parameters for divertor detachment. 

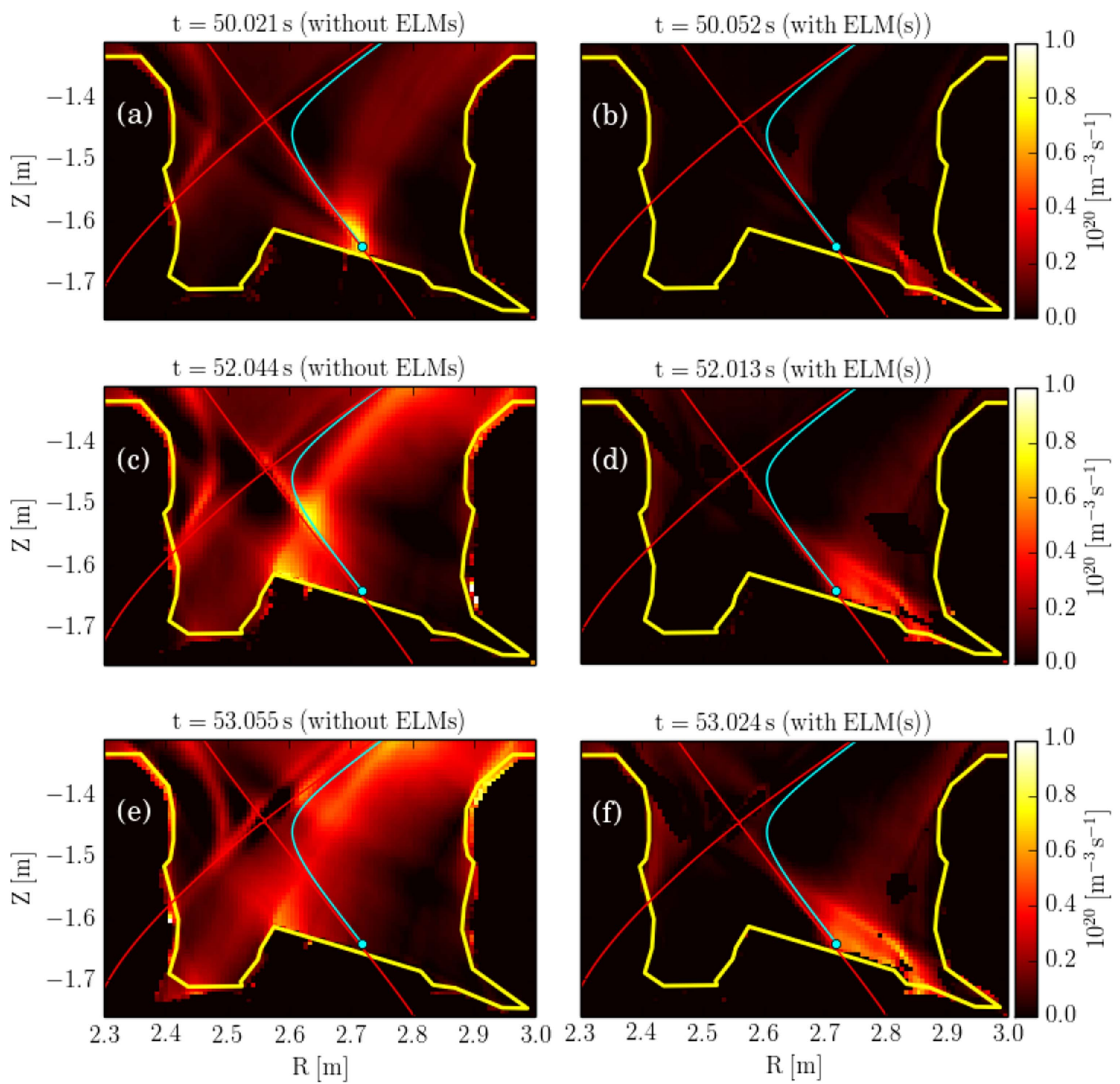

Figure 5. Distributions of N II (500.4 nm) line emissivity in pulse \#89241, comparing ELM-free frames (left) with frames encompassing ELMs (right). Note that for the frames with ELMs (right) the emission from the ELM-free frames (left) has been subtracted. Three cases are shown for times (indicated in figure 4(e) (no-ELM (blue), with-ELMs (subtracted) (cyan)) during the attached (a), (b), early-detached (c), (d) and late-detached (e), (f) phases.

Through their influence on the SOL input power and through their effect on the total radiation by sputtering impurities, the ELMs can thereby indirectly affect the inter-ELM detachment evolution.

The energy lost from the confined plasma during each ELM $\Delta W_{\text {ELM }}$ can be determined from the decrease in stored energy $W_{\text {MHD }}$ calculated from fast, $(\Delta t=0.5 \mathrm{~ms})$ equilibrium reconstructions (EHTR) using the EFIT code [27]. These losses are shown in figure 6(b), along with time-averaged values calculated using a sliding Gaussian window of duration $\tau_{\mathrm{sm}}$ of $0.25 \mathrm{~s}$, to be $\Delta W_{\mathrm{ELM}} \sim 50-150 \mathrm{~kJ}$, which correspond to power losses $\bar{P}_{\text {ELM }} \sim 50-150 \mathrm{MW}$ averaged over the $\sim 1 \mathrm{~ms}$ duration of the ELMs. This is much larger than the power into the divertor region during the inter-ELM periods $\left(P_{\text {Div }}^{\mathrm{i}-\mathrm{ELM}} \lesssim 2 \mathrm{MW}\right)$, which has to be determined from power balance (see section 3.1 below). The algorithm used to determine the time-averaged (or integrated) quantities is described in appendix B.

A slight decrease in magnitude of the ELM losses $\Delta W_{\text {ELM }}$ with increasing seeding is roughly compensated by an increase in the ELM frequency $f_{\text {ELM }}$ (figure 6(a)), as has been observed in earlier seeding experiments on JET with the carbon wall reported in [7]. This resulting in an almost constant time-averaged ELM loss power $\left\langle P_{\text {ELM }}\right\rangle \equiv$ $f_{\text {ELM }} \Delta W_{\text {ELM }} \sim 3 \mathrm{MW}$ (see figure 8 (a). This, together with the rather constant inter-ELM radiated power $P_{\mathrm{Rad}}^{\mathrm{i}-\mathrm{ELM}}$, results in the power to the divertor $P_{\mathrm{Div}}^{\mathrm{i}-\mathrm{ELM}}$ changing little during the inter-ELM periods until $52 \mathrm{~s}$. After this time, following the formation of the $\mathrm{x}$-point MARFE, the averaged ELM loss power $\left\langle P_{\mathrm{ELM}}\right\rangle$ does decrease, due to the step decrease in ELM frequency, which is not fully compensated by the increased $\Delta W_{\text {ELM. }}$. However, a concommittant increase in the 

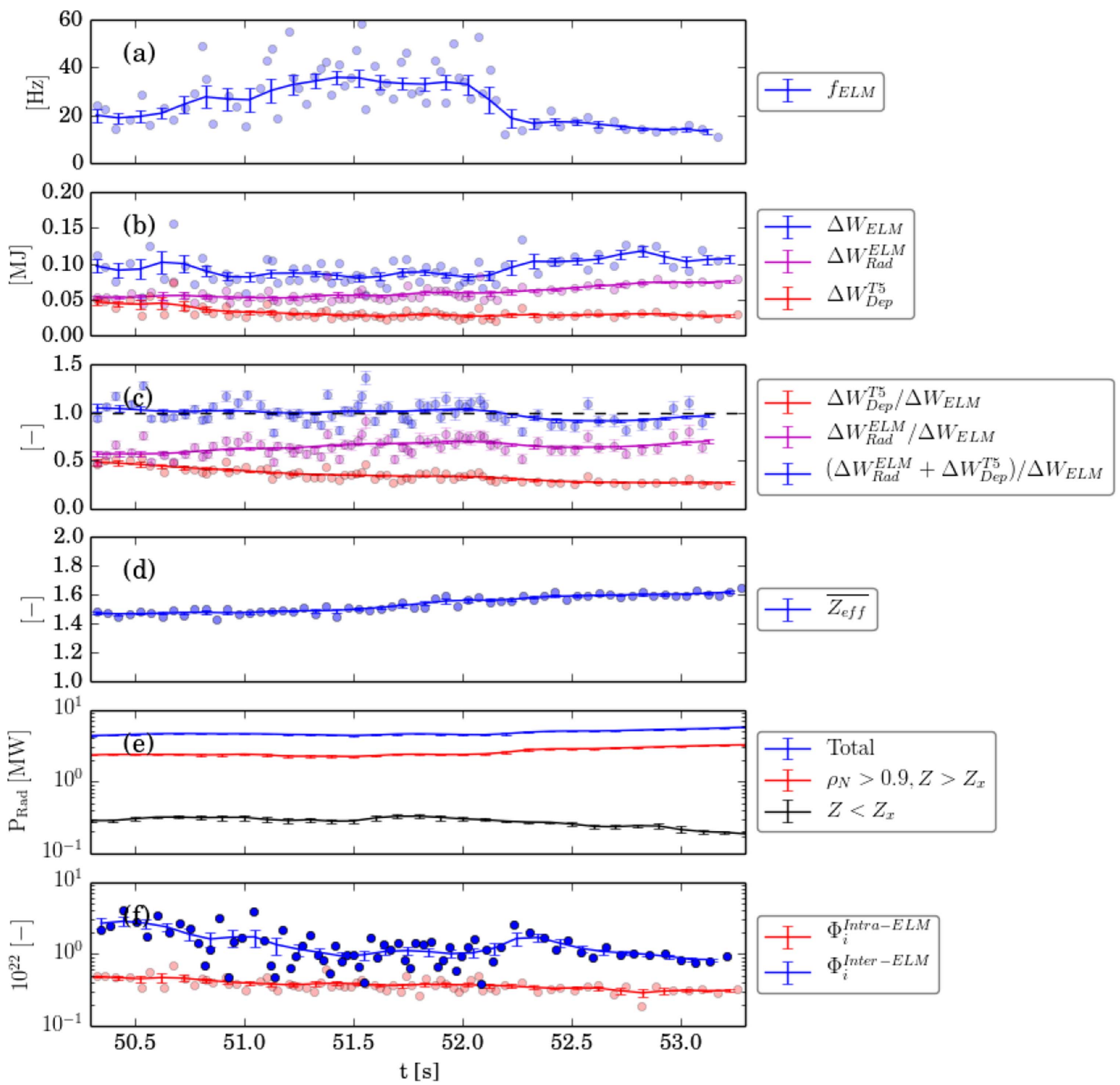

Figure 6. The evolution during pulse \#89241 of: (a) the ELM frequency $\left(\bullet f_{\mathrm{ELM}}=1 / \Delta t_{\mathrm{ELM}}\right.$, and $\left\langle f_{\mathrm{ELM}}\right\rangle=1 /\left\langle\Delta t_{\mathrm{ELM}}\right\rangle$, where $\Delta t_{\mathrm{ELM}}$ is the time between ELM peaks; (b) the energy losses $\Delta W_{\text {ELM }}$ (blue), total radiated energy $\Delta W_{\text {Rad }}^{\mathrm{ELM}}$ (magenta) and the deposited energy $\Delta W_{\text {Dep }}^{\mathrm{T5}}$ on T5 (red) during the intra-ELM periods; (c) ratios of $\Delta W_{\text {Dep }}^{\mathrm{T} 5} / \Delta W_{\mathrm{ELM}}$ (red), $\Delta W_{\mathrm{Rad}}^{\mathrm{ELM}} / \Delta W_{\mathrm{ELM}}$ (magenta) and $\left(\Delta W_{\mathrm{Rad}}^{\mathrm{ELM}}+\Delta W_{\text {Dep }}^{\mathrm{T5}}\right) / \Delta W_{\mathrm{ELM}}$ (blue); (d) the line-average, effective ion charge $\overline{Z_{\text {eff }}}$ from visible bremsstrahlung; (e) the inter-ELM radiated power $P_{\text {Rad }}^{\mathrm{i}-E L M}$ from various regions of the plasma (total-blue, mantle-red, divertor-black); and (f) the total ion fluences $\Phi_{i}^{\text {tot }}=\int \Gamma_{i}^{\text {tot }} \mathrm{d} t$ to both divertor targets during the intra-ELM (red) and inter-ELM (blue) periods. Time-averages of the data points over a sliding Gaussian window of duration $\tau_{\mathrm{sm}}$ of $0.25 \mathrm{~s}$ are represented by the solid lines.

radiated power $P_{\mathrm{Rad}}^{\mathrm{i}-\mathrm{ELM}}$ results in $P_{\mathrm{Div}}^{\mathrm{i} \text {-ELM }}$ remaining the total energy radiated $\Delta W_{\mathrm{Rad}}^{\mathrm{ELM}}$ during the ELMs, which is unchanged. generally higher than the energy deposited directly onto the

The energy deposited onto the sloping LFS target $\Delta W_{\text {Dep }}^{\mathrm{T} 5}$, measured using infra-red (IR) thermography (KL9A) [28, 29], is also shown in figure 6(b), where intra-ELM integrated values are $\lesssim 50 \mathrm{~kJ}$, i.e. typically $\lesssim 50 \%$ of the ELM energy losses. The time-dependent power deposition profile shown in figure 26(f) of section 6 shows that most of this energy is deposited during the initial ELM crash $\left(\tau_{\mathrm{ELM}} \lesssim 1 \mathrm{~ms}\right)$, resulting in peak target heat fluxes $q_{\mathrm{dep}}^{\mathrm{T5}} \sim \mathcal{O}\left(10^{\prime} \mathrm{s}\right) \mathrm{MW} \mathrm{m}^{-2}$. In comparison, during the inter-ELM periods the heat flux is much less $q_{\mathrm{dep}}^{\mathrm{T} 5} \sim \mathcal{O}(1) \mathrm{MW} \mathrm{m}^{-2}$, even when the divertor plasma is attached to the target. Also shown in figure 6(b) is target. However, as shown in section 6, this radiation peaks a few $m s$ after the time of the peak ELM heat flux ${ }^{14}$.

Ratios of the deposited and radiated energies during the intra-ELM periods to the total ELM energy loss are shown in figure 6(c): the ratio of deposited energy on T5 to the total ELM energy loss $\Delta W_{\text {Dep }}^{\mathrm{T5}} / \Delta W_{\mathrm{ELM}}$ is $\lesssim 50 \%$, decreasing

14 For the integration of the radiated power to calculate $\Delta W_{\mathrm{Rad}}^{\mathrm{ELM}}$, the ELM duration is defined as the time from the initial rise in the BeII intensity viewing the LFS target to the time at which it decays to $\leqslant 10 \%$ of its peak intensity, which is typically a few ms and longer than the duration of the ELM crash ( $\lesssim 1 \mathrm{~ms})$. 
slightly with increasing level of $\mathrm{N}_{2}$ seeding, while the ratio of radiated energy to loss energy $\Delta W_{\mathrm{Rad}}^{\mathrm{ELM}} / \Delta W_{\mathrm{ELM}}$ increases from $\sim 50 \%$ to $\sim 70 \%$. The issue of buffering of the ELM energy loss by the SOL plasma is discussed in more detail in section 7.4. Note that, because the IR measurements only cover part of T5 (stack C), the total deposited energy over the whole target is expected to be considerably higher.

As a crude form of energy accounting, the ratio of the sum of deposited and radiated energy to the ELM loss $\left(\Delta W_{\text {Rad }}^{\mathrm{ELM}}+\Delta W_{\text {Dep }}^{\mathrm{T5}}\right) / \Delta W_{\mathrm{ELM}}$ is found to be approximately unity, which is probably fortuitous as this measure does not include the ELM energy deposited on any other parts of the divertor target or vessel wall. Consequently, it is likely that the total energy losses during the ELMs considerably exceeds the ELM energy losses, resulting in a cooling of the plasma periphery immediately after each ELM. The fact that the peak radiation loss occurs a few ms after the peak ELM heat flux further reinforces this conclusion, which is consistent with observation of a brief period of detachment after each ELM crash (see section 6).

In a similar study of the energy balance during type-I ELMs on ASDEX-U by means of tomographic reconstructions of fast bolometer measurements [30], it was found that up to $40 \%$ of the ELM energy loss could be accounted for by radiation, mainly from the HFS of the divertor. When comparing the ELM energy loss with the sum of radiated and deposited power, overall power balance of the divertor plasma was found to be maintained both during and between ELMs. Note that fullfillment of overall energy balance in our analysis would require measurement of the deposited energy over all of the PFCs and also the change in thermal energy of the plasma over the intra-ELM periods to be taken into account.

\subsection{Total intra- and inter-ELM ion fluences}

By integrating the target ion fluxes both during and between the ELMs, it is possible to calculate the intra- and inter-ELM ion fluences to the targets. It is found that the ion fluence to the targets between the ELMs often dominates that expelled by the ELMs. A substantial fraction of this is probably due to inter-ELM filaments emitted from the pedestal into the SOL. As discussed in section 6 below, the power density at the targets associated with these filaments can reach $\mathcal{O}(10) \mathrm{MW} \mathrm{m}^{-2}$ under attached conditions.

Using the ion flux data $\Gamma_{i}^{\text {tot }}$, time-integrated ion fluences $\Phi_{i}^{\text {tot }}=\int \Gamma_{i}^{\text {tot }} \mathrm{d} t$ to both divertor targets during the intra-ELM and inter-ELM periods are calculated, as shown for pulse \#89241 in figure 6(f). Perhaps surprisingly, the ion fluences during the inter-ELM periods are up to an order of magnitude larger than the particles expelled by the ELMs, although as the level of $\mathrm{N}_{2}$ seeding increases, the inter-ELM fluences decrease by a factor $\sim 3$. As expected for a constant particle flux across the separatrix, as the ELM frequency increases the inter-ELM fluences decrease proportionally to the inter-ELM periods, except after $52 \mathrm{~s}$ when this inverse dependence is broken. It is clear that there is a substantial particle efflux across the separatrix between the ELMs due to a combination of diffusive and filamentary transport, which is dominant in determining the particle balance. Note that the measured ion flux at the target can be considerably higher than the particle flux crossing the separatrix due to recycling in the divertor plasma, the recycling factor potentially becoming very large in detached conditions.

\subsection{Target profiles during ELM cycle}

By conditionally averaging the target ion flux data $\Gamma_{i}^{\text {tot }}$, it is possible to study in detail the interaction of the ELMs with the targets at different stages of the ELM-cycle. It is found that, even without impurity seeding, a brief period of detachment occurs after each ELM, although the degree of post-ELM detachment deepens with increased seeding. The ELMs interact with the targets primarily in the far SOL but cause little increase in the peak ion saturation current at the strike points.

During the ELM crashes, the total ion flux $\Gamma_{i}^{\text {tot }}$, shown in figures 1 (e) and (f), increases strongly, by up to a factor $\sim 5$, from which one might conclude that the SOL plasma strongly re-attaches to both targets, however, detailed investigation reveals more complex behaviour. Profiles of the ion-saturation current $j_{\text {sat }}$ at various stages of the ELM cycle are shown in figure 7. During the ELM crash (a) and (b), the target $j_{\text {sat }}$ profiles develop 'shoulders', which extend outwards far from the strike points, with the SOL plasma incident on both vertical targets, particularly strongly on the HFS. Without seeding, during the ELM crash the peak $j_{\text {sat }}$ near the strike points is actually no higher than maximum values later during attached inter-ELM periods (see figures 7(f) and (h)). Most of the increases in $\Gamma_{i}^{\text {tot }}$ during the ELMs can hence be attributed to the ELM filament interaction with the targets far from the strike points.

Note that it has been shown in [32], by coherent averaging of measurements from the JET target LPs at the peak ion flux over many ELMs, that the electron temperature is sufficiently low $\left(T_{e} \sim 20-30 \mathrm{eV}\right)$ at the targets to obtain reliable measurements of the ion saturation current during ELMs. The low $T_{e}$ measured at the target is consistent with the free-streaming kinetic model, which predicts near-complete transfer of parallel energy from electrons to ions in order to maintain quasi-neutrality of the ELM filaments while they are transported to the divertor targets [33, 34].

Immediately after the ELMs (figures 7(c) and (d) $\left.\delta t_{\mathrm{ELM}}=2-8 \mathrm{~ms}\right), j_{\mathrm{sat}}$ is strongly reduced, with the DoD increasing with the level of NII emission from the divertor region. Already after $\delta t_{\mathrm{ELM}}>8 \mathrm{~ms}$ (e) and (f), with lower levels of $\mathrm{N}_{2}$ seeding the plasma reattaches at both strike points, whereas with higher levels of seeding the the detachment persists throughout the inter-ELM periods. The momentary detachment immediately after the ELMs is likely to be caused by combined effects of enhanced radiation losses from the SOL due to sputtered impurities (see figure 1(d)) as well as a concommittant decrease in the input power to the divertor $P_{\text {Div }}^{\text {tot }}$ after the ELMs caused by the decrease in pedestal pressure. The detailed behaviour of the $j_{\text {sat }}$ and deposited power $P_{\text {dep }}^{\mathrm{T} 5}$ profiles during the ELMs is described in section 6 . 

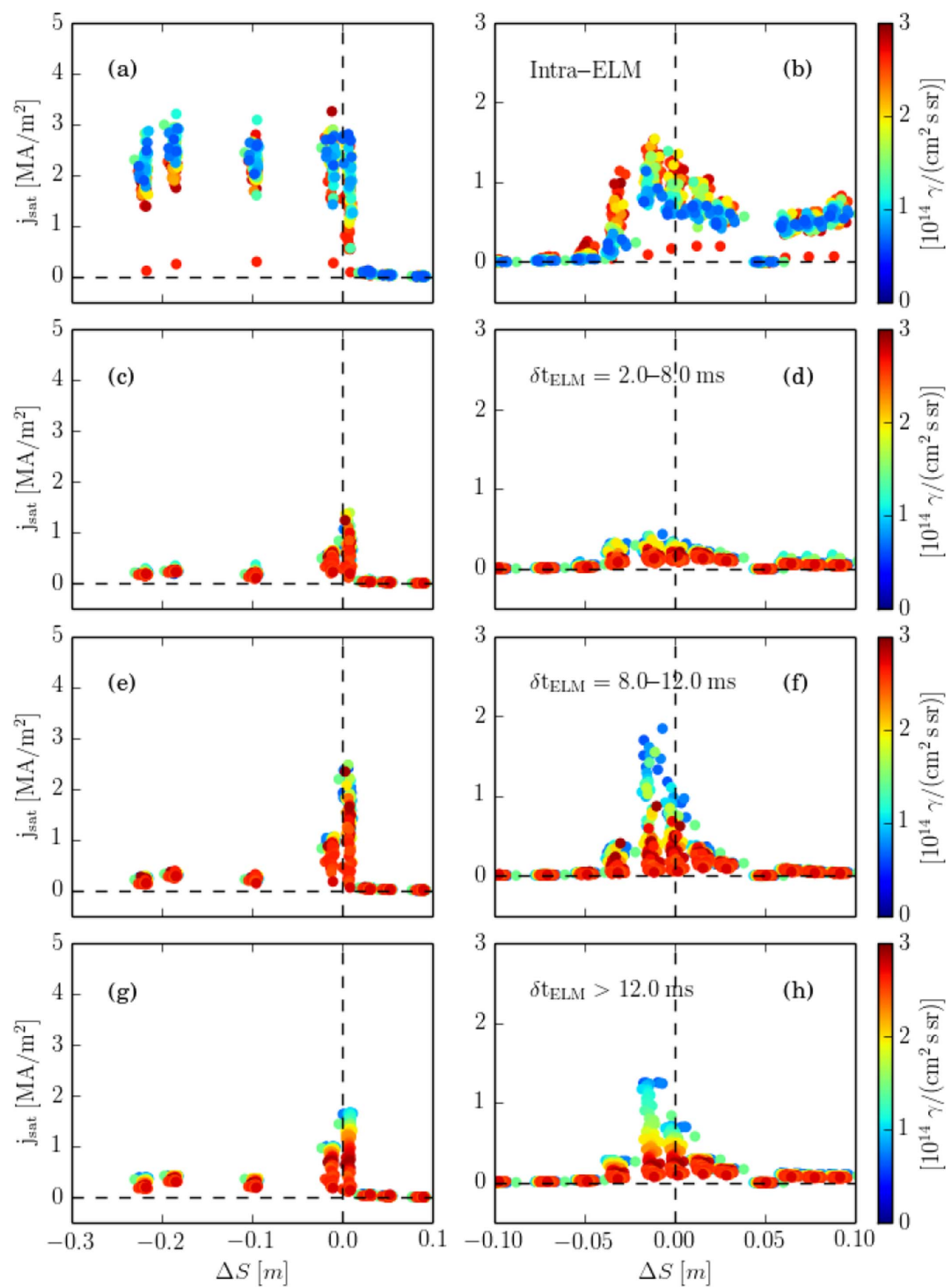

Figure 7. Profiles of the ion-saturation current during pulse \#89241 $j_{\mathrm{sat}}\left(\Delta S_{\mathrm{sp}}\right)$ measured the HFS (left) and LFS (right) using fixed Langmuir probe arrays, where $\Delta S_{\mathrm{sp}}$ is the probe separation along the target from relevant strike-point location $S_{\mathrm{sp}}$. The $j_{\mathrm{sat}}$ data is shown: during the ELM-crash periods (a), (b); immediately after the ELMs $\left(\delta t_{\mathrm{ELM}}=2-8 \mathrm{~ms}\right)(\mathrm{c}),(\mathrm{d})$; for $\delta t_{\mathrm{ELM}}=8-12 \mathrm{~ms}(\mathrm{e})$, (f) and for the remainder of the inter-ELM periods $\delta t_{\mathrm{ELM}}>12 \mathrm{~ms}(\mathrm{~g})$, (h). The intensity of NII $(500.0 \mathrm{~nm})$ line emission from the divertor regions, measured using a multichannel, visible range spectrometer (KS3) [31], is indicated by the color scale.

\section{Analysis of conditions promoting divertor detachment}

In this section, the experimental conditions promoting divertor detachment during the inter-ELM periods are determined in terms of appropriate control parameters. As discussed in section 1 and in the theoretical analysis of [14], these parameters are the upstream density $n_{u}$, here represented by the density at the mid-plane separatrix $n_{e, \text { sep }}$, the power crossing the separaterix into the SOL and hence into the divertor region $P_{\text {Div }}^{\text {tot }}$ and the concentration of impurity in the SOL plasma $f_{I}$. The latter is represented here by the intensity of NII $(500.0 \mathrm{~nm})$ line emission, which is approximately proportional to the impurity influx (see below).

The time-averaged, total power into the divertor region $P_{\mathrm{Div}}^{\text {tot }}$ can be calculated by subtracting the radiated power from the confined plasma $P_{\mathrm{Rad}}^{\mathrm{Pl}}$ from the thermal loss power $P_{1, \text { th }}$, i.e. the time-averaged, net power crossing the separatrix, which is determined from power balance. If, as here, we are interested in determining the conditions for detachment during the inter-ELM periods, we also need to subtract the time-averaged, ELM-loss power $\left\langle P_{\mathrm{ELM}}\right\rangle$ from $P_{\mathrm{Div}}^{\text {tot }}$ to determine the power input to the divertor region during these periods $P_{\mathrm{Div}}^{\mathrm{i}-\mathrm{ELM}}$. Details of this calculation are presented in section 3.1.

Seeding the plasma with extrinsic impurities is found to affect the characteristics of the H-mode pedestal, in particular the electron density $n_{e \text {,ped }}$ and temperature $T_{e \text {,ped }}$ at the pedestal top. The measurement of these parameters is described in section 3.2.1. These changes affect both the power radiated by the seeded impurities within the pedestal region and the characteristics of the ELMs, i.e. their amplitude and frequency. As discussed above, both of these can affect the net power into the divertor during the inter-ELM periods, which can in turn 
influence the propensity for detachment. The dependence of the DoD on the pedestal evolution and pedestal parameters are investigated in sections 3.2.2 and 3.2.3 respectively.

The observed changes in the pedestal characteristics $\left(T_{e \text {,ped }}\right.$ and $\left.n_{e \text {,ped }}\right)$ are not necessarily reflected in similar changes in the separatrix density $n_{e \text {,sep }}$ and temperature $T_{e \text {,sep }}$. This is because the latter are determined by the requirement for power balance between the power crossing the separatrix $P_{\text {Sep }}$ and the dominant, parallel heat flux along the field lines to the divertor targets $P_{\|, \mathrm{SOL}}$, rather than the physics which determines the pedestal characteristics ${ }^{15}$.

As explained in section 3.3, the separatrix power balance can be used to estimate the separatrix location, which is poorly defined by the high-time resolution EFIT equilibrium reconstructions. This method allows at least relative changes in the separatrix density $n_{e, \text { sep }}$ and temperature $T_{e, \text { sep }}$ to be determined, although to avail of this method it is still necessary to prescribe $T_{e \text {,sep }}$ at one time point. The results of this analysis are subsequently used in section 3.3 to investigate the dependence of the divertor detachment on the separatrix density and temperature.

Determination of the final third control parameter, the concentration of the seeded impurity $f_{I}$ would require detailed analysis of absolutely calibrated spectroscopic intensity measurements, e.g. as in figures $3(\mathrm{~g})-(\mathrm{i})$, using a suitable interpretive code, in particular requiring 2D distributions of $T_{e}$ and $n_{e}$ in the SOL. Instead, here, we assume that the nitrogen influx is approximately proportional to the NII $(500.0 \mathrm{~nm})$ line intensity and that the it's concentration is proportional to this influx. The dependence of the DoD on the level of impurity seeding is discussed in section 3.4.

\subsection{Determination of SOL power}

The power input to the divertor during the inter-ELM periods $P_{\text {Div }}^{\mathrm{i}-\mathrm{ELM}}$ is calculated, accounting both for the radiated power $P_{\text {Rad }}^{\mathrm{i}-\mathrm{ELM}}$ from the confined plasma and the time-averaged ELM loss power $\left\langle P_{\mathrm{ELM}}\right\rangle$. Surprisingly, this power varies little in pulse \#89241, in spite of the increasing seeding rate and consequential changes in ELM characteristics and total radiation. An uncertainty in the overall power balance in JET, potentially results in large fractional uncertainty in this important detachment control parameter.

On average, the total power crossing the separatrix and hence into the divertor, including the ELM power losses, $P_{\text {Div }}^{\text {tot }}$ is determined from $P_{\mathrm{Div}}^{\mathrm{tot}}=P_{\mathrm{l} \text {,th }}-P_{\mathrm{Rad}}^{\mathrm{Pl}}$, where $P_{\mathrm{Rad}}^{\mathrm{Pl}}$ is the radiated power from the confined plasma, here taken as the radiation from above the height of the x-point, $Z>Z_{\times}$. The thermal loss power $P_{1, \text { th }}$ is determined from power balance, i.e. $P_{1, \text { th }}=P_{\text {in }}-\left\langle\mathrm{d} W_{\mathrm{pl}} / \mathrm{d} t\right\rangle$, where $P_{\text {in }}$ is the total input power (from Ohmic plus NBI and ICRH auxilliary heating) and $\left\langle\mathrm{d} W_{\mathrm{pl}} / \mathrm{d} t\right\rangle$ represents a time-averaged value of the plasma

15 The power flowing along the SOL to the targets can be expressed in terms of the parallel and perpendicular heat flux as $P_{\mathrm{SOL}}=\int \nabla \cdot\left(q_{\|}+q_{\perp}\right) \mathrm{d} V$, where the integration is performed over the volume of the SOL between the divertor plates. Typically, $q_{\|}$is $\mathcal{O}\left(10^{3}\right)$ larger than $q_{\perp}$, so we can assume $P_{\|, \mathrm{SOL}}=\int \nabla \cdot q_{\|} \mathrm{d} V$, where $\nabla q_{\|}$can be expressed as $B / \mathrm{d} l \cdot\left[\kappa_{\|} / B \mathrm{~d} T / \mathrm{d} l\right]$, where $\kappa_{\|}$is the parallel conductivity and $l$ is the parallel length along the field lines [13]. stored energy ${ }^{16}$. The radiated power is measured by a multichannel resistive bolometer system (KB5) [23]. Estimates of the total radiation, including that from the divertor region, $P_{\text {Rad }}^{\text {tot }}$ (BOLO/TOPI) are obtained from an appropriately weighted sum of the line-integral measurements [35].

As can be seen from figure $1(\mathrm{~d}), P_{\mathrm{Rad}}^{\mathrm{tot}}$ increases strongly (factor $\sim 5$ ) during the ELMs, primarily due to radiation from the divertor and x-point region, resulting in a total radiated power fraction $f_{\mathrm{Rad}}^{\text {tot }} \gg 1$. An estimate of the radiation level from the main plasma $P_{\mathrm{Rad}}^{\mathrm{Pl}}(\mathrm{BOLO} / \mathrm{TOBH})$ is obtained from a sub-set of the horizontally viewing KB5 channels viewing only the upper half of the main plasma. This signal exhibits much smaller increases $(\sim 20 \%)$ during the ELMs than that of the total radiation. These increases in radiation are likely to be due to radiation from sputtered impurities in the divertor and SOL, hence, when calculating $P_{\mathrm{Div}}^{\mathrm{tot}}$, time-averaged values of $P_{\mathrm{Rad}}^{\mathrm{Pl}}$ determined during the inter-ELM periods only are used, i.e. $P_{\text {Div }}^{\text {tot }}=P_{1, \text { th }}-$ $\left\langle P_{\mathrm{Rad}}^{\mathrm{i}-\mathrm{ELM}}\right\rangle$, where the $\langle\cdot\rangle$ represents a time-average over a sliding Gaussian window of duration $\tau_{\mathrm{sm}}=0.25 \mathrm{~s}$ (see appendix B).

Although one might expect the loss power $P_{1, \text { th }}$ to be known accurately, a detailed study of the energy balance in JET-ILW presented in [9] reveals an overall deficit, with typically $25 \%$ of the total, calculated input energy unaccounted for by summing the measured total radiated energy $E_{\mathrm{Rad}}$ from bolometry and the deposited energy $E_{\mathrm{TC}}$ from tile calorimetry. Using this method, it was not possible to determine whether this is due to a deficit in the input power $P_{\text {in }}$ or some unmeasured loss, e.g. to an uninstrumented region of the vessel. Equally good regression fits could be obtained with all of the $25 \%$ deficit on the input energy $E_{\text {in }}$, with most due to a shortfall of the NBI power, or less on $E_{\text {in }}$ and a $40 \%$ deficit of deposited energy $E_{\mathrm{TC}}$, which is typically $30 \%-50 \%$ of the total loss. With a fractional power deficit $f_{\text {def }}$, the actual input power is related to the nominal power by $P_{\text {in }}=\left(1-f_{\text {def }}\right) P_{\text {in }}^{\text {nom }}$. The maximum range of loss power assuming $f_{\text {def }}=0 \%-25 \%$ is indicated by the negative uncertainties on $P_{1 \text {,th }}$ in figure 8 (a).

In order to determine the power into the divertor region during the inter-ELM periods $P_{\text {Div }}^{\mathrm{i}-\mathrm{ELM}}$, the ELM loss power also has to be subtracted from $P_{1, \text { th }}$ as well as the radiated power. Subtracting the instantaneous power $P_{\text {ELM }}$ from $P_{1, \text { th }}$ would result in negative values of $P_{\mathrm{Div}}^{\text {tot }}$ during the ELMs, while not affecting the inter-ELM values. Instead, the timeaveraged ELM-loss power $\left\langle P_{\mathrm{ELM}}\right\rangle$ has to be subtracted, resulting in the inter-ELM power to the divertor being given by: $P_{\mathrm{Div}}^{\mathrm{i}-\mathrm{ELM}}=P_{\mathrm{Div}}^{\mathrm{tot}}-\left\langle P_{\mathrm{ELM}}\right\rangle$. The calculation of $\left\langle P_{\mathrm{ELM}}\right\rangle$ is performed by calculating a weighted sum of $\Delta W_{\text {ELM }}$ over a sliding Gaussian window and then normalising integrated energy to the duration of the window (see appendix B). The resulting powers are shown in figure 8(a), where it can be seen that, following the reduction of the ELM frequency after $52 \mathrm{~s}$, the increase in inter-ELM radiation from the core plasma is compensated by a reduction in the time-averaged ELM loss power, resulting in an almost constant power into the SOL.

\footnotetext{
16 For the data in the SCAL/PLTH signal used here as $P_{1, \text { th }}$, the smooothing time constant used to calculate $\left\langle\mathrm{d} W_{\mathrm{pl}} / \mathrm{d} t\right\rangle$ is $\tau_{\mathrm{sm}}=1.0 \mathrm{~s}$.
} 

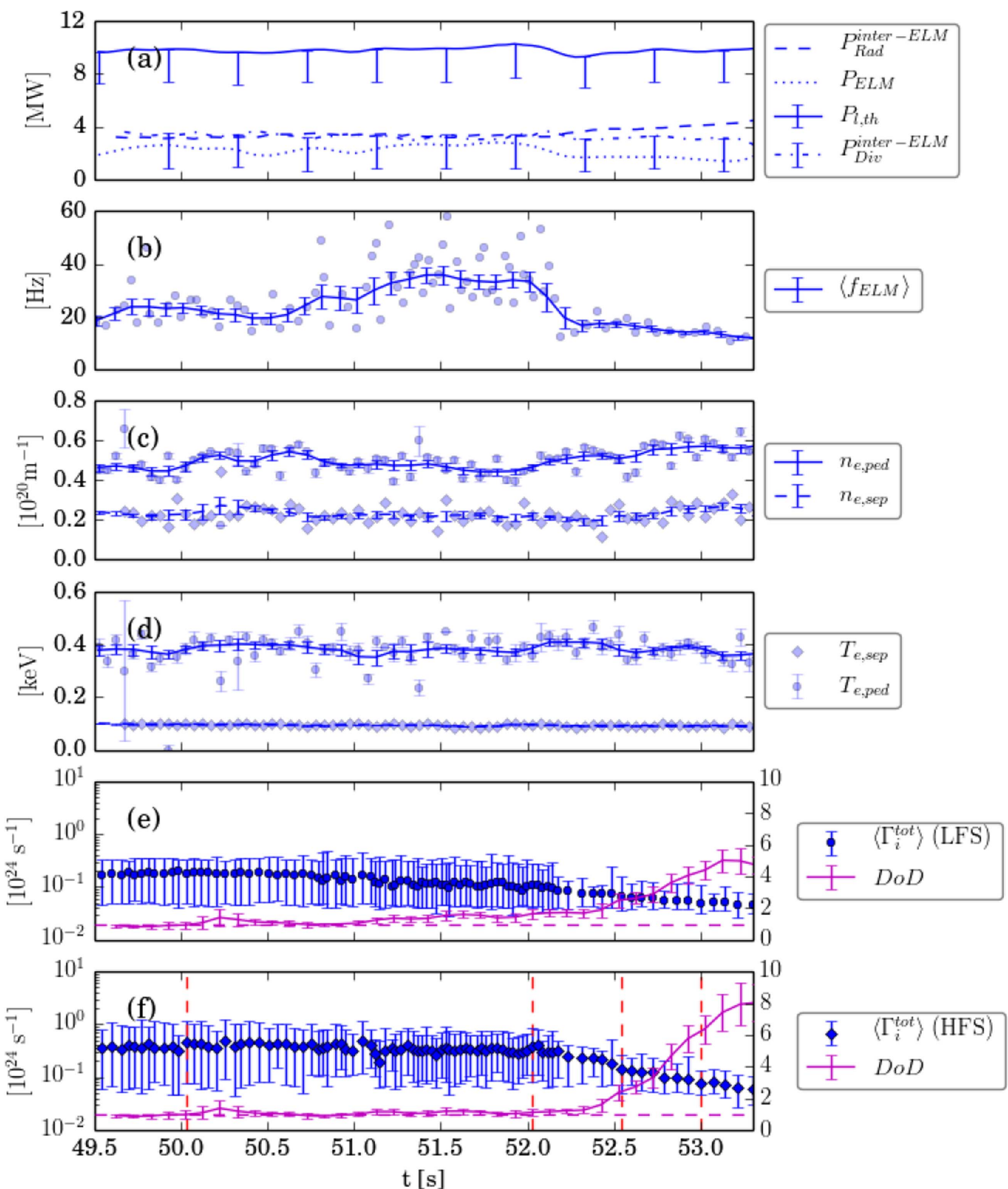

Figure 8. The evolution of relevant parameters of pulse \#89241 showing: (a) thermal loss power $P_{1, \mathrm{th}}$ (solid), ELM loss power $\left\langle P_{\mathrm{ELM}}\right\rangle$ (dotted), radiated power from confined plasma during inter-ELM periods $\left\langle P_{\mathrm{Rad}}^{\mathrm{i}-\mathrm{ELM}}\right\rangle$ (dashed), inter-ELM power into the divertor region $P_{\mathrm{Div}}^{\mathrm{i}-\mathrm{ELM}}$ (dotted-dashed); (b) ELM frequency $f_{\mathrm{ELM}}(\bullet)$ and $\left\langle f_{\mathrm{ELM}}\right\rangle$ (solid); (c) the pedestal $n_{e \text {,ped }}\left(\bullet\right.$ ) and separatrix $n_{e \text {,sep }}(\diamond)$ densities; (d) the pedestal $T_{e, \text { ped }}(\bullet)$ and separatrix $T_{e \text {,sep }}(\diamond)$ temperatures and (e), (f) the inter-ELM averaged total ion fluxes $\left\langle\Gamma_{i}^{\text {tot }}\right\rangle$ (error bars showing \{\}$_{\max }$ and \{\}$_{\min }$ fluctuations) to the LFS and HFS targets respectively.

Note that the possible deficit in the input power results in a very large fractional uncertainty in $P_{\mathrm{Div}}^{\mathrm{i}-\mathrm{ELM}}$, which could potentially lie between $\sim 1-4 \mathrm{MW}$. In section 3.4 an investigation of the overall, inter-ELM power balance of the divertor plasma reveals a discrepancy between the residual power $\left(P_{\mathrm{Res}}^{\mathrm{i}-\mathrm{ELM}}=P_{\mathrm{Div}}^{\mathrm{i} \text {-ELM }}-P_{\mathrm{Rad}}^{\mathrm{Div}}\right.$, where $P_{\mathrm{Rad}}^{\mathrm{Div}}$ is the radiation from below the x-point $Z \leqslant Z_{\times}$) with IR measurements of the deposited target power, which is consistent with a power deficit of similar magnitude to that found in [9].

\subsection{Dependence on pedestal characteristics}

In order to determine the separatrix density $n_{e, \text { sep }}$, one of the detachment control parameters, it is necessary to fit the $T_{e}$ and $n_{e}$ profiles measured by the HRTS system - a by-product of this is to determine the $\mathrm{H}$-mode pedestal parameters. Using this data, we have investigated how the target ion fluxes, which quantify the DoD, depend on these parameters. By means of coherent, ELM-cycle averaging, the dependence of the instantaneous detachment behaviour, as well as the level of fluctuations of the ion flux signal, on the inter-ELM pedestal evolution is investigated. Also, the deepest $\mathrm{DoD}$ is found to correlate with the lowest $T_{e}$ and highest $n_{e}$ at the pedetal top, probably due to enhanced radiation from the cooler, denser pedestal region, particularly in the presence of the x-point MARFE.

3.2.1. Determining the pedestal parameters. The H-mode pedestal parameters (height, width, position, offset and core 

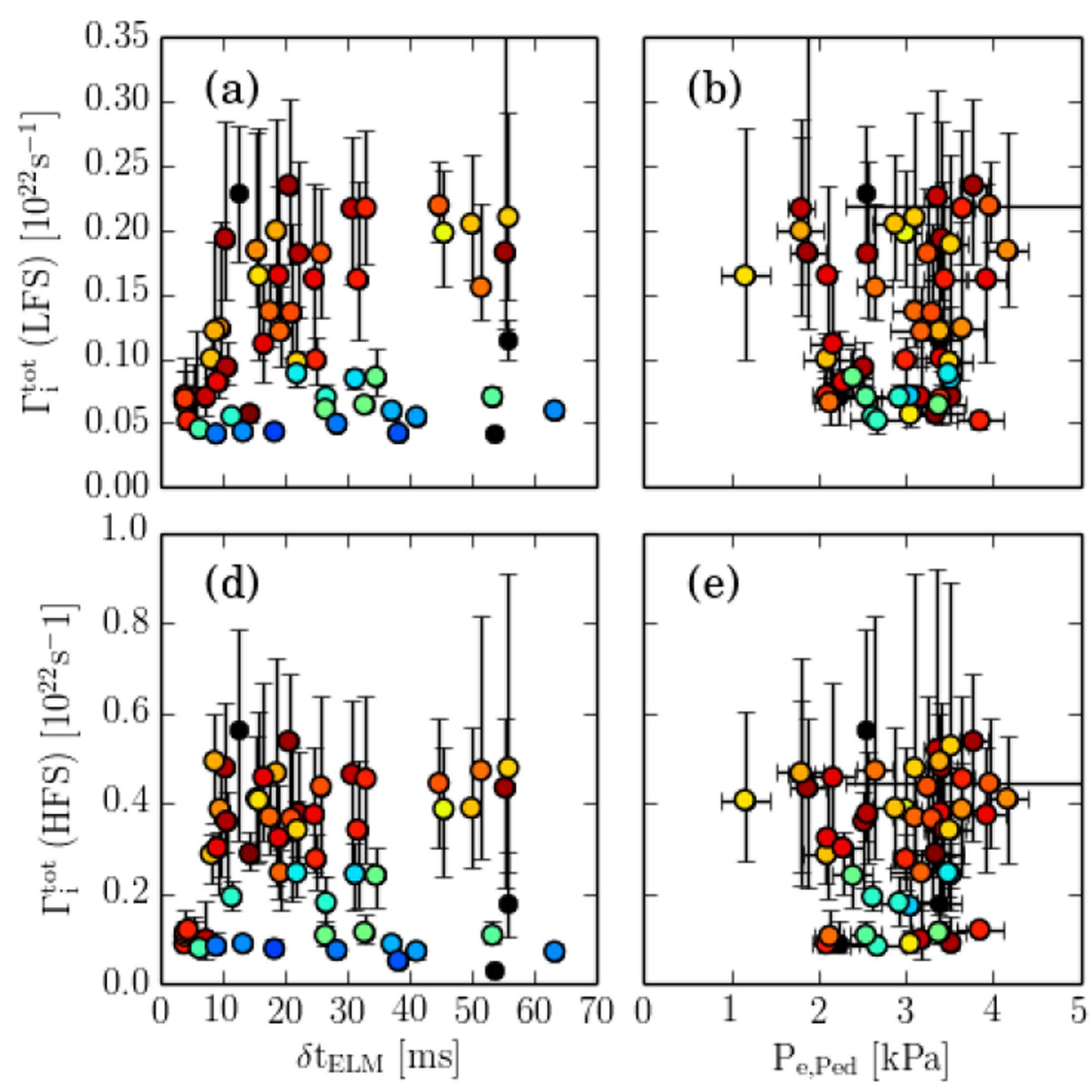
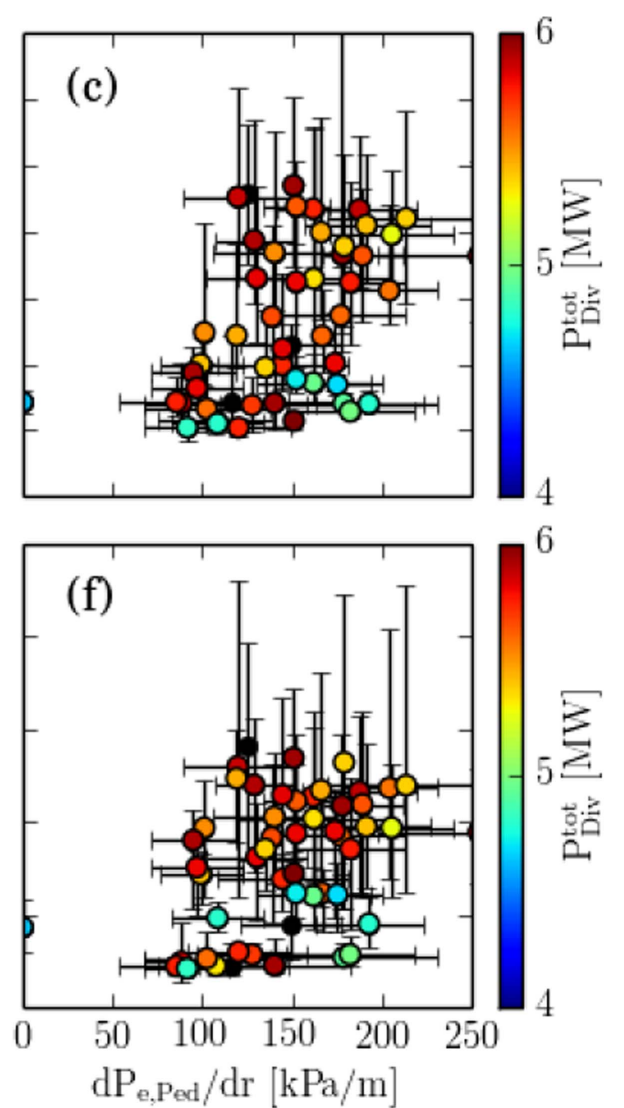

Figure 9. Average values of $\Gamma_{i}^{\text {tot }}$ (error bars represent \{\}$_{\max }$ and \{\}$_{\min }$ values) over $1 \mathrm{~ms}$ intervals during inter-ELM periods of pulse \#89241 (49-53.5 s) at the LFS (a), (b), (c) and HFS (d), (e), (f) targets as a function of time after the ELM crash $\delta t_{\mathrm{ELM}}$ (a), d), the pedestal top pressure $p_{e, \text { ped }}(\mathrm{b}),(\mathrm{e})$ and average pedestal pressure gradient $\left\langle\mathrm{d} p_{e \text {,ped }} / \mathrm{d} r\right\rangle(\mathrm{c})$, (f). The total input power to the divertor region $\left(Z \leqslant Z_{\times}\right) P_{\mathrm{Div}}^{\text {tot }}$ is represented by the color scale.

gradient of the $n_{e}, T_{e}$ and $p_{e}$ profiles) are obtained by performing mtanh fits [36] to HRTS measurements at the plasma periphery, which are available throughout the discharge at the $50 \mathrm{~ms}$ intervals of the laser pulses [8]. These times are then used to define the time periods for further analysis. The resulting data can be used to determine the dependence of the DoD, quantified here in terms of the reduction in $\Gamma_{i}^{\text {tot }}$, on the pedestal parameters. Average $\left\langle\Gamma_{i}^{\mathrm{tot}}\right\rangle$ and maximum $\left\{\delta \Gamma_{i}^{\mathrm{tot}}\right\}_{\max }$ and minimum $\left\{\delta \Gamma_{i}^{\mathrm{tot}}\right\}_{\text {min }}$ fluctuation amplitudes $\left(\delta \Gamma_{i}^{\mathrm{tot}}=\Gamma_{i}^{\mathrm{tot}}-\left\langle\Gamma_{i}^{\mathrm{tot}}\right\rangle\right)$ of the total ion fluxes measured at the LFS (KY4D/OTOF) and HFS (KY4D/ITOF) targets are determined during $1 \mathrm{~ms}$ intervals centred on the HRTS laser pulse times throughout the period of interest. These intervals are truncated (or the data omitted entirely) if they fall partly (or fully) within the intra-ELM period.

3.2.2. Dependence on inter-ELM pedestal evolution. The dependence of the total ion fluxes $\Gamma_{i}^{\text {tot }}$ to the LFS and HFS targets on: the time from the previous ELM $\delta t_{\mathrm{ELM}}$, the pedestal top pressure $p_{e \text {, ped }}$ and the average pedestal pressure gradient $\left\langle\mathrm{d} p_{e \text {, ped }} / \mathrm{d} r\right\rangle$ is shown in figure 9 for the pulse \#89241 with the $\mathrm{N}_{2}$ seeding ramp, for the period 49-52.5 s, which includes both attached and detached phases of the pulse. Immediately following the ELM crash $\Gamma_{i}^{\text {tot }}$ is reduced below $25 \%$ of the maximum inter-ELM values (corresponding to a DoD 3-4). Although during the ELM crash the loss power is far above the threshold required for re-attachment to both targets, the strong increase in $P_{\text {Rad }}^{\text {tot }}$ immediately following the ELMs (see figure 1(d)) from sputtered impurities results in cooling of the SOL plasma and its temporary detachment from both divertor targets.

At input powers $P_{\text {Div }}^{\text {tot }} \lesssim 5 \mathrm{MW}$ (color), the divertor plasma remains detached from both the LFS and HFS targets until the subsequent ELM, with $\Gamma_{i}^{\text {tot }}$ reduced to $\sim 25 \%$ of the maximum values observed during attached inter-ELM phases. From figures 9(a), (d) it can be seen that at higher levels of $P_{\text {Div }}^{\text {tot }}$ the divertor plasma re-attaches within $10 \mathrm{~ms}$ of the ELM crash. The high overall fluctuation level of $\Gamma_{i}^{\text {tot }}$ represented by the error bars $\left(\delta \Gamma_{i}^{\text {tot }} / \Gamma_{i}^{\text {tot }} \lesssim 30 \%\right)$ is also re-established within this timescale. These fluctuations are evidence of the emission of filaments from the pedestal or SOL during the inter-ELM periods [37], which might account for a substantial proportion of the total ion flux. As far as we are aware, however, no studies of the relative fluxes due to diffusive or filamentary transport have been made. The data presented in figure 9 and the temporal evolution of $\Gamma_{i}^{\text {tot }}$ (shown in figures 8(e), (f)) exhibit similar behaviour for the LFS and HFS divertors.

The dependence of $\Gamma_{i}^{\text {tot }}$ on the electron pressure at the pedestal top $p_{e \text {,ped }}$ is shown in figures $9(\mathrm{~b}),(\mathrm{e})$ and on the mean pedestal pressure gradient $\left\langle\mathrm{d} p_{e \text {, ped }} / \mathrm{d} r\right\rangle=p_{e \text {, ped }} / \Delta_{p_{e \text {, ped }}}$ (where $\Delta_{p_{e, \text { ped }}}$ is the pressure pedestal width determined from the mtanh fit) in figures 9(c), (f). It can be seen from this data that for these pulses a pedestal pressure $p_{e, \text { ped }} \gtrsim 1.5 \mathrm{kPa}$ and/or a pressure 

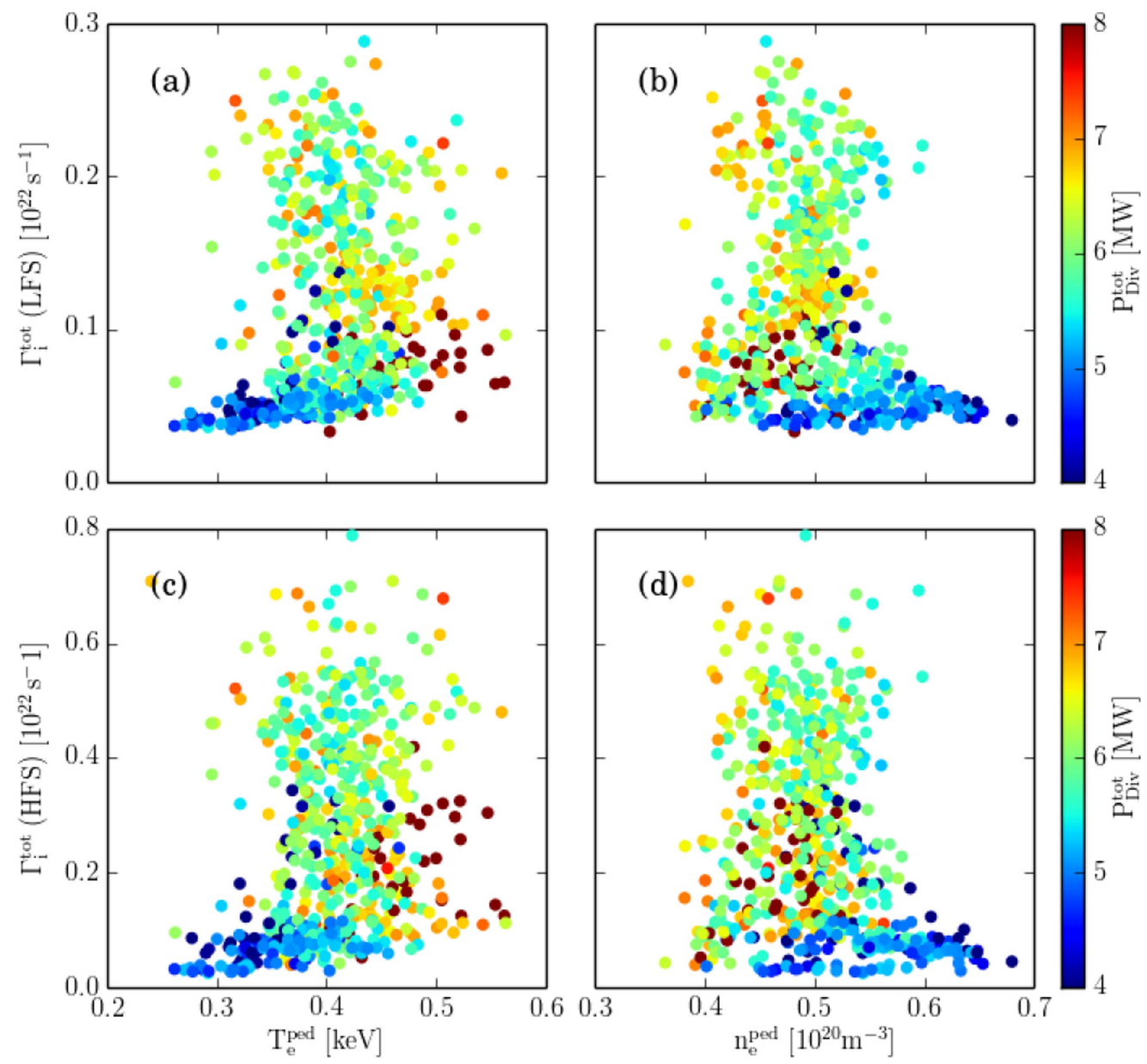

Figure 10. Average values of $\Gamma_{i}^{\text {tot }}$ over 1 ms intervals during inter-ELM periods at the LFS (a), (b) and HFS (c), (d) targets as a function of the electron temperature $T_{e \text {,ped }}$ (a), (c) and density $n_{e, \text { ped }}(\mathrm{b}),(\mathrm{d})$ at the pedestal top. The data is from the H-mode phases of the eight discharges analysed from this experiment (see section 2$)$. The total input power to the divertor region $\left(Z \leqslant Z_{\times}\right) P_{\mathrm{Div}}^{\text {tot }}$ is represented by the color scale.

gradient of $\left\langle\mathrm{d} p_{e \text {,ped }} / \mathrm{d} r\right\rangle \gtrsim 120 \mathrm{kPa} \mathrm{m}^{-1}$ is required to reestablish the full amplitude of inter-ELM filaments, which suggests that they might arise from some pressure driven instability in the pedestal. A recent study of pedestal evolution in JET-ILW pulses has shown the pressure gradient to be close to the stability boundary for infinite-n, kinetic-ballooning modes (KBMs) during the inter-ELM periods [38], so growth of such instabilities may be a possible origin for of the filaments.

3.2.3. Dependence on the pedestal parameters. Data from the same analysis can be used to determine the dependence of the DoD (quantified in terms of $\Gamma_{i}^{\text {tot }}$ ) on the pedestal temperature and density. Data from the eight discharges analysed from this experiment (see section 2) with various levels of $\mathrm{N}_{2}$ seeeding, including one unseeded reference discharge (\#89238) are shown in figure 10. From this it can be seen that the deepest detachment (characterised by a decrease of $\Gamma_{i}^{\text {tot }}$ to $\lesssim 20 \%$ of the maximum values) occurs when $P_{\text {Div }}^{\text {tot }} \lesssim 5 \mathrm{MW}$, with these conditions favoured by higher values of $n_{e \text {,ped }}$ and lower values of $T_{e \text {,ped }}$ which result in additional radiation from the seeded $\mathrm{N}_{2}$ impurity. These changes to the pedestal conditions caused by the seeding clearly affect the detachment behaviour by altering the input power to the divertor, both directly due to increased radiation from the confined plasma $P_{\mathrm{Rad}}^{\mathrm{Pl}}$ but also from changes to the ELM characteristics (amplitude and frequency), which in turn affect the time-averaged ELM power losses. These effects are further quantified in section 3.4 below.

\subsection{Dependence on separatrix characteristics}

The density $n_{e \text {,sep }}$ and temperature $T_{e \text {,sep }}$ at the separatrix are not necessarily governed by their values at the pedestal top, rather they are determined by a balance of cross-field and parallel particle and heat fluxes at the separatrix, respectively. Assuming parallel conduction dominates the heat flux in the SOL, the power crossing the separatrix can be used to determine the time-dependent $T_{e, \text { sep }}$. The coresponding density can then be determined from the HRTS data. Over the entire dataset from our experiments, no clear dependence of the $\mathrm{DoD}$ on $n_{e \text {, sep }}$ is revealed. This is a consquence of the data being from conditions with a wide range of seeded $\mathrm{N}_{2}$ impurity concentrations.

3.3.1. Determining separatrix parameters. Because the separatrix location is not determined to sufficient accuracy by the EFIT equilibrium reconstructions, the separatrix parameters cannot be determined directly from the HRTS measurements. Instead, an estimate of the separatrix radius at 

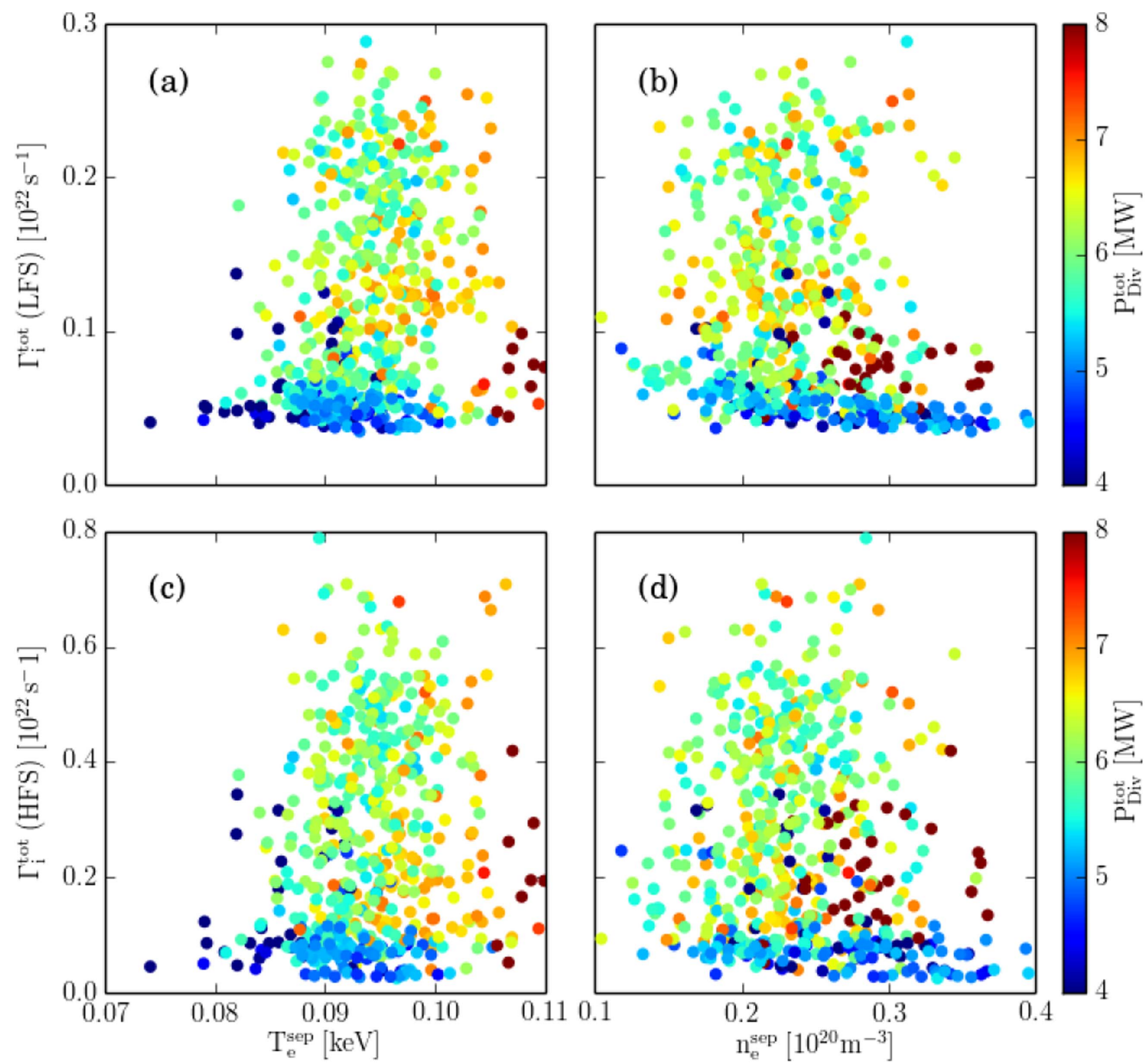

Figure 11. Average values of $\Gamma_{i}^{\text {tot }}$ over 1 ms intervals during inter-ELM periods at the LFS (a), (b) and HFS (c), (d) targets as a function of the electron temperature $T_{e \text {,sep }}$ (a), (c) and density $n_{e \text {,sep }}$ (b), (d) at the separatrix, which are determined from power balance assuming $P_{\|, \mathrm{SOL}} \propto T_{e, \text { sep }}^{7 / 2}$. The data is from the H-mode phases of the eight discharges analysed from this experiment (see section 2). The total input power to the divertor region $\left(Z \leqslant Z_{\times}\right) P_{\text {Div }}^{\text {tot }}$ is represented by the color scale.

the mid-plane $R_{\text {Sep }}$ (and hence a correction to the mapping from major radius $R$ to normalised radius $\rho_{N} \equiv r / a$ ) can be determined at each TS time point from a power balance argument using the known power into the SOL $P_{\text {SOL }}$ (here assumed equal to that into the divertor) and assuming a scaling of the form $P_{\|, \text {SOL }} \propto n_{e, \text { sep }}^{\alpha} T_{e \text {,sep }}^{\beta}$, where the proportionality constant can be determined by assuming a prescribed value of $T_{e \text {,sep }}$ at one time point. By this means, relative changes in the separatrix parameters can be determined if not absolute values.

In the conduction limited regime [39], the parallel electron heat flux is expected to scale as $q_{\|} \propto \nabla_{\|}\left(T_{e}\right) T_{e}^{5 / 2}$ (where $\nabla_{\|}=\mathrm{d} / \mathrm{d} l$ ). Furthermore, if we assume for simplicity that the parallel temperature gradient $\nabla_{\|}\left(T_{e}\right) \sim T_{e \text {, sep }} / L_{c}$ (where $L_{c}$ is the connection length from the mid-plane to the divertor target), the conducted power to the targets would be expected to scale as $P_{\|, \mathrm{SOL}} \propto T_{e, \text { sep }}^{7 / 2}$. Consequently, the separatrix temperature depends only weakly on the SOL input power, $T_{e, \text { sep }} \propto P_{\|, \mathrm{SOL}}^{2 / 7}$ and is therefore insensitive to small changes in the mapping of the profiles $\left(R_{\text {Sep }} \mapsto \rho_{N}\right)$.

Here, we assume power balance between the input power to the divertor during the inter-ELM periods and the conducted power in the SOL, i.e. that $P_{\mathrm{Div}}^{\mathrm{i}-\mathrm{ELM}}=C_{\mathrm{PB}} T_{e, \text { sep }}^{7 / 2}$, where $C_{\mathrm{PB}}$ is determined by fixing $T_{e \text {,sep }}$ at a reasonable value $(100 \mathrm{eV})$ at a particular time. Typically $C_{\mathrm{PB}} \sim 5$ with $T_{e}$ and $n_{e}$ in units of $\mathrm{keV}$ and $10^{20} \mathrm{~m}^{-3}$.

3.3.2. Dependence on the separatrix parameters. The results from this analysis for the eight pulses discussed previously are shown in figure 11. It can be seen that, whereas a clear dependence of the DoD on the pedestal parameters is observed (see figure 10), there is not such a clear dependence on the separatrix parameters. The lowest values of $\Gamma_{i}^{\text {tot }}$, correponding to detached conditions at lower $P_{\mathrm{Div}}^{\text {tot }}$, occur over the full range of $n_{e, \text { sep }}$, which varies by a factor of $\sim 2$. (Note that the range of $T_{e, \text { sep }}(\sim 20 \%)$ is much smaller than that of $n_{e \text {,sep }}$ because, as will be seen in section 3.4, the divertor input power $P_{\mathrm{Div}}^{\mathrm{i}-\mathrm{ELM}}$ is approximately constant.)

This lack of a clear dependence of detachment behaviour on $n_{e, \text { sep }}$ is at first sight contrary to expectations, however, this dataset is from plasmas with a range of $\mathrm{N}_{2}$ seeding rates and an unseeded reference pulse. With a higher level of seeding, more power will be radiated from the divertor plasma and, hence, detachment will occur at a lower value of upstream 

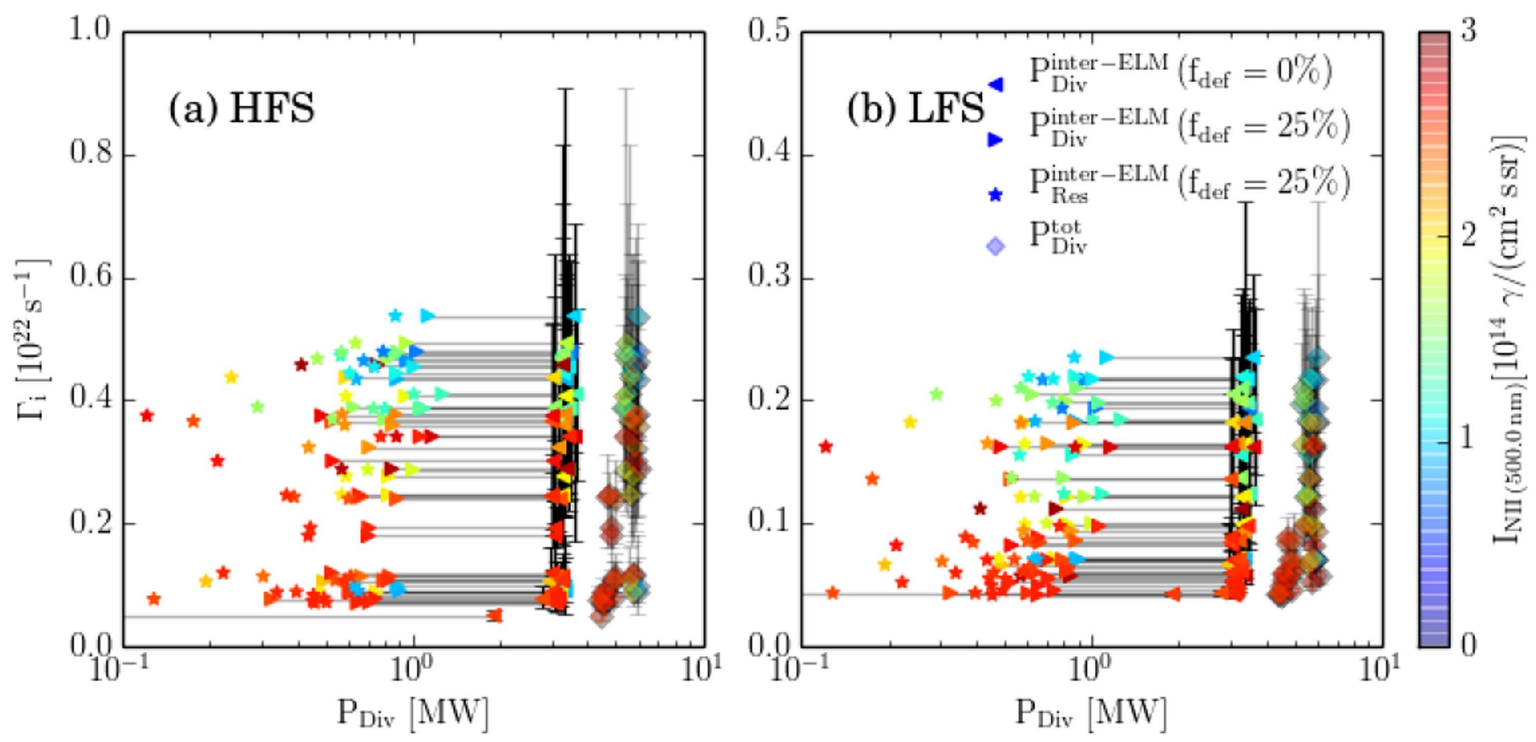

Figure 12. Average values of $\Gamma_{i}^{\text {tot }}$ (vertical error bars represent \{\}$_{\max }$ and \{\}$_{\min }$ fluctuations) during inter-ELM periods of pulse \#89241 at the HFS (a) and LFS (b) targets as a function of the total power into the divertor $\left(Z \leqslant Z_{\times}\right) P_{\text {Div }}^{\text {tot }}(\diamond)$, the inter-ELM divertor power $P_{\text {Div }}^{\mathrm{i}-\text { ELM }}(--4)$ and the residual power $P_{\text {Res }}^{\mathrm{i}-\text { ELM }}=P_{\text {Div }}^{\text {i-ELM }}-P_{\text {Rad }}^{\text {Div }}(\star)$. The sum of line-integrated NII (500.0nm) line intensities measured viewing the HFS (a) or LFS (b) divertor regions is represented by the color scale.

density than without seeding. This tendency is born out by the results presented in section 3.4 below.

\subsection{Dependence on divertor input power and impurity seeding}

The dependence of the DoD on the power input to the divertor and the level of seeded impurity is investigated. The possible shortfall in the overall power balance on JET implies a large uncertainty in this power. We attempt to quantify the input power deficit by performing a power balance analysis of the divertor SOL plasma. At the $10 \mathrm{MW}$ input power of these pulses, an approximate doubling of the $\mathrm{N}_{2}$ influx is sufficient to induce full detachment from both targets. The observation of intermittent detachment without seeding implies that the divertor input power is close to the threshold required to induce detachment in the unseeded pulses, which have somewhat lower separatrix density than those with seeding.

The dependence of the inter-ELM detachment, which is quantified in terms of the total ion flux $\Gamma_{i}^{\text {tot }}$ to the LFS and HFS targets, on the input power to the divertor is shown in figure 12 for pulse \#89241 with the $\mathrm{N}_{2}$ seeding ramp. Considering first the dependence on the total power $P_{\text {Div }}^{\text {tot }}$ (diamonds), it can be seen that, as the level of NII radiation increases, $P_{\text {Div }}^{\text {tot }}$ decreases slightly due to an increase in impurity radiation from within the main plasma $P_{\mathrm{Rad}}^{\mathrm{Pl}}$. At the lowest levels of $P_{\mathrm{Div}}^{\mathrm{tot}} \lesssim 5 \mathrm{MW}$ the SOL plasma is detached from the targets ${ }^{17}$.

\footnotetext{
17 The detachment behaviour clearly depends on the level impurity radiation from the SOL/divertor plasma, however, using either the $\mathrm{N}_{2}$ seeding rate $\Gamma_{\mathrm{N}_{2}}$ or fluence $\Phi_{\mathrm{N}_{2}}=\int \Gamma_{\mathrm{N}_{2}} \mathrm{~d} t$ to label the data points instead revealed no clear trend. This is because the level of impurity depends both on the direct influx from puffing and that from recycling, the relative contributions depending on the level of impurity retention by the PFCs. However, using instead the intensity of the NII $(500.0 \mathrm{~nm})$ spectral line measured by a multi-chord spectrometer (KSRA) viewing the HFS and LFS divertor regions as a measure of the level of seeded $\mathrm{N}_{2}$ does reveal clear trends.
}

Turning now to the divertor input power during the interELM periods, which is calculated by subtracting the time-averaged ELM-loss power, i.e. $P_{\text {Div }}^{\text {i-ELM }}=P_{\text {Div }}^{\text {tot }}-\left\langle P_{\text {ELM }}\right\rangle$, this amounts to $\sim 2.5-3.5 \mathrm{MW}$. This is comparable in magnitude to the maximum possible deficit in input power (discussed in section 3.1 ) of $2.5 \mathrm{MW}$ assuming $f_{\text {def }}=25 \%$. Hence, in figure 12 the possible range of $P_{\text {Div }}^{\text {i-ELM }}$ is indicated by the horizontal uncertainties between the markers (triangles). This highlights the difficulty posed by this power deficit [9] in determining the threshold power at which detachment occurs. As a first step in investigating the overall power balance, the residual power after subtracting the radiated power from the divertor from input power to the divertor, $P_{\mathrm{Res}}^{\mathrm{i}-\mathrm{ELM}}=P_{\mathrm{Div}}^{\mathrm{i} \text {-ELM }}-P_{\mathrm{Rad}}^{\mathrm{Div}}$ is also shown in figure 12 for the lowest possible values of $P_{\text {Div }}^{\mathrm{i}-\mathrm{ELM}}$ (stars). Clearly, if the power deficit is as high as $25 \%$, almost no residual power $(\lesssim 1 \mathrm{MW})$ would be measured at the divertor targets.

The power balance of the divertor during the inter-ELM periods is hence investigated in more detail in figure 13, which shows the deposited power measured on T5/C by the IR thermography $P_{\mathrm{dep}}^{\mathrm{T} 5}$ as a function of the portion of the residual power $f_{\mathrm{P}_{\mathrm{Res}}} \times P_{\mathrm{Res}}^{\mathrm{i} \text {-ELM }}$ incident on T5C. In the absence of measurements of the spatial distribution of the deposited power we make the assumption here that $f_{\mathrm{P}_{\mathrm{Res}}}=50 \%$. Firstly, considering conditions with a low seeding level with an attached divertor (figure 13(a)), approximate agreement between $P_{\mathrm{dep}}^{\mathrm{T} 5}$ and $P_{\text {Res }}^{\mathrm{i} \text {-ELM }}$ is found for an input power deficit $f_{\text {def }}=15 \%$, which is well below the maximum deficit of $25 \%$ found in [9].

As shown in figure 13(b), as the level of $\mathrm{N}_{2}$ seeding is increased, the approximate linear dependence of $P_{\mathrm{dep}}^{\mathrm{T} 5}$ on $P_{\text {Res }}^{\mathrm{i} \text {-ELM }}$ is maintained, except at the highest seeding level (which also corresponds to the one pulse \#89244 at higher input power). Note that, from the available data, it is not possible to aportion the power deficit between a shortfall in the input power and an as-yet unmeasured loss channel. 

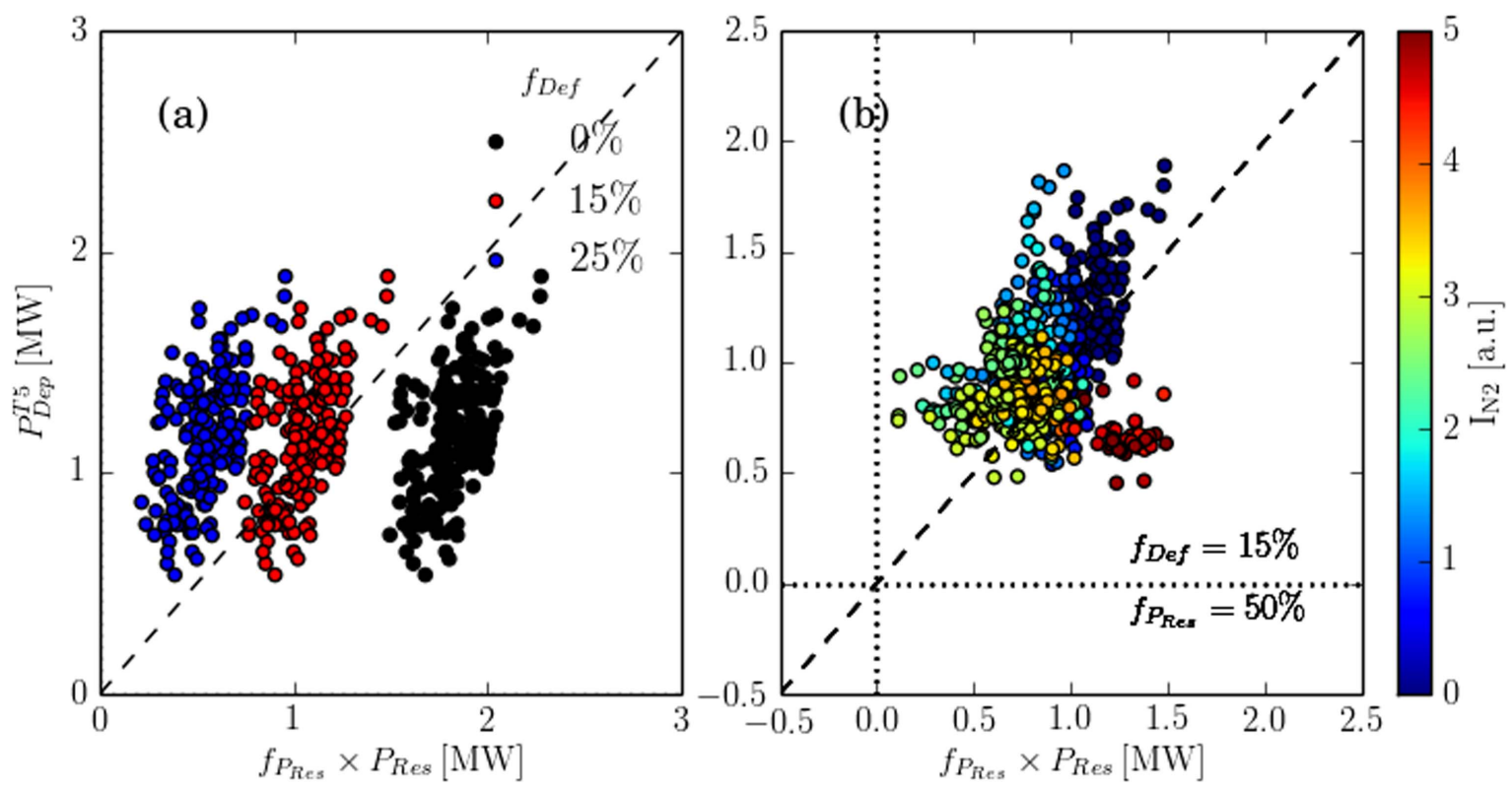

Figure 13. The measured power deposited on T5 $P_{\mathrm{dep}}^{\mathrm{T} 5}$ versus the residual power $P_{\mathrm{Res}}^{\mathrm{i}-\mathrm{ELM}}=P_{\mathrm{Div}}^{\mathrm{i} \text {-ELM }}-P_{\mathrm{Rad}}^{\mathrm{Div}}$ during inter-ELM periods: (a) assuming input power deficits of $f_{\text {def }}=0 \%, 15 \%$ and $25 \%$, selecting data with $\leqslant 20 \%$ of the maximum seeding level; and (b) for all seeding levels assuming $f_{\text {def }}=15 \%$ for all eight of the pulses analysed for this experiment. The fraction of $P_{\mathrm{Res}}^{\mathrm{i} \text {-ELM }}$ incident on T5/C $f_{\mathrm{P}_{\text {Res }}}$ is assumed here to be $50 \%$.

Henceforth, we assume an input power deficit $f_{\text {def }}$ of $15 \%$ in our subsequent analysis.

By combining data from all of the analysed pulses from this experiment (except the high power pulse \#89244), evidence for a power threshold for detachment is revealed in figure 14, which shows the dependence of the target ion fluxes on the uncorrected, total divertor input power $P_{\text {Div }}^{\text {tot }}(\diamond)$, including the ELM losses, and on corrected values of the input power to the divertor during the inter-ELM periods $P_{\text {Div }}^{\text {i-ELM }}(\bullet)$, calculated assuming $f_{\text {def }}$ of $15 \%$. The NII $(500.0 \mathrm{~nm})$ line intensity (color) measured viewing the divertor where the $\mathrm{N}_{2}$ is puffed, is approximately proportional to the influx and gives a measure of the seeded impurity fraction.

Perhaps counter-intuitively, the data points from conditions with little $\mathrm{N}_{2}$ seeding (blue) are at levels of $\Gamma_{i}^{\text {tot }}$ indicative of both attached and detached conditions, a phenomenon which is discussed in more detail in section 3.5 below. The inter-ELM power for these unseeded points $P_{\text {Div }}^{\mathrm{i}-\mathrm{ELM}} \sim 2-3 \mathrm{MW}$ is actually higher than those from seeded pulses because the radiation from the main plasma is less. The unseeded pulses are actually at lower Greenwald density fraction $f_{\mathrm{GW}}$ and hence lower separatrix density $n_{e \text {,sep }}$ than the seeded pulses. Consequently, the threshold power for detachment is expected to be somewhat higher in the unseeded than in the seeded pulses.

With $\mathrm{N}_{2}$ seeding, the divertor input power is slighly lower than without, i.e. $P_{\mathrm{Div}}^{\mathrm{i}-\mathrm{ELM}} \sim 1.5-2.5 \mathrm{MW}$ and, as expected, the DoD is observed to deepen as the level of $\mathrm{N}_{2}$ seeding increases. Clearly, the threshold divertor input power for the commencement of detachment lies close to $2 \mathrm{MW}$. In comparison to this, the radiation from the divertor region $P_{\mathrm{Rad}}^{\text {Div }}$ is substantially less, i.e. $\lesssim 0.5 \mathrm{MW}$ (see figure $8(\mathrm{e})$ ). Note, however, that due to the known discrepancy in the power balance, the potential uncertainty on the divertor input power $P_{\mathrm{Div}}^{\mathrm{i}-\mathrm{ELM}}$, as indicated by the horizontal error bars in figure 12, remains large in spite of our efforts to determine this from power balance.

\subsection{Dependence on Greenwald density fraction}

Investigation of the dependence of the detachment behaviour on the Greenwald fraction reveals that in seeded pulses at higher density, increased radiation from the core plasma, which is partially offset by reduced ELM power losses, leads to a greater propensity for detachment.

Increasing the $D_{2}$ fuelling by gas puffing increases the line-averaged density $\overline{n_{e}}$, here quantified in terms of the Greenwald density fraction $f_{\mathrm{GW}}$. Corresponding increases in the pedestal density $n_{e, \text { ped }}$ increase the radiation from the confined plasma $P_{\mathrm{Rad}}^{\mathrm{Pl}}$, thereby reducing the total power to the divertor $P_{\text {Div }}^{\text {tot }}$, and also affect the ELM characteristics. Typically, the fractional ELM energy losses characterised by $\Delta W_{\mathrm{ELM}} / W_{\mathrm{Ped}}$, are found to decrease with increasing $f_{\mathrm{GW}}$, e.g. as reported in [40]. Here, there is a modest decrease in $\left\langle P_{\mathrm{ELM}}\right\rangle$ with increasing seeding, so the decrease in $\Delta W_{\mathrm{ELM}}$ is only partially compensated by the higher ELM frequency.

Figure 15 shows the same data as in figure 14, except the color scale represents the difference of the normalised density $\left(f_{\mathrm{GW}}-1\right)$ to the Greenwald limit. It can be seen that at higher Greenwald density fraction $f_{\mathrm{GW}}$ the divertor plasma shows greater propensity to detach from the targets due to the increased radiation from the confined plasma and consequently decreased power into the divertor $P_{\text {Div }}^{\text {tot }}$. This dependence is partly because the line-averaged density, and hence $f_{\mathrm{GW}}$, increases together with the level $\mathrm{N}_{2}$ seeding but also because at a given level of seeding more power is radiated at higher density. 

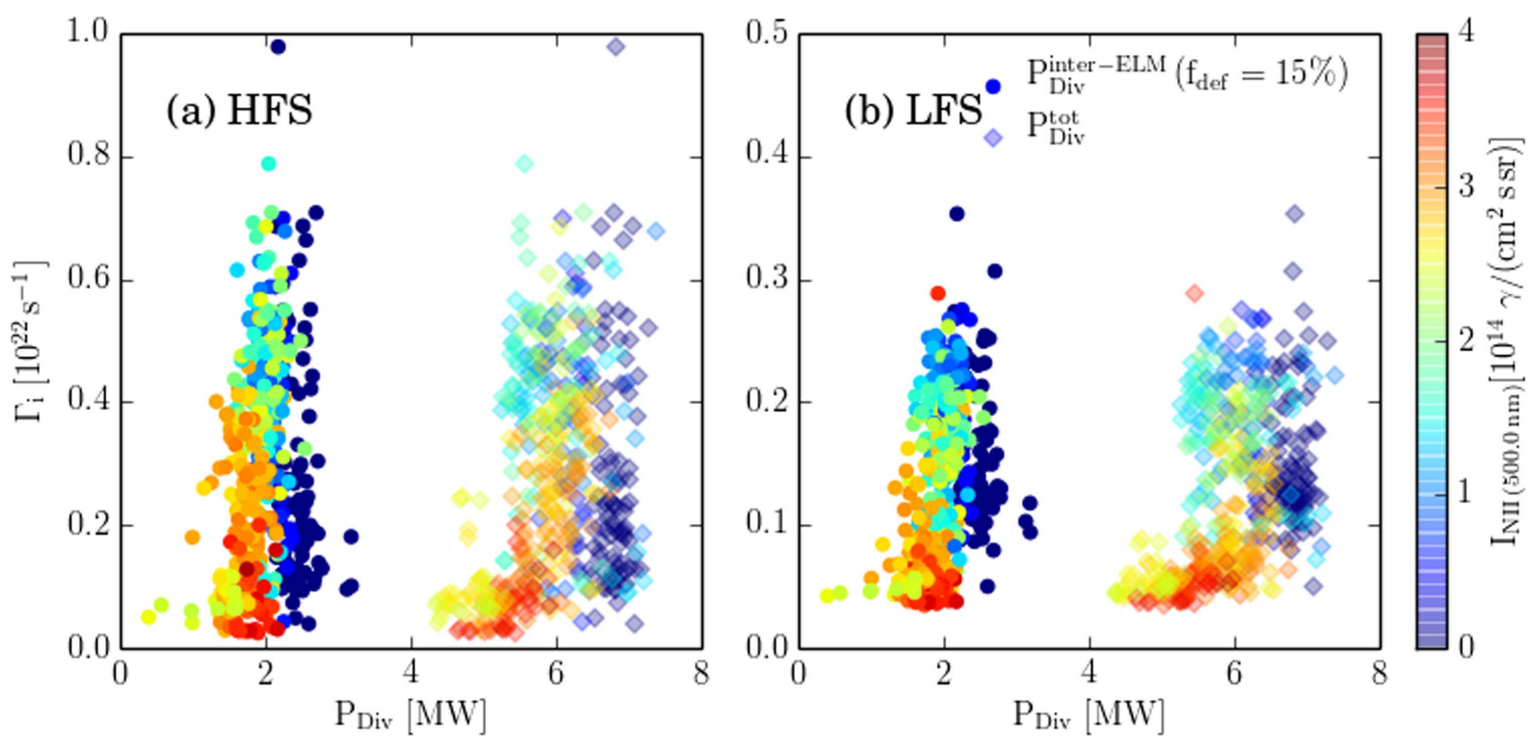

Figure 14. Average values of $\Gamma_{i}^{\text {tot }}$ during inter-ELM periods at the HFS (a) and LFS (b) targets as a function of the total input power to the divertor $P_{\mathrm{Div}}^{\text {tot }}(\diamond)$ and the corrected divertor input power during the inter-ELM periods $P_{\mathrm{Sep}}^{\mathrm{i}-\mathrm{ELM}}(\bullet)$ (assuming $f_{\text {def }}=15 \%$ ) for all of the pulses analysed from this experiment (except \#89244 with the NBI power ramp). The sum of line-integrated NII (500.0nm) line intensities measured viewing the HFS (a) or LFS (b) divertor regions is represented by the color scale.
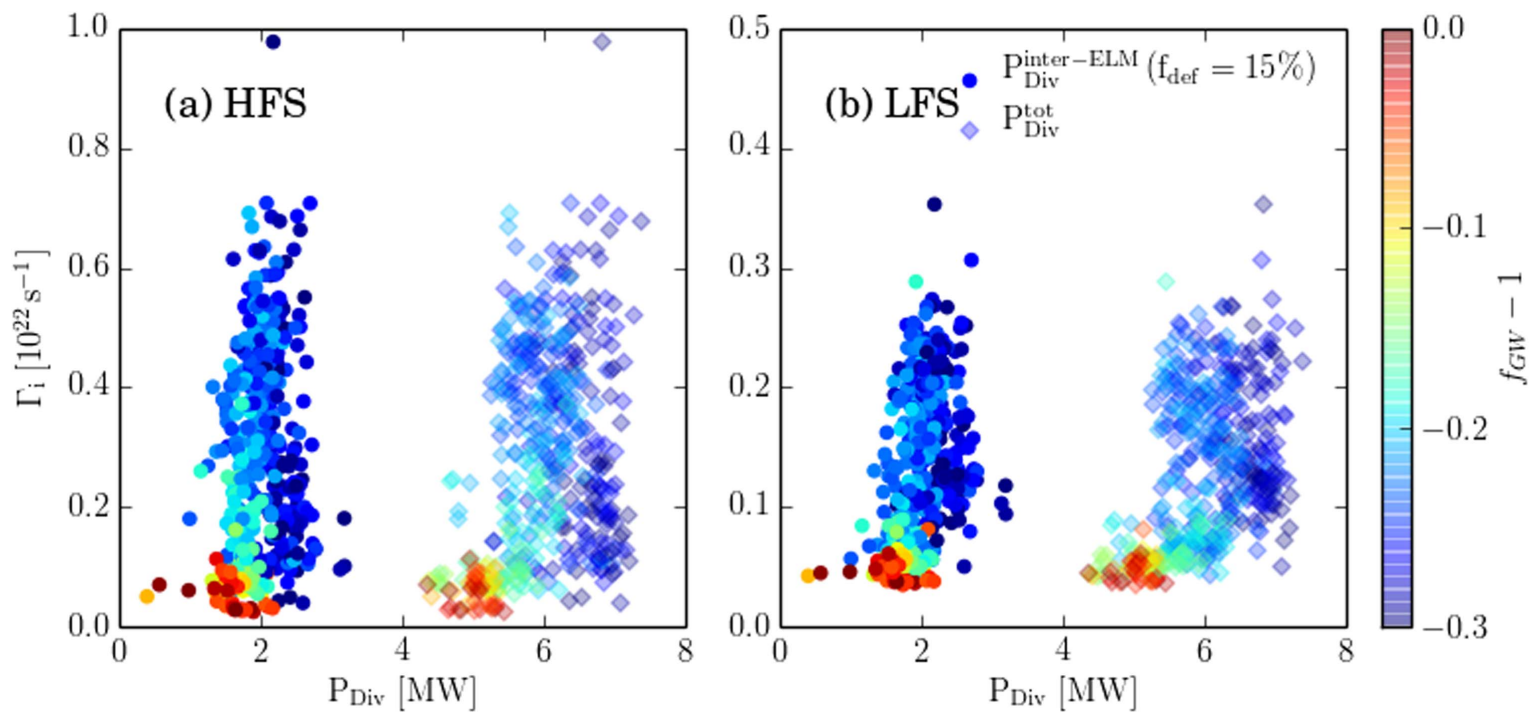

Figure 15. Average values of $\Gamma_{i}^{\text {tot }}$ during inter-ELM periods at the HFS (a) and LFS (b) targets as a function of the total power input to the divertor $P_{\mathrm{Div}}^{\text {tot }}(\diamond)$ and the divertor input power during the inter-ELM periods $P_{\mathrm{Div}}^{\mathrm{i} \text {-ELM }}(\bullet)\left(\right.$ corrected, assuming $\left.f_{\text {def }}=15 \%\right)$. The difference of the normalised density from the Greenwald limit $f_{\mathrm{GW}}-1$ is represented by the color scale.

There is less of a difference in the divertor power input during the inter-ELM periods $P_{\mathrm{Div}}^{\mathrm{i}-\mathrm{ELM}}$ between the seeded and unseeded pulses than for the total divertor power $P_{\text {Div }}^{\text {tot }}$ because the ELM power loss $\left\langle P_{\mathrm{ELM}}\right\rangle$ is somewhat lower with $\mathrm{N}_{2}$ seeding than without.

\section{Unstable divertor detachment at threshold SOL power}

Here, we investigate the unstable, oscillatory detachment behaviour observed in the unseeded pulses in more detail. Spectroscopic and bolometric data is consistent with an interpretation that the phenomenon may be due to a radiative instability occuring at near-threshold divertor input power, induced by impurities sputtered from the targets during the attached phase of the oscillations.

The observation of levels of $\Gamma_{i}^{\text {tot }}$ indicative of both attached and detached divertor conditions for the data from the unseeded pulse in figure 14 is explored in more detail in figure 16. During the $50 \mathrm{~ms}$ period shown between the two ELM events, indicated by the strong peaks in the BeII signals, there are oscillations of the ion fluxes $\Gamma_{i}^{\text {tot }}$ to both targets with a period of $\sim 10 \mathrm{~ms}$. The peaks in BeII signals are indicative of sputtered impurity influx from the strike points at both targets. Radiation from the sputtered impurities (W, Be and 

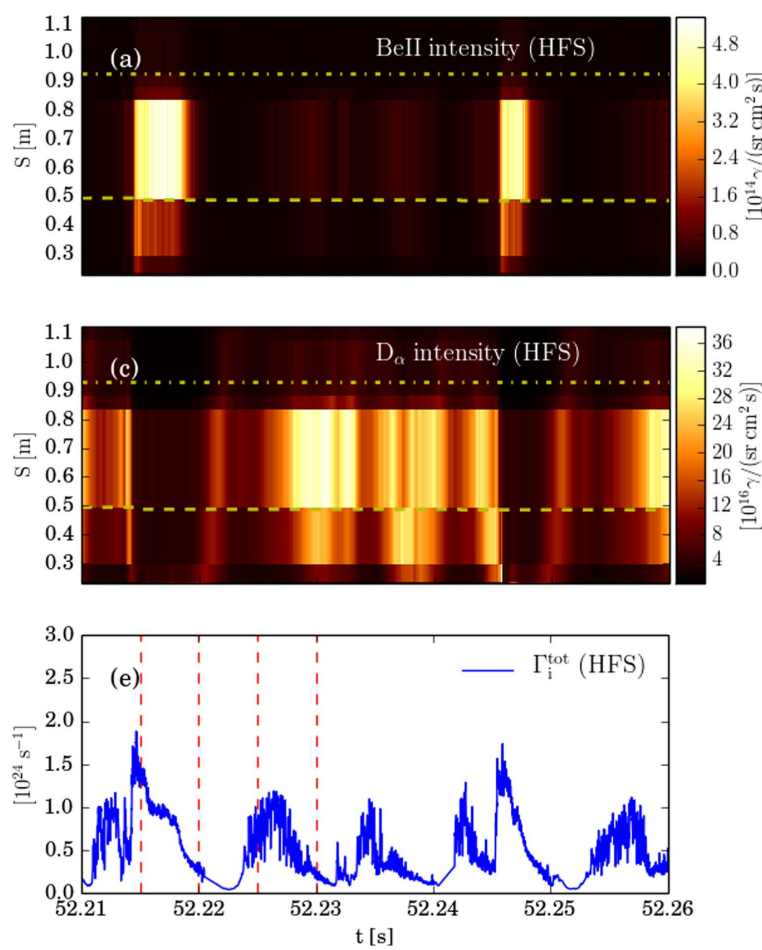
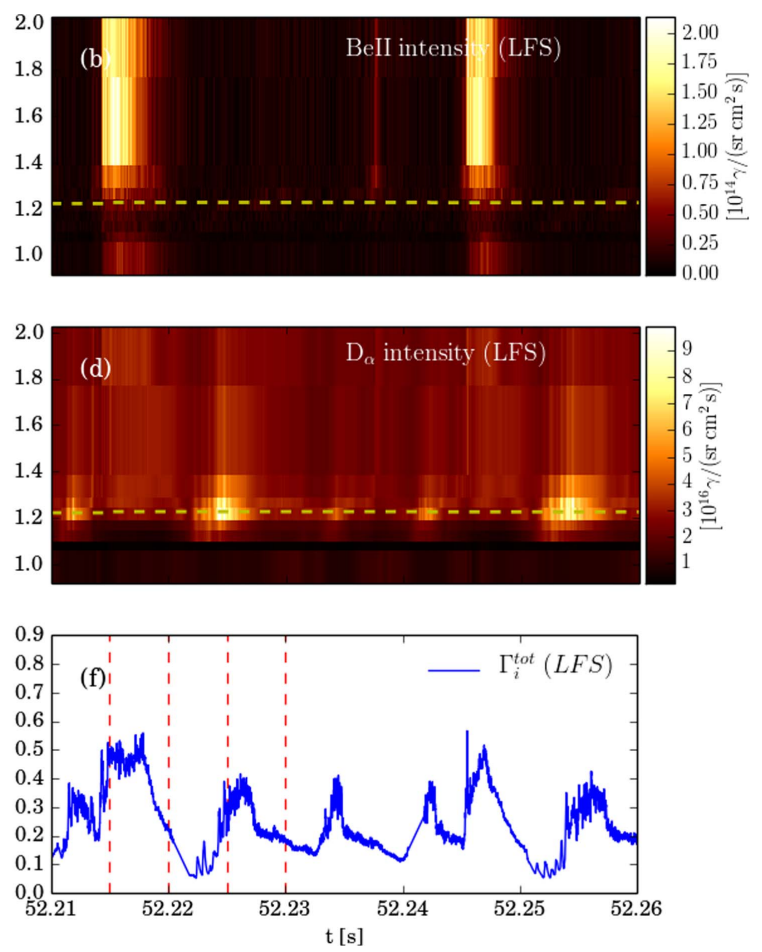

Figure 16. The evolution of BeII $(527 \mathrm{~nm})(\mathrm{a}),(\mathrm{b})$ and $\mathrm{D}_{\alpha}(561 \mathrm{~nm})(\mathrm{c}),(\mathrm{d})$ line intensities during a short period of pulse \#89238 as a function of the $S$-coordinate along the divertor targets (see figure A1) measured using a multi-channel, visible spectrometer (KS3 [31]) viewing the HFS (a), (c), (e) and LFS (b), (d), (f) divertor regions from above and the total ion fluxes $\Gamma_{i}^{\text {tot }}$ (e), (f) at the LFS and HFS targets respectively for pulse \#89238 without $\mathrm{N}_{2}$ seeding. The locations of the strike points (dashed yellow) and the apparent location of the x-point (dot-dashed yellow (HFS only)) are indicated. The times of the emissivity distributions shown in figure 17 are indicated by the red dashed lines in (e), (f).

N) cools the SOL/divertor plasma (see figure 1(d)), leading to rapid detachment from both targets, as indicated by the drop in $\Gamma_{i}^{\text {tot }}$ immediately following the ELM crash.

During these cycles, each drop in ion flux $\Gamma_{i}^{\text {tot }}$ at the HFS target is followed by an increase of the $\mathrm{D}_{\alpha}$ intensity from the inner divertor. There is also an inward movement of the peak $\mathrm{D}_{\alpha}$ intensity, which corresponds to a movement of the emission up the HFS SOL away from the x-point. In contrast, at the LFS the $\mathrm{D}_{\alpha}$ intensity peaks at the strike point, simulataneously with the ion flux to the outer target. Whereas the $\mathrm{D}_{\alpha}$ emission from the outer divertor appears to be associated with recycling, the emission from the inner divertor is perhaps associated with a cool, radiating region which moves in an oscillatory manner along the HFS SOL towards and away from the x-point. This behaviour is investigated in more detail below in terms of changes in the total emissivity distributions.

Tomographic reconstructions of total radiation measurements from the KB5 bolometer system are shown in figure 17 at the four times indicated in figure 16, with a temporal resolution and inter-frame time of $5 \mathrm{~ms}$. This is sufficient to follow a cycle of this periodic detachment behaviour. During the initial frame, at the peak of the ELM crash $\left(t_{\mathrm{ELM}}=\right.$ $51.215 \mathrm{~s}$ ), the peak emission is in the HFS SOL above strike point on the vertical target, while there is a less intense zone of emission in the outer LFS SOL. Following the ELM, during the subsequent, brief detached phase $\left(t_{\mathrm{ELM}}+5 \mathrm{~ms}\right)$, this emission is much reduced but the distribution remains primarily localised to the SOL. Subsequently $\left(t_{\mathrm{ELM}}+10 \mathrm{~ms}\right)$, the peak emission has moved to the $\mathrm{x}$-point region of the confined plasma, while the emission in the SOL is further reduced and the $\Gamma_{i}^{\text {tot }}$ signal indicates temporary re-attachment. In the final frame $\left(t_{\mathrm{ELM}}+15 \mathrm{~ms}\right)$, the ion flux has again reduced, indicating detachment, particularly of the HFS target and the emission from the divertor SOL has again increased.

Similar, oscillations in the detached state have been observed before on ASDEX-U [16] and earlier on JET [15], in L-mode plasmas at medium to high line-average densities, comparable to that in the pulses discussed here, and also during inter-ELM H-mode phases in pulses on ASDEX-U [11]. The frequency of the inter-ELM oscillations reported here $(\sim 100 \mathrm{~Hz})$ is, however, considerably higher than in the earlier JET $(\sim 8 \mathrm{~Hz})$ and ASDEX-U $(\sim 30 \mathrm{~Hz})$ L-mode experiments, and comparable to that of the sub-oscillations reported in [15], which occured during the phase with low $\mathrm{D}_{\alpha}$ emission from the inner divertor. In the recent study of pedestal evolution in JETILW presented in [38], oscillations in the BeII intensity viewing the divertor, at a similar frequency to those discussed here, appear to pace ELMs in pulses at higher $\mathrm{D}_{2}$ fuelling rates. Complementary interpretations of this oscillatory phenomenon are discussed further in section 7.3.

\section{Detachment behaviour with ramped heating power}

Here, the response of the already detached divertor to increased heating power in a $\mathrm{N}_{2}$ seeded pulse is investigated in detail. It is found that, in spite of doubling the heating power, following formation of the x-point MARFE, increased 

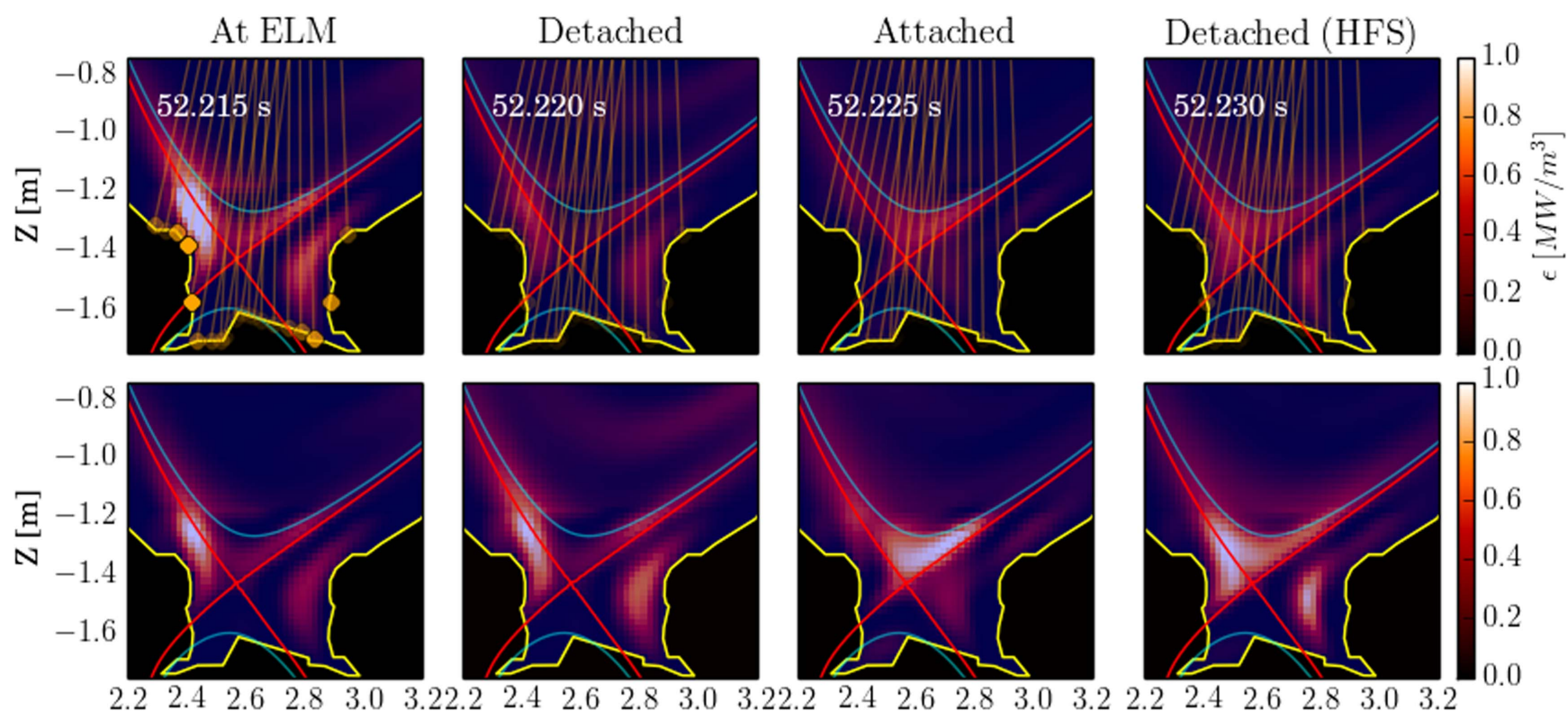

$\mathrm{R}[\mathrm{m}]$

$\mathrm{R}[\mathrm{m}]$

$\mathrm{R}[\mathrm{m}]$

$\mathrm{R}[\mathrm{m}]$

Figure 17. Distributions of the emissivity $\eta(R, Z)$ from tomographic reconstructions of bolometer measurements of the total radiation during pulse \#89238 at the times indicated in figure 16. In the top row the color scale representing the emissivities have the same normalisation, while in the bottom row each plot is normalised to its own maximum value. The smoothing time of the data is $5 \mathrm{~ms}$. The lines of sight of the KS3 visible spectrometer are indicated by the orange lines, with the end points shaded by the normalised BeII (500 nm) line intensity measured by fast photomultipliers (EDG8) [31]. The flux surface at $\psi_{N}=0.98$ is also shown (cyan).

radiation due both to seeded and intrinsic impurities reduces the power to the divertor sufficiently to maintain the detached state. At the higher power, there is significant recombination over an extended region of the divertor SOL, which also broadens considerably.

An important question related to detachment stability is whether the detached state exhibits power hysteresis, i.e. whether more power is required to re-attach the SOL plasma to the targets than the threshold power at which detachment commenced. This could either result from the physics of the plasma within the magnetic geometry of the divertor or from indirect operational issues, e.g. if the increased heating power somehow resulted in more impurity radiation thereby maintaining the detached state.

The effect of doubling the heating power with an initially fully detached divertor is demonstrated by pulse $\# 89244$, the evolution of which is described in section 5.1. In this pulse, the influx of seeded $\mathrm{N}_{2}$ is constantly increasing, so we are not able to make unambiguous statements on the issue of power hysteresis based on data from this pulse. As presented in section 5.2, it is found that increased radiation from both seeded and ELM-sputtered impurities largely offsets the higher power to the divertor $P_{\mathrm{Div}}^{\mathrm{i}-\mathrm{ELM}}$ during the inter-ELM periods, thereby preventing re-attachment of the divertor.

The behaviour of the radiation and recombination fronts determined from the spectral imaging is discussed in section 5.3, paying particular attention to the later, highpower phase. During this phase, investigation of the interELM averaged $j_{\text {sat }}$ profiles in section 5.4 shows that the SOL plasma only partially re-attaches to the inner target, whilst the SOL broadens considerably at the LFS.

\subsection{Effect of increased heating power on detached state}

The evolution of pulse \#89244, in which the input power was increased from $8 \rightarrow 15 \mathrm{MW}$ after $51 \mathrm{~s}$ is shown in figure 18 . This pulse was seeded with $\mathrm{N}_{2}$ at a constant rate of $1.8 \times 10^{22} \mathrm{e} \mathrm{s}^{-1}$ (more than in pulse \#89241 discussed earlier), resulting in a gradually increasing impurity level, with $Z_{\text {eff }}$ increasing from 1.2 to 1.8 over the period from 48 to $54 \mathrm{~s}$ as shown in figure $20(\mathrm{~d})$. Throughout the pulse the lineaveraged density $\left(f_{\mathrm{GW}} \sim 0.8\right)$ and confinement enhancement factor $\left(H_{98, y} \sim 0.8\right)$, shown in figure 18 (c) are quite constant.

During the intermediate, detached phase, there are periods when $f_{\mathrm{ELM}}$ decreases to $\sim 20 \mathrm{~Hz}$ when the pedestal density increases somewhat, which are associated with the formation of the x-point MARFE, as also occurs in the latter phase of pulse \#89241. Later, with the higher input power, the ELM frequency more than doubles while the radiation from the main plasma during the inter-ELM periods $P_{\mathrm{Rad}}^{\mathrm{i}-\mathrm{ELM}}$ remains approximately constant, in spite of the increase in $Z_{\mathrm{eff}}$. This is probably because $T_{\text {e,ped }}$ increases and $n_{\text {e,ped }}$ decreases, resulting in less efficient radiation from the seeded impurity in the pedestal region.

From figure 20(b) it can be seen that at the higher level of $\mathrm{N}_{2}$ seeding the radiated energy during the ELMs $\Delta W_{\mathrm{Rad}}^{\mathrm{ELM}}$ almost equals the ELM energy loss $\Delta W_{\text {ELM }}$, while the deposited energy on T5 $\Delta W_{\text {Dep }}^{\mathrm{T} 5}$ has decreased to $\lesssim 30 \%$ of $\Delta W_{\text {ELM }}$. Hence, the sum of the radiated and deposited energies during the intra-ELM periods exceeds the ELM energy loss, i.e. $\left(\Delta W_{\mathrm{Rad}}^{\mathrm{ELM}}+\Delta W_{\text {Dep }}^{\mathrm{T} 5}\right) / \Delta W_{\mathrm{ELM}} \gtrsim 1$. In section 6 , this is shown to result in cooling of the SOL plasma and a brief period of post-ELM detachment.

The behaviour of the ion fluences $\Phi_{i}^{\text {tot }}$ during the early attached and detached phases are as in pulse \#89241, i.e. the 

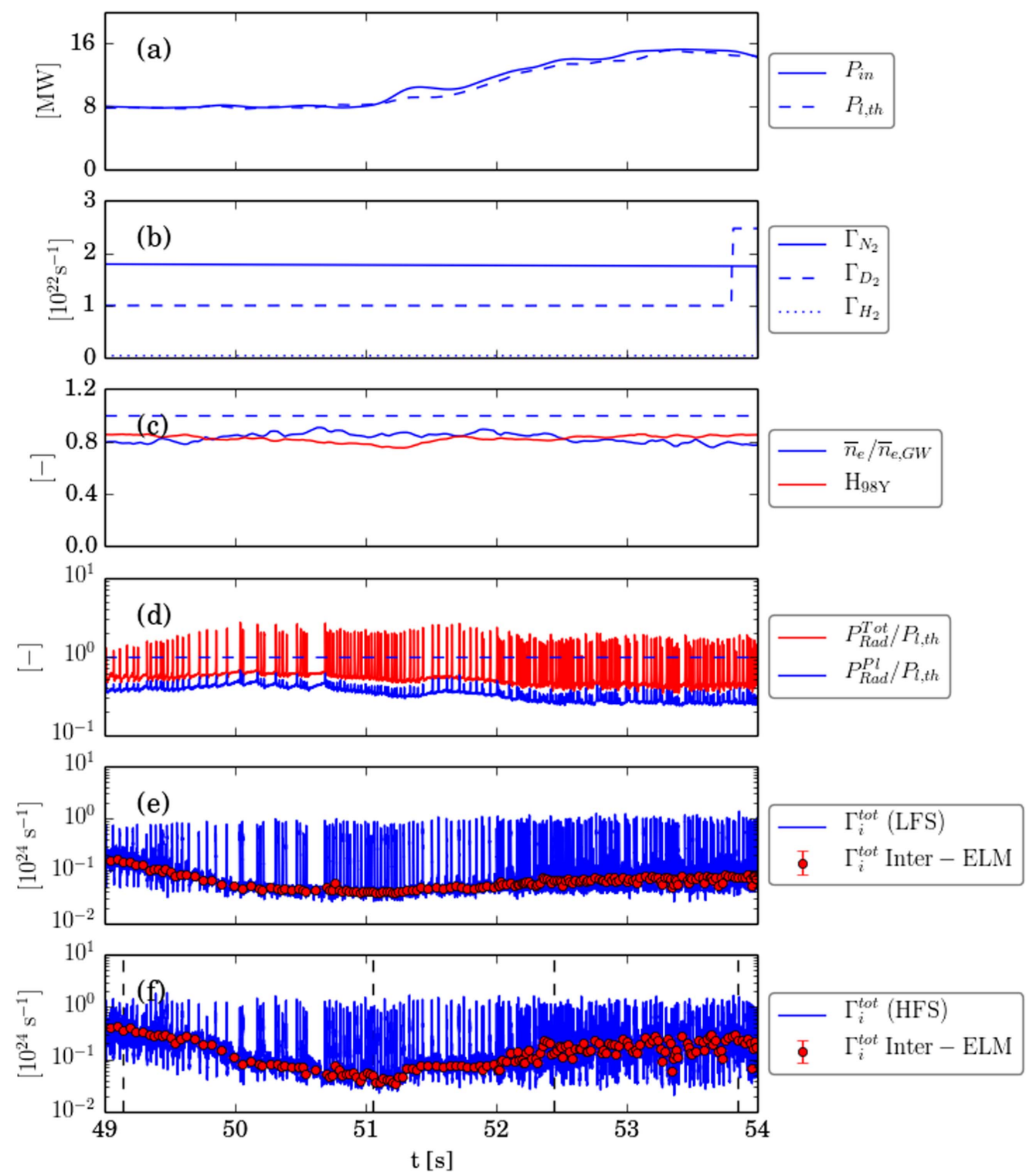

Figure 18. The evolution of JET pulse \#89244 showing: (a) total input power $P_{\text {in }}$ (solid) and the thermal loss power $P_{1, \text { th }}$ (dashed); (b) the $\mathrm{N}_{2}$ seeding rate $\Gamma_{\mathrm{N}_{2}}$ (solid) and the $\mathrm{D}_{2}$ and $\mathrm{H}_{2}$ fuelling rates $\Gamma_{\mathrm{D}_{2}}$ (dashed) and $\Gamma_{\mathrm{H}_{2}}$ (dotted); (c) the Greenwald density fraction $f_{\mathrm{GW}}$ and the $\mathrm{H}$-mode confinement enhancement factor $H_{98, y}$; (d) the fraction of $P_{1, \text { th }}$ radiated in total $f_{\mathrm{Rad}}^{\text {tot }}=P_{\mathrm{Rad}}^{\text {tot }} / P_{1, \text { th }}$ (red) and from the confined plasma $f_{\mathrm{Rad}}^{\mathrm{Pl}}=P_{\mathrm{Rad}}^{\mathrm{Pl}} / P_{1, \text { th }}$ (blue); (e), (f) the total ion fluxes $\Gamma_{i}^{\text {tot }}$ to the LFS (tiles \#2-4) and HFS (tiles \#5-8) targets respectively (blue), with interELM averaged values (red). The times of the emissivty distributions shown in figure 23 are shown by the vertical dashed lines.

fluences during the inter-ELM periods are an order of magnitude larger than the fluences during the ELMs in the attached phase, their ratio subsequently decreasing approximately to unity as the detachment deepens. During the later high-power phase $\geqslant 51 \mathrm{~s}$ ), when the ELM frequency again increases to $\sim 60 \mathrm{~Hz}$, the intra- and inter-ELM fluences remain approximately equal, as shown in figure 20(f).

\subsection{Dependence on divertor input power and radiation}

Initially, when the nominal inter-ELM input power to the divertor $P_{\text {Div }}^{\text {i-ELM }} \sim 2 \mathrm{MW}$ (see figure 19(a)), the divertor plasma is attached to the targets. Soon after the onset of seeding, the inter-ELM ion flux $\Gamma_{i}^{\text {tot }}$ rapidly decreases until
$50 \mathrm{~s}$, after which the plasma detaches from both targets (see figures 19(e), (f)). After the input power is increased, the nominal divertor input power $P_{\text {Div }}^{\mathrm{i}-\mathrm{ELM}}$ approximately doubles. The effect of this is to cause partial re-attachment to the HFS target, while the LFS target remains detached.

The dependence of the ion fluxes $\Gamma_{i}^{\text {tot }}$ at both divertor targets as a function of the divertor input power $P_{\text {Div }}^{\mathrm{i}-\text { ELM }}$ (corrected assuming $f_{\text {def }}=0.15$ ) during the inter-ELM periods is shown in figure 21, where the color indicates the radiated power $P_{\text {Rad }}^{\text {Div }}$ from the divertor region $\left(Z<Z_{\times}\right)$. This evolution can be understood by referring to figure 22 , which shows the radiation from the lower part of the vessel $(Z \leqslant-1.2 \mathrm{~m})$ and the divertor region $\left(Z \leqslant Z_{\times}\right)$as a function of the NII intensity viewing the divertor. 

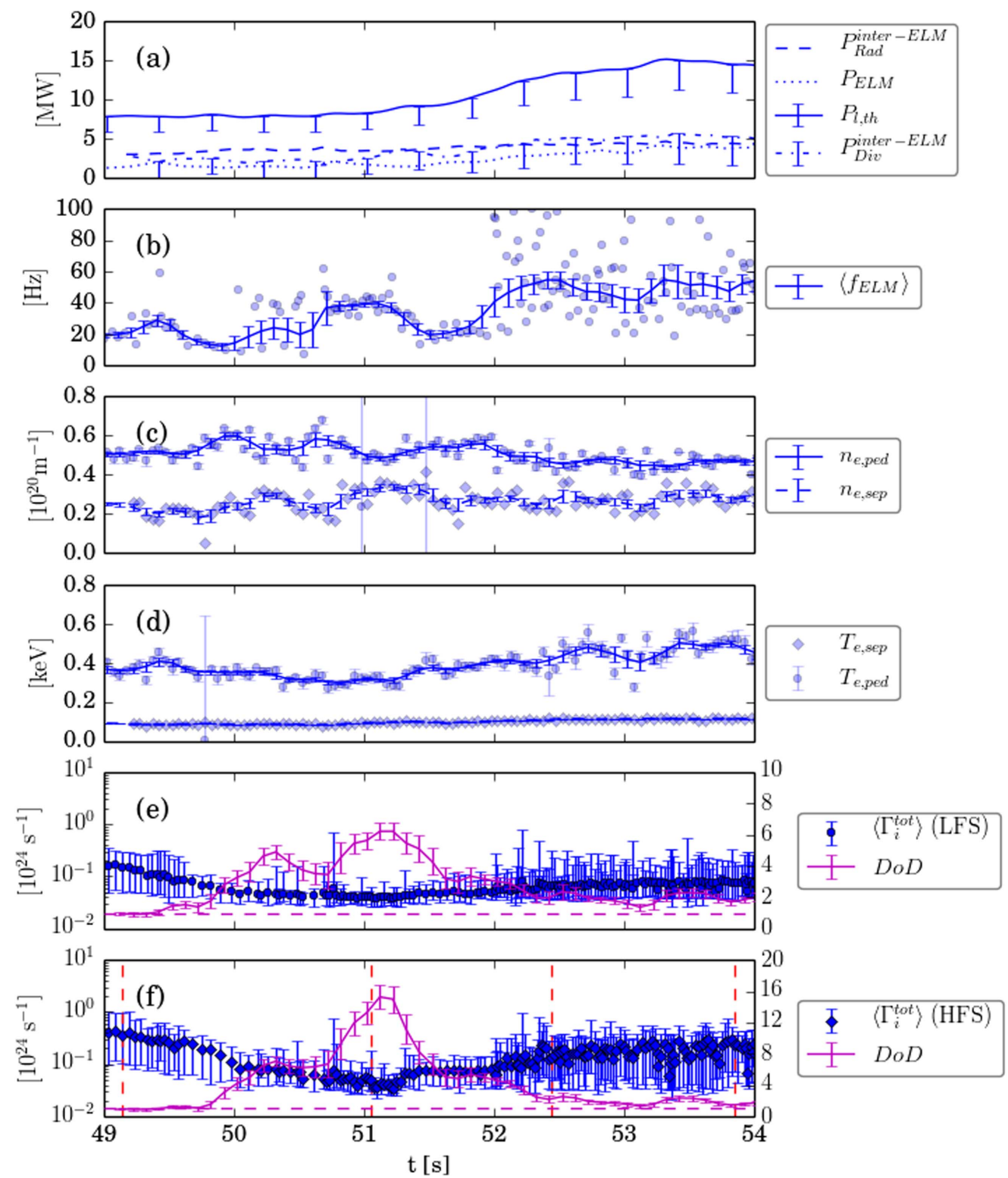

Figure 19. The evolution of relevant parameters of pulse \#89244 with $\mathrm{N}_{2}$ seeding at a constant rate of $\Gamma_{\mathrm{N}_{2}} \sim 1.8 \times 10^{22} \mathrm{e} \mathrm{s}^{-1}$ in which the input power is ramped from $8 \rightarrow 15 \mathrm{MW}$ showing: (a) $P_{1, \text { th }}$ (solid), $\left\langle P_{\mathrm{ELM}}\right\rangle$ (dotted), $\left\langle P_{\mathrm{Rad}}^{\mathrm{i}-\mathrm{ELM}}\right\rangle$ (dashed) and $P_{\mathrm{Div}}^{\mathrm{i}-\mathrm{ELM}}$ (dot-dashed); (b) $f_{\mathrm{ELM}}(\bullet)$ and $\left\langle f_{\text {ELM }}\right\rangle$ (solid); (c) $n_{e \text {,ped }}(\bullet)$ and $n_{e \text {, sep }}(\diamond)$; (d) $T_{e \text {,ped }}(\bullet)$ and $T_{e \text {, sep }}(\diamond)$ and (e), (f) $\left\langle\Gamma_{i}^{\text {tot }}\right\rangle$ to the LFS and HFS targets respectively. The times of the emissivity plots in figure 23 are indicated by the red dashed lines.

With the constant $\mathrm{N}_{2}$ puffing rate, the NII intensity, which is approximately proportional to the $N^{+}$influx, increases throughout the pulse, as does the radiated power from the lower part of the vessel (which includes the x-point region). Once the divertor has detached, however, the radiation from the divertor $P_{\text {Rad }}^{\text {Div }}$ decreases, indicating a loss of impurity retention and a movement of the radiation to the vicinity of the $\mathrm{x}$-point.

During the subsequent high-power phase, as shown in figure 21 , there is an increase in the input power to the divertor $P_{\text {Div }}^{\mathrm{i} \text {-ELM }}$ from $\sim 1 \mathrm{MW}$ to $\sim 1.5 \mathrm{MW}$, with an increase in radiation from the divertor region $P_{\mathrm{Rad}}^{\mathrm{Div}}$ (indicated by the color scale) by $\sim 0.3 \mathrm{MW}$, which is almost sufficient to compensate the increased input power. Although the target ion fluxes $\Gamma_{i}^{\text {tot }}$ increase during this phase to levels similar to those during the intial attached phase, measurements of the $j_{\text {sat }}$ profiles during the inter-ELM phases (see figure 25) show that there is a only a partial re-attchment to the inner target, while the $j_{\text {sat }}$ profile at the outer target is very broad with no clear strike point.

Distributions of total emissivity $\epsilon_{\mathrm{Rad}}(R, z)$ during pulse \#89244 are shown in figure 23 at times (indicated in figure 19(f)) during inter-ELM periods. It can be seen that the peak of the radiation is at the foot of the pedestal, just outside the $\psi_{N}=0.98$ flux surface, to the LFS of the x-point. As shown in figure $20(\mathrm{e})$, the power radiated from this mantle region $\left(\rho_{N}>0.9\right.$ and $\left.z>z_{\times}\right)$increases by $\sim 50 \%$ during the high-power phase, while that from the divertor $P_{\mathrm{Rad}}^{\mathrm{Div}}$ actually more than doubles. 

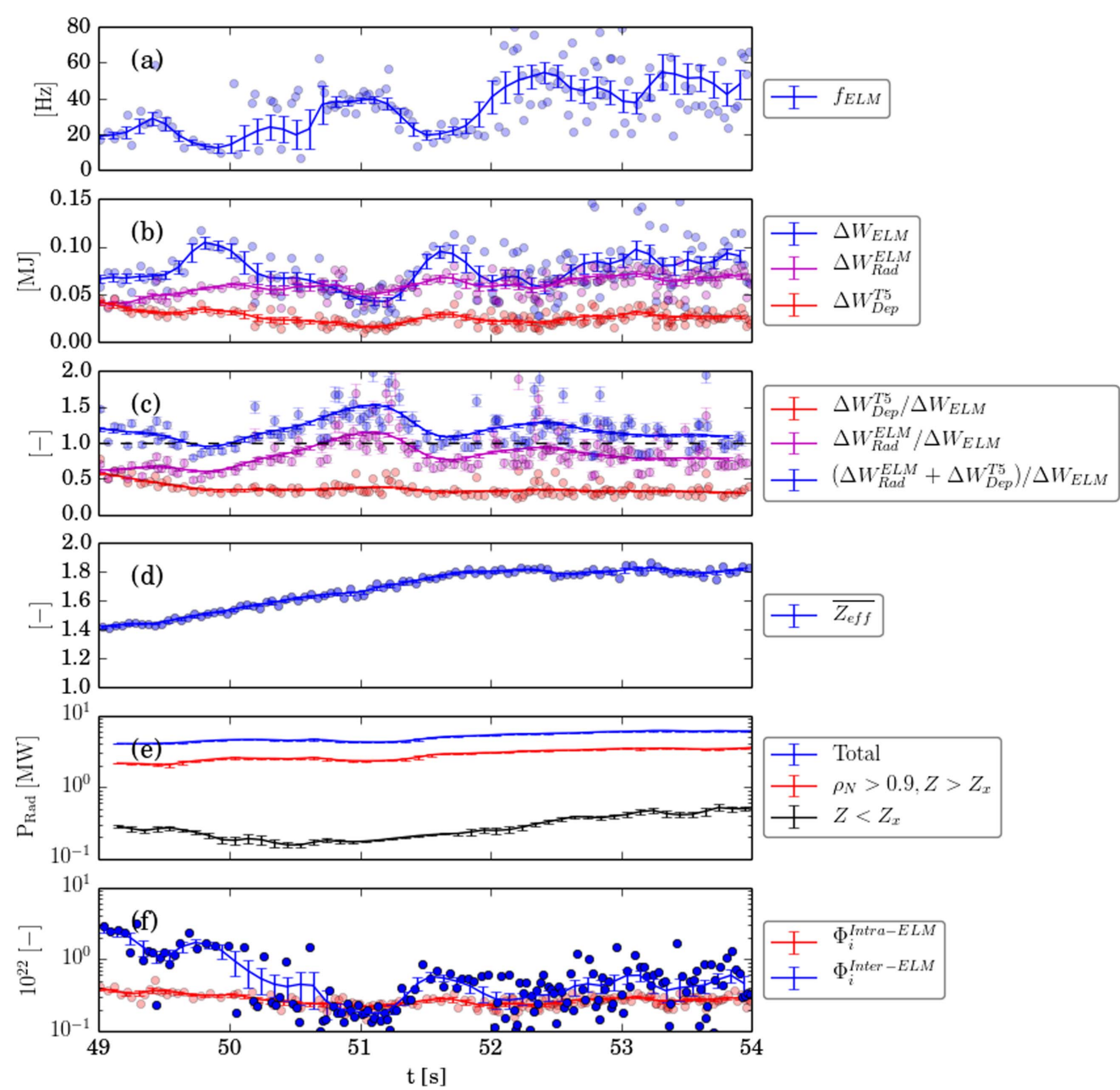

Figure 20. The evolution during pulse \#89244 of: (a) the ELM frequency $\left(\bullet f_{\mathrm{ELM}}=1 / \Delta t_{\mathrm{ELM}}\right.$, and $\left\langle f_{\mathrm{ELM}}\right\rangle=1 /\left\langle\Delta t_{\mathrm{ELM}}\right\rangle$, where $\Delta t_{\mathrm{ELM}}$ is the time between ELM peaks; (b) the energy losses $\Delta W_{\mathrm{ELM}}$ (blue), total radiated energy $\Delta W_{\mathrm{Rad}}^{\mathrm{ELM}}$ (magenta) and the deposited energy $\Delta W_{\mathrm{Dep}}^{\mathrm{T} 5}$ on T5 (red) during the intra-ELM periods; (c) ratios of $\Delta W_{\text {Dep }}^{\mathrm{T} 5} / \Delta W_{\mathrm{ELM}}$ (red), $\Delta W_{\mathrm{Rad}}^{\mathrm{ELM}} / \Delta W_{\mathrm{ELM}}$ (magenta) and $\left(\Delta W_{\mathrm{Rad}}^{\mathrm{ELM}}+\Delta W_{\text {Dep }}^{\mathrm{T} 5}\right) / \Delta W_{\mathrm{ELM}}$ (blue); (d) the line-average, effective ion charge $\overline{Z_{\text {eff }}}$ from visible bremsstrahlung; (e) the inter-ELM radiated power $P_{\mathrm{Rad}}^{\mathrm{i}-\mathrm{ELM}}$ from various regions of the plasma (total-blue, mantle-red, divertor-black); and (f) the total ion fluences $\Phi_{i}^{\text {tot }}=\int \Gamma_{i}^{\text {tot }} \mathrm{d} t$ to both divertor targets during the intra-ELM (red) and interELM (blue) periods. Time-averages of the data points over a sliding Gaussian window of duration $\tau_{\mathrm{sm}}$ of $0.25 \mathrm{~s}$ are represented by the solid lines.

\subsection{Ionisation and recombination front evolution}

From the spectroscopic imaging data for this pulse, shown in figure 24 , it can be seen that the evolution of the radiation and recombination fronts occurs much more rapidly than in the pulse with the seeding ramp \#89241 (see figure 4 of section 2.2) due to the initially lower input power and higher $\mathrm{N}_{2}$ seeding rate. During the later, high-power phase after $51 \mathrm{~s}$, as evident from the maximum NII emissivity from the few ELM-free frames, the thermal front remains close to the $\mathrm{x}$-point, while the region of significant recombination extends from the target well up the divertor leg to within a few $\mathrm{cm}$ of the thermal front. In the frames with ELMs, however, the peak N II emission moves close to the targets, while the peak $\mathrm{D}_{\gamma} / \mathrm{D}_{\alpha}$ ratio remains close to the x-point. The explanation for this different behaviour in response to the ELMs was discussed earlier in section 2.2.

Because the level of seeded $\mathrm{N}_{2}$ is constantly increasing (see figure 22), this pulse does not represent a clean test of the effect of increased heating power alone on detachment. The evolution of the detachment factor $\mathcal{F}_{\text {det }}$, which is shown in figure 24 ( $e, \mathrm{RH}$ axis) gives some indication of the expected effect of the simultaneous change of $\mathrm{N}_{2}$ influx and divertor input power. The intial fractional increase in $\mathcal{F}_{\text {det }}$ of about unity during the low-power phase due to the greater $\mathrm{N}_{2}$ influx causes complete detachment (as in the earlier pulse \#89241). Although the divertor input power $P_{\text {Div }}^{\mathrm{i}-\mathrm{ELM}}$ increases later by $\sim 50 \%$, this is largely offset by the rising $\mathrm{N}_{2}$ level, resulting in a smaller decrease of $\mathcal{F}_{\text {det }}$ to an intermediate value $(\sim 0.7)$ than would be the case with a constant $\mathrm{N}_{2}$ level. (Note that radiation from intrinsic, sputtered impurities, which would also promote detachment, is not taken into account in this measure.)

In conclusion, it appears that, in spite of the increased power into the divertor, the plasma remains detached from the outer target, the thermal front remaining in the vicinity of the $\mathrm{x}$-point. This is consistent with the explanation that additional radiation from both seeded $\mathrm{N}_{2}$ and ELM-sputtered impurities from the SOL reduces the residual power reaching the targets 

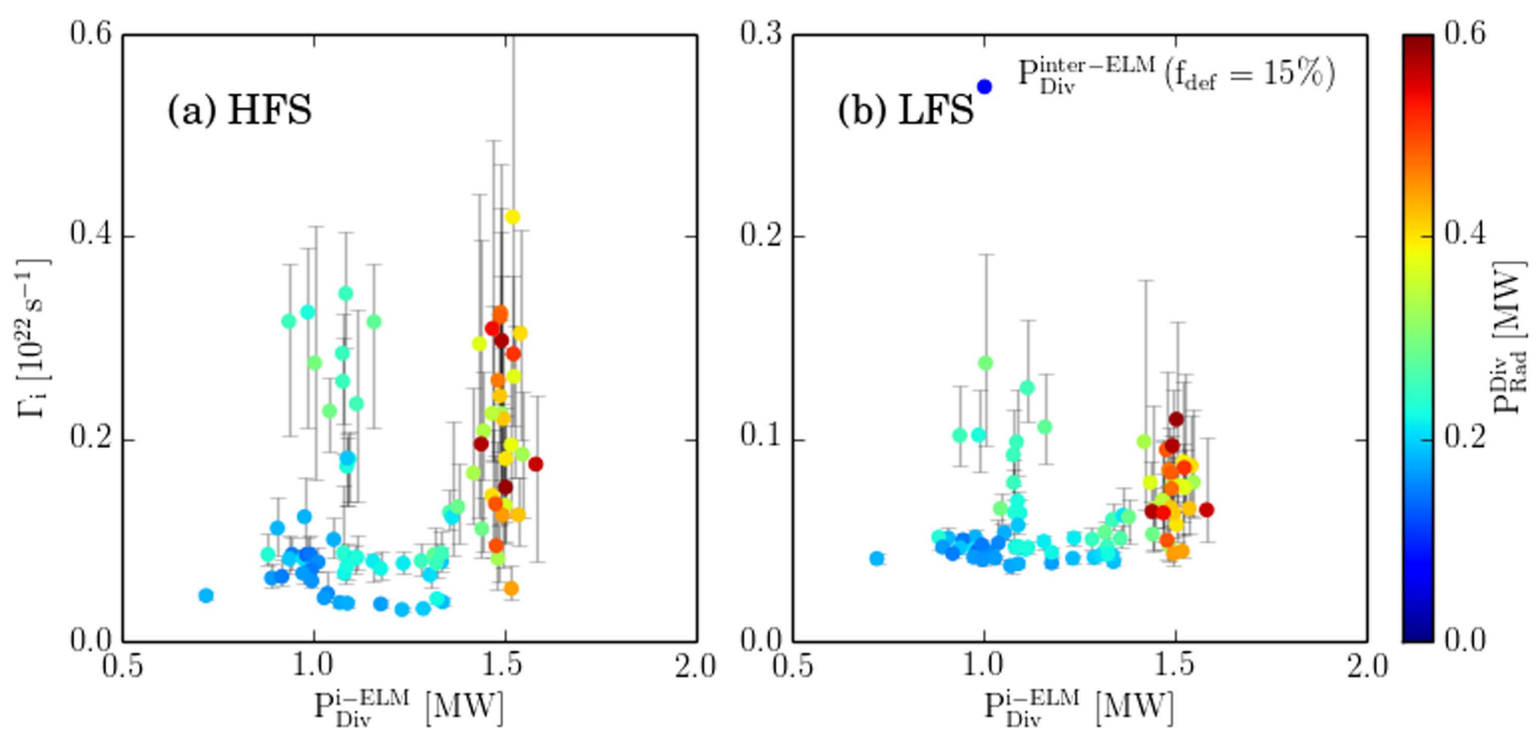

Figure 21. Average values of $\Gamma_{i}^{\text {tot }}$ during inter-ELM periods of pulse \#89244 at the HFS (a) and LFS (b) targets as a function of the corrected power input to the divertor during the inter-ELM periods $P_{\mathrm{Div}}^{\mathrm{i} \text {-ELM }}(\bullet)$ (assuming $f_{\text {def }}=15 \%$ ). The color scale represents the radiated power from the divertor region $\left(Z \leqslant Z_{\times}\right)$.

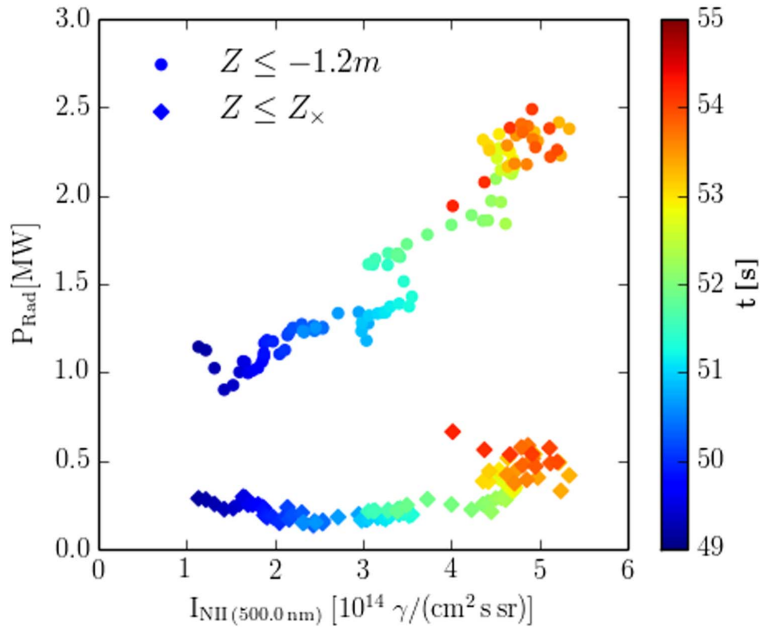

Figure 22. The dependence of the radiated power $P_{\mathrm{Rad}}$ from the lower part of the vessel $(Z \leqslant-1.2 \mathrm{~m})(\bullet)$ and from the divertor region $\left(Z \leqslant Z_{\times}\right)(\diamond)$ on the the NII $(500.0 \mathrm{~nm})$ line intensity measured viewing the divertor region during pulse \#89244.

during the inter-ELM periods sufficiently to maintain detachment, at least from the outer target.

\subsection{Inter-ELM target profiles}

The evolution of $j_{\text {sat }}$ profiles, averaged only over the inter-ELM periods is shown in figure 25. (Note that the effect of the ELMs, which are omitted from the data shown here, is to broaden the $j_{\text {sat }}$ profile considerably.) During the initial attached phase, the peak inter-ELM $j_{\text {sat }}$ is close to the strike point at both the LFS and HFS targets. As level of seeded $\mathrm{N}_{2}$ increases, this decreases until $51.0 \mathrm{~s}$, by which time the plasma is completely detached from both targets. After this time, as the divertor input power begins to increase, so does the total ion flux $\Gamma_{i}^{\text {tot }}$ to both targets (see figures 19(e), (f)). During the later phase, after $52.5 \mathrm{~s}$, when the corrected $P_{\text {Div }}^{\mathrm{i}-\text {-ELM }} \sim 1.5 \mathrm{MW}, j_{\text {sat }}$ only intermittently exhibits a localised peak close to the HFS strike point, indicating partial re-attachment. In contrast, at the LFS the $j_{\text {sat }}$ profile is much broader than during the initial, attached phase with no clear strike point, with most of the interaction occuring in the far SOL.

This broadening of the inter-ELM $j_{\text {sat }}$ profiles during the later high-power phase, without the sharp peak near the strike points, is consistent with detachment, at least of the LFS divertor, as is suggested by the presence of the cold recombining region in the LFS divertor leg evident in figure 24(c). The intermittent inter-ELM interaction with the targets, which is present throughout the pulse, is probably due to filaments ejected from the pedestal or SOL. The presence of this interaction, which is particularly strong with the inner, upper target, produces recycling in this region, which is evident in the $\mathrm{D}_{\alpha}$ and $\mathrm{D}_{\gamma}$ emissivity distributions shown in figures 3(a)(f)). This issue with respect to the broadening of the SOL profiles is discussed further in section 7.5.

\section{Detailed behaviour during the ELM cycle}

Diagnostic data is presented to document the interaction of the ELMs with the divertor plasma and targets in detail. This reveals the sequence of target interaction, sputtered impurity influx, radiation and post-ELM detachment occuring at each ELM and also the interactions due to filaments during the inter-ELM periods at various degrees of detachment.

The detailed behaviour of the interaction of 'typical' ELMs with the target is shown in figure 26 at times during the initial attached (left), detached (centre) and later high-power phases (right) of pulse \#89244 indicated in figure 25. The timing information used for the analysis presented here is derived from the Be II line intensity signal measured viewing the LFS target.

Considering first the initial attached phase $\left(t_{0}=49.113 \mathrm{~s}\right.$, left), before the ELM the $j_{\text {sat }}$ profile data shows the divertor plasma to be strongly attached to both 

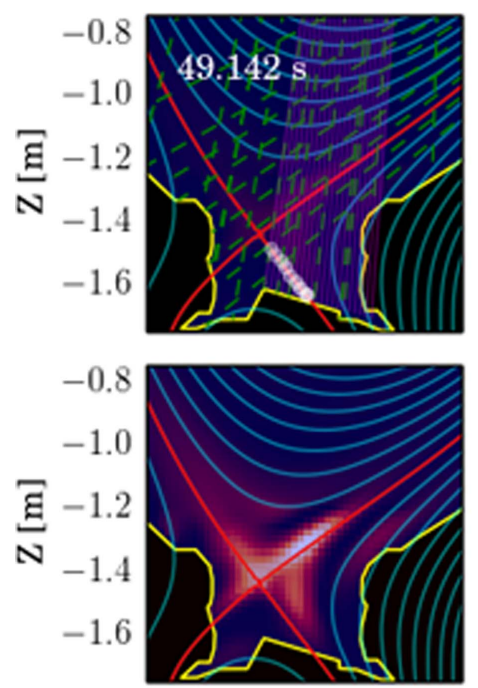

$\begin{array}{lllllllll}2.2 & 2.4 & 2.6 & 2.8 & 3.0 & 3.2 & 2.2 & 2.4 & 2.6\end{array}$

$\mathrm{R}[\mathrm{m}]$
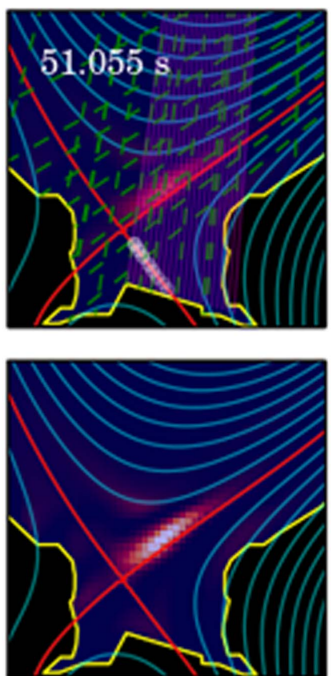

$\mathrm{R}[\mathrm{m}]$
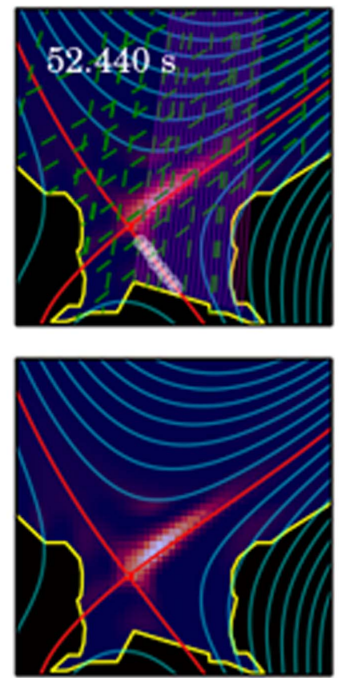

$\mathrm{R}[\mathrm{m}]$
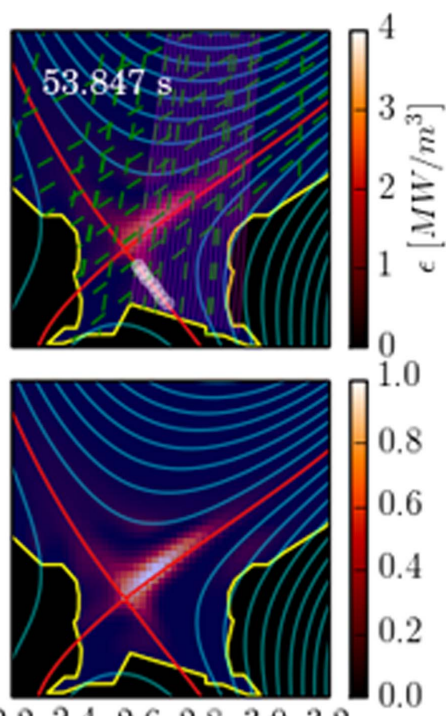

$\begin{array}{llllll}2.2 & 2.4 & 2.6 & 2.8 & 3.0 & 3.2\end{array}$

$\mathrm{R}[\mathrm{m}]$

Figure 23. Distributions of the emissivity $\epsilon_{\text {Rad }}$ from tomographic reconstructions of bolometer measurements of the total radiation during pulse \#89244 at the times indicated in figures 18 and 19. In the top row the color scale representing the emissivities have the same normalisation, while in the bottom row each plot is normalised to its own maximum value. The smoothing time of the data is $5 \mathrm{~ms}$ in all cases. The flux surface at $\psi_{N}=0.98$ is shown in cyan. The opacity of the white markers (top) is linearly scaled to $\left\langle n_{e}^{\text {Stark }}\right\rangle_{\text {Los }}: 0 \rightarrow 5 \times 10^{20} \mathrm{~m}^{-3}$.

targets, with localised interaction close to the strike points, which are located on stack $\mathrm{C}$ of the horizontal T5 target at the LFS and on the vertical T3 at the HFS. During the ELM crash the total ion flux $\Gamma_{i}^{\text {tot }}$ increases by a factor $\sim 2-3$, the $j_{\text {sat }}$ profile spreading outwards considerably beyond the separatrix, while at the strike-point $j_{\text {sat }}$ changes little. The power deposition profile $P_{\mathrm{dep}}^{\mathrm{T} 5}$ over T5/C (measured by IR thermography) also exhibits this broadening on the same timescale, with peak powers reaching almost $100 \mathrm{MW} \mathrm{m}^{-2}$ during the first $\sim 1 \mathrm{~ms}$ of the ELM crash.

After each of the ELMs shown in figure 26 there is a temporary, complete detachment of from the LFS target, indicated by the drop in $j_{\text {sat }}$, which persists until $\Delta t \sim 6 \mathrm{~ms}$ after the ELM peak. This is probably caused by both cooling of the SOL plasma by radiation from sputtered impurities (the radiation from the x-point and divertor region $P_{\mathrm{Rad}}^{\mathrm{Div}} 18$ increases by an order of magnitude during the ELM crashes) and by a reduction of divertor power input $P_{\text {Div }}^{\text {i-ELM }}$ immediately after the ELMs due to the temporary loss of pedestal pressure, which reduces the loss power crossing the separatrix. For the earliest ELM at $49.113 \mathrm{~s}$, the SOL plasma reattaches to the targets again later in the ELM cycle. Note that there is significant power deposited by intermittent filaments during the inter-ELM periods at the level of $P_{\text {dep }}^{\mathrm{T} 5} \sim \mathcal{O}(10) \mathrm{MW} \mathrm{m}^{-2}$.

For the ELM during the phase exhibiting inter-ELM detachment $\left(t_{0}=51.5069 \mathrm{~s}\right.$, centre), while the behaviour during and immediatly after the ELM is much the same as for the earlier ELM, there is almost no sign of re-attachment of the SOL plasma to the targets, with the deposited power $P_{\mathrm{dep}}^{\mathrm{T} 5}$ reaching the target of only a few $\mathrm{MW} \mathrm{m}^{-2}$ in the inter-ELM periods. Later,

18 Note that this is calculated from $P_{\mathrm{Rad}}^{\mathrm{Div}}(\mathrm{TXPN})=P_{\mathrm{Rad}}^{\text {tot }}(\mathrm{TOPI})-$ $P_{\mathrm{Rad}}^{\mathrm{Pl}}(\mathrm{TOBH})$. during the final high power phase $\left(t_{0}=53.5077 \mathrm{~s}\right.$, right $)$ when $P_{\text {Div }}^{\text {i-ELM }} \sim 1.5 \mathrm{MW}$, i.e. about $50 \%$ more than during the earlier attached phase, the SOL plasma remains detached from the LFS target after the ELMs, with only weak, intermittent interaction. At the HFS, however, the plasma partially re-attaches to the target near the strike point, albeit at lower level of $j_{\text {sat }}$ than during the earlier, attached phase.

During the detached and high-power phases, the relative increases of divertor radiation $P_{\mathrm{Rad}}^{\text {Div }}$ (shown in figure $26(\mathrm{~g})$ ), during the ELMs are larger than during the initial attached phase due to the higher level of $\mathrm{N}_{2}$ impurity seeding (see figure 22). Although the amplitude of the ELMs has not increased (see figure 20(b)), their frequency has doubled (see figure 20(a)), hence, there is a greater level of radiation from the sputtered impurities in this phase (see figure 20(e)).

Distributions of total emissivity from the bolometer tomography are shown in figure 27 at four times during the ELM cycle in the early, attached phase. The radiated power $P_{\mathrm{Rad}}^{\text {Div }}$ peaks just after the peak of the BeIl signal $(\Delta t \sim 1 \mathrm{~ms})$, at which time the Be II influx (indicated by the orange markers) can be seen to peak outside the separatrix. The emissivity peaks in the divertor SOL plasma above both targets, although the tomography is unable to resolve its location more precisely. A few ms after this $(\Delta t \sim 4 \mathrm{~ms})$, during the subsequent, temporary detached phase, the emissivity has decreased, while the normalised distributions show that this now peaks in the vicinity of the x-point. Later in the ELM cycle, when the SOL plasma has re-attached to the targets, the emissivity remains low and is primarily localised to the LFS of the $\mathrm{x}$-point. This cycle is evidence for the sputtered impurities entering the confined plasma in the vicinity of the $\mathrm{x}$-point and subsequently radiating there. 

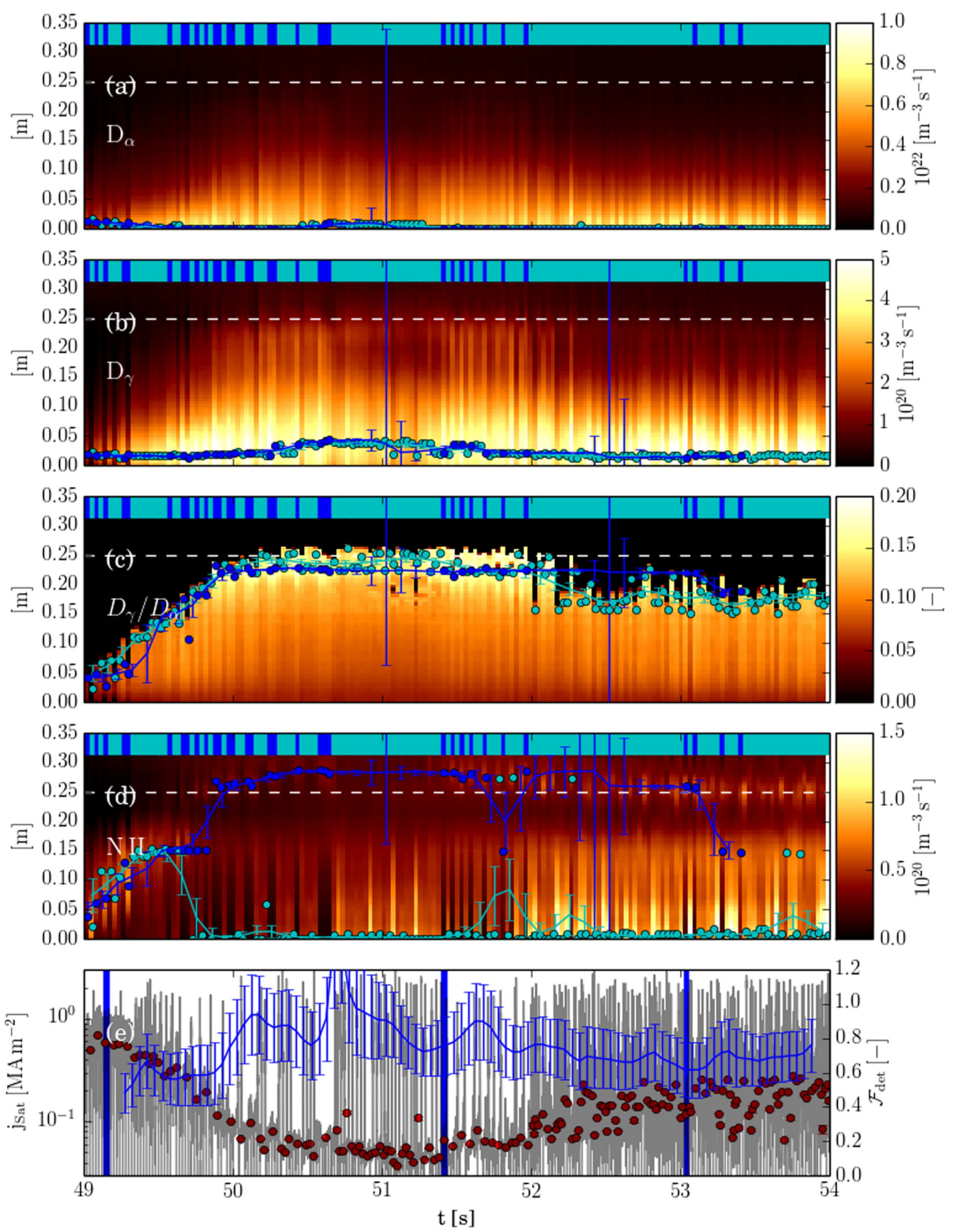

Figure 24. The evolution of line emissivity and emissivity ratio profiles along the flux surface through the S18C target Langmuir probe (shown in figure 5) during pulse \#89244 for: $\mathrm{D}_{\alpha}$ (a) and $\mathrm{D}_{\gamma}$ (b) intensities, the emissivity ratio $\mathrm{D}_{\gamma} / \mathrm{D}_{\alpha}$ (c) and N II(500.4 nm) intensity (d), together with the $j_{\text {sat }}$ signal through the same probe (grey) ( $e$, LH axis) (inter-ELM averaged values shown in red). The combined detachment control parameter $\mathcal{F}_{\text {det }}$ defined in section 1.2, calculated assuming $f_{I} \propto I_{\mathrm{NII}} / n_{u}$, is also shown (blue) (e, RH axis). The locations of the profile maxima for frames with(without) ELMs are shown in (a)-(d) by the cyan(blue) lines respectively. Whether the frames encompass ELMs or are ELM-free is indicated by the upper, cyan/blue markers respectively. The horizontal (dashed-white) lines show the location at the same $z$-coordinate as the x-point.

\section{Discussion}

Here, we draw together the results presented in the previous sections $2-6$, attempting interpret them together in terms of: the evolution of divertor detachment (section 7.1); the conditions promoting divertor detachment (section 7.2); a near-threshold oscillatory state of inter-ELM detachment (section 7.3); the interaction of the ELMs with the divertor plasma (section 7.4); and the effect of increasing the heating power on a fully detached divertor (section 7.5).
The deficiency in the overall energy balance of JET-ILW pulses reported in [9] of up to $25 \%$, caused either by a deficit in the input power or due to some un-measured energy loss, is also borne out by the analysis presented in section 3.4 (see figure 13), in which a $15 \%$ reduction in the loss power $P_{1, \text { th }}$ is required to achieve a consistent power balance of the divertor plasma during the inter-ELM periods. In the following discussion, this fractional deficit $\left(f_{\text {def }}=15 \%\right)$ is assumed when quoting corrected values of the inter-ELM divertor input power $P_{\mathrm{Div}}^{\mathrm{i} \text {-ELM }}$. 

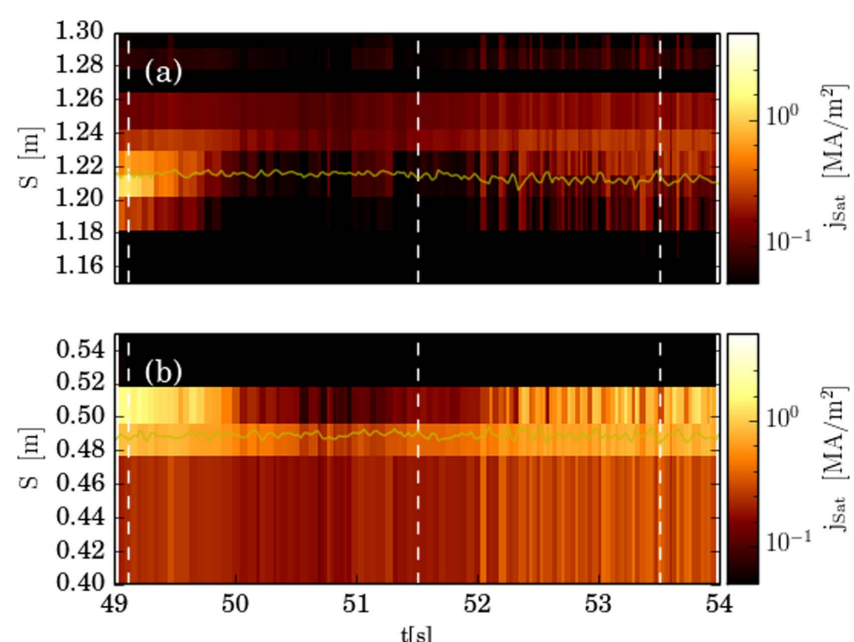

Figure 25. The evolution of the ion saturation current profile averaged during the inter-ELM periods $j_{\text {sat }}^{\text {i-ELM }}$ at the LFS (a) and HFS (b) targets measured by fixed Langmuir probes during pulse \#89244. The $j_{\text {sat }}(S)$ profiles are as a function of the $S$-coordinate measured along the surface of the targets from the HFS to the LFS. The locations of the strike points $S_{\mathrm{sp}}$ from EFIT equilibrium reconstructions are shown (yellow). The vertical dashed lines indicate the times shown in figure 26.

\subsection{Evolution of divertor detachment}

The evolution to detachment in response to $\mathrm{N}_{2}$ seeding is illustrated in section 2.2 by pulse \#89241. As the level of $\mathrm{N}_{2}$ seeding increases, consequent changes in the ELM frequency $f_{\mathrm{ELM}}$, energy losses $\Delta W_{\mathrm{ELM}}$ and radiation from the main plasma $P_{\text {Rad }}^{\text {i-ELM }}$ mutually compensate, resulting in an almost constant input power into the divertor during the inter-ELM periods $P_{\text {Sep }}^{\text {i-ELM }} \sim 2 \mathrm{MW}$ (see figure 14 ), which is apparently close to the detachment threshold.

The initial closeness to the detachment threshold is evident from the observation of oscillations $(f \sim 10 \mathrm{~Hz})$ of the target ion fluxes $\Gamma_{i}^{\text {tot }}$ to both LFS and HFS targets in the early phase $(t \lesssim 50 \mathrm{~s})$ during the inter-ELM periods, which are also present throughout the unseeded pulse \#89238, as shown in figure 16 . In section 7.3 below, we suggest a possible mechanism for these oscillations.

At this divertor input power, an approximate doubling of the $\mathrm{N}_{2}$ influx, which is proportional to the NII line intensity measured viewing the divertor region (see figure 12), results in complete detachment at both targets. The corresponding fractional change in the detachment parameter $\mathcal{F}_{\text {det }}$ as the thermal front moves from the target to the x-point (see figure 4), which is dominated by the change in impurity fraction $f_{I}$, is about unity. This is more than the theoretically predicted detachment window for this parameter quoted in section 1.2 of 0.3 for the LFS divertor. Because of the gross assumptions made, e.g. assuming $f_{I}$ is proportional to the observed NII intensity, the neglect of radiation losses from intrinsic impurities and omission of other physics, e.g. interactions with neutrals, closer agreement is perhaps not expected.

In pulse \#89241, there is little change in the pedestal density and temperature (figure 8(c), (d)) until $52 \mathrm{~s}$, after which there is an abrupt decrease in the ELM frequency (figure $8(\mathrm{~b})$ ) and $n_{e \text {,ped }}$ increases by $\sim 20 \%$. It can be seen from figures $4(\mathrm{c})$, (d) that by this time the radiation front has moved to the $\mathrm{x}$-point, while recombining plasma is present over most of the length of the divertor leg. After this time, as evident from figures 2(c), (d), there is strong radiation near the $\mathrm{x}$-point at the foot of the pedestal, which is consitent with the formation of an x-point MARFE. This corresponds to stage IV of detachment reported in [12]. The reason for the decrease in ELM frequency following MARFE formation is not known. During these phases, the ELM amplitude $\Delta W_{\text {ELM }}$ increases, in spite of an increase in pedestal collisionality, in contradiction to the usually observed dependence [40].

Flux-surface profiles of the NII emissivity and $\mathrm{D}_{\gamma} / \mathrm{D}_{\alpha}$ emissivity ratio at the LFS shown in figures 4(c), (d) provide information on the evolution of the thermal front and recombination region respectively. From onset to full detachment, during the inter-ELM periods the thermal front moves gradually from the target to the x-point, with a region of significant recombination evident over an extended region of the divertor leg between the thermal front to the target. The behaviour at the HFS is difficult to discern from the camera images. There always appears to be strong recycling (high $\mathrm{D}_{\alpha}$ emissivity) and recombination (high $\mathrm{D}_{\gamma} / \mathrm{D}_{\alpha}$ ) close to the vertical target, possibly due to interaction of inter-ELM filaments. As discussed in section 2.3, the ELMs have little effect on the $\mathrm{D}_{\gamma} / \mathrm{D}_{\alpha}$ ratio distribution but do move the maximum NII emission to the target. The effect of the ELMs on the detachment are discussed in section 7.4.

Although from a different pulse, the inter-ELM $j_{\text {sat }}$ profile data shown in figure 25 shows a significant net ion flux to both targets in the outer SOL during the detached phase (50-52 s), particularly to the inner, upper target, which originates from inter-ELM filaments. The energy flux from these filaments on T5 can be seen in the KL9A IR camera data in figure 26(f). Although less evident during the detached than during the attached phase, the inter-ELM power fluxes are still of $\mathcal{O}(1) \mathrm{MW} \mathrm{m}^{-2}$ (in comparison, the noise level after the pulse is $\sim 0.5 \mathrm{MW} \mathrm{m}^{-2}$ ).

Information on the electron density within the recombining plasma in the detached divertor and x-point MARFE is available from Stark broadening of high- $n$ Balmer line emission [41] measured using the KT3A divertor spectrometer $[20,42]$. This is shown in figures 2 and 23 by the opacity of the white markers, which are located where the KT3A lines of sight cross the separatrix ${ }^{19}$. In figure 2 (third column), this data shows that, at full detachment, the density in the recombining plasma along the LFS divertor leg is $\sim 2-3 \times 10^{20} \mathrm{~m}^{-3}$.

The low values of $\mathrm{D}_{\gamma} / \mathrm{D}_{\alpha}$ emmisivity ratios measured in the detached divertor plasma $(\leqslant 0.02)$, implies that other processes than collisional excitation and recombination (radiative and threebody) preferentially populate the $n=3$ level under these conditions. As discussed in [26], possible processes are self-absorption of Lyman- $\beta$ radiation and/or MAR [3]. The mean-free paths of Lyman- $\alpha(-\beta)$ are given by $\lambda_{\text {mfp }} \sim 0.0018(0.012) / N_{0}^{20}$ in $m$ respectively (where $N_{0}^{20}$ is the $D_{0}$ density in $10^{20} \mathrm{~m}^{-3}$ ) [26], i.e.

19 Although it is not possible to determine where the emission is located along the LOS, the high- $n$ Balmer emission is strongly weighted to the region of highest density $\left(\propto n_{e}^{2}\right)$. 

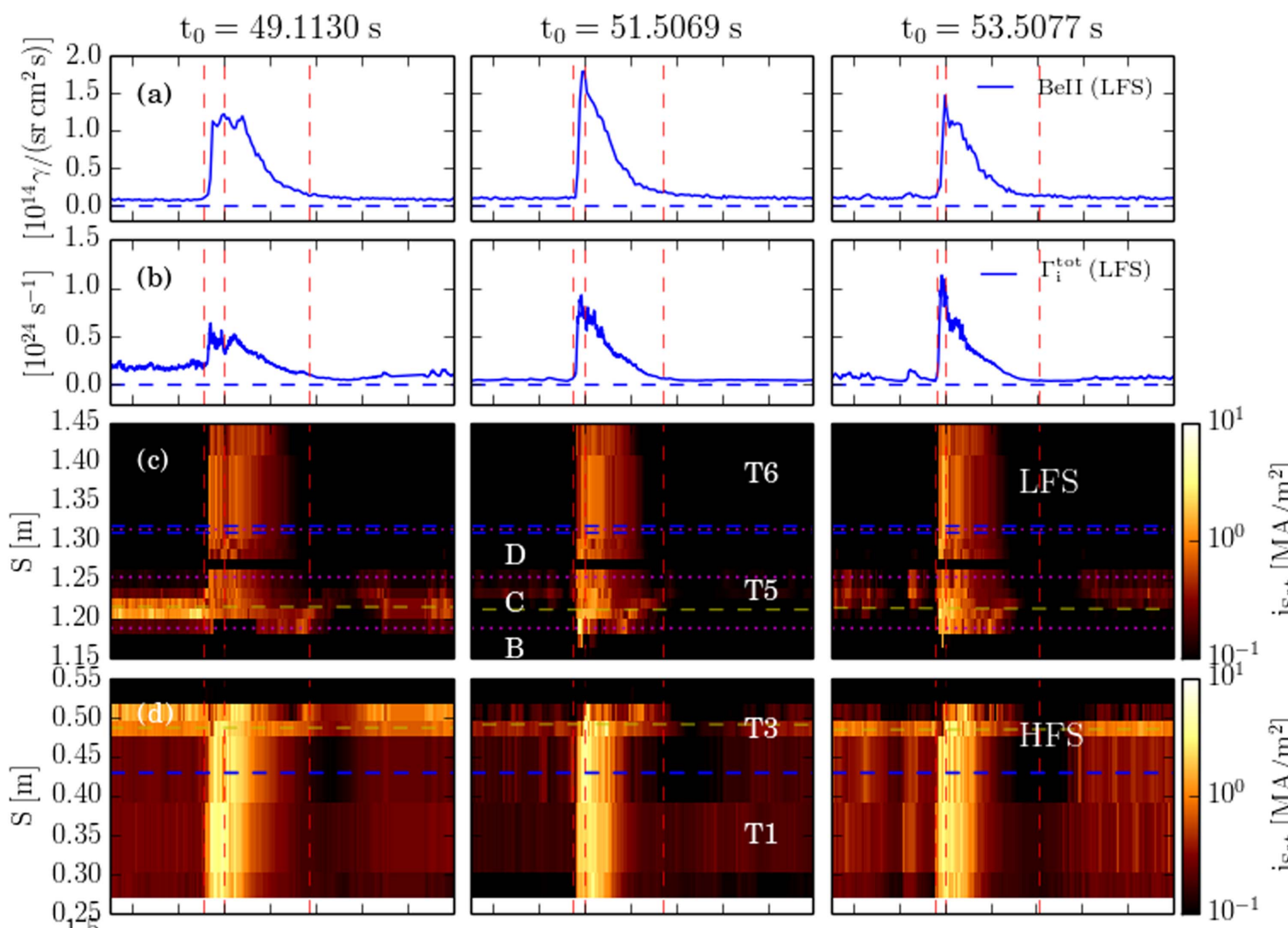

莫
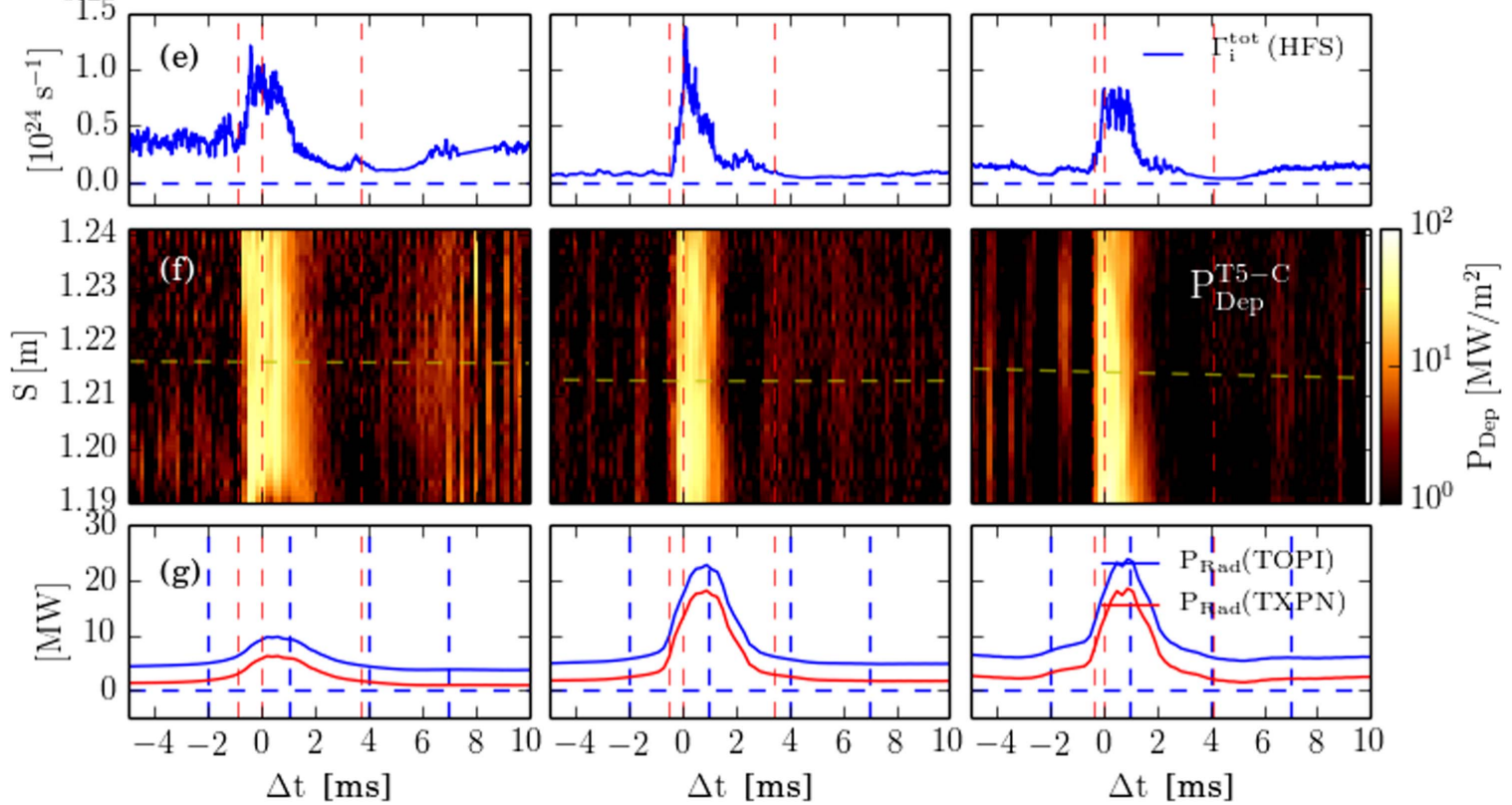

Figure 26. Detailed evolution over typical ELM crashes during the early attached phase (left), the detached phase (centre) and the later higher power phase (right) of pulse \#89244, showing: (a) the BeII intensity (with timing markers-red), (b) the total ion flux $\Gamma_{i}^{\text {tot }}$ and (c) the $j_{\text {sat }}(S)$ profile at the LFS target; (d) the $j_{\mathrm{sat}}(S)$ profile and (e) $\Gamma_{i}^{\text {tot }}$ at the HFS target; (f) the deposited power $P_{\text {dep }}^{\text {T5 }}$ on stack C of T5; and (g) the total radiated power $P_{\mathrm{Rad}}^{\text {tot }}$ (blue) and from the x-point/divertor region $P_{\mathrm{Rad}}^{\mathrm{Div}}(\mathrm{red})$ (low-pass filtered at $200 \mathrm{~Hz}$ ). The locations of the separatrix are shown (yellow-dashed), the locations of the tile edges (blue) and of the T5 stacks (magneta) are shown on the profile plots. The times for the bolometer reconstructions shown in figure 27 are indicated in $(\mathrm{g})$ by the blue-dashed lines.

$\lambda_{\mathrm{mfp}} \sim 1$ and $6 \mathrm{~mm}$ assuming $N_{0}^{20} \sim 2$. Clearly, considering the dimensions of the JET divertor region (see figure A1), it is likely that the recombining plasma is optically thick to these lines, perhaps explaining the low observed values of $\mathrm{D}_{\gamma} / \mathrm{D}_{\alpha}$ emmisivity ratios.
Note that, in an on-going study of detached L-mode plasmas in JET-ILW by Lomanowskij et al [43], high levels of Lyman- $\alpha$ trapping $(\gtrsim 90 \%)$ are found consistent with results of an integrated spectral analysis. As pointed out in [26] and also by Lomanowskij, trapping could substantially 

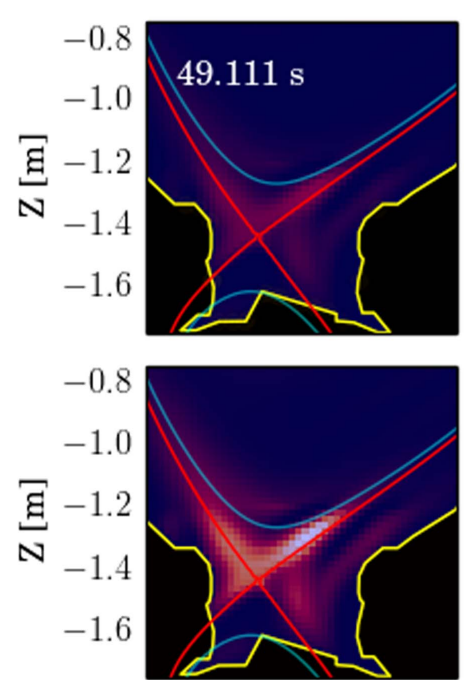

$2.2 \quad 2.42 .6 \quad 2.8$

$\mathrm{R}[\mathrm{m}]$
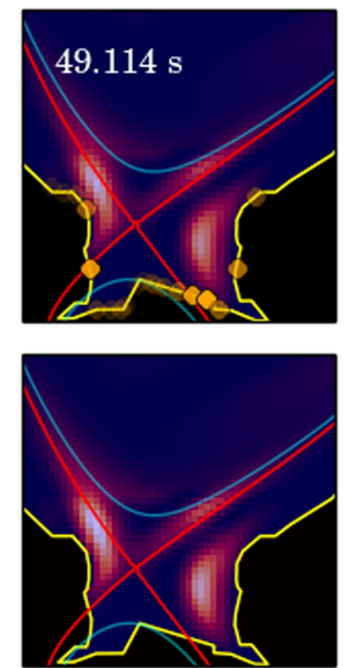
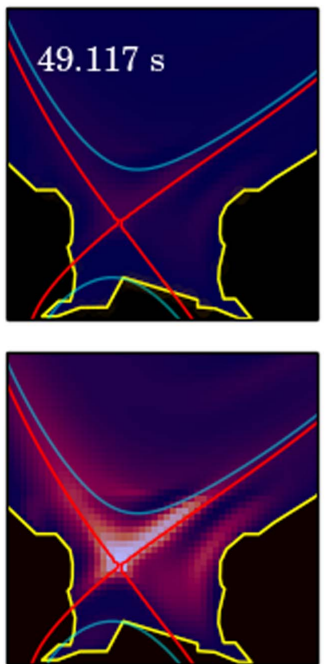

$\mathrm{R}[\mathrm{m}]$
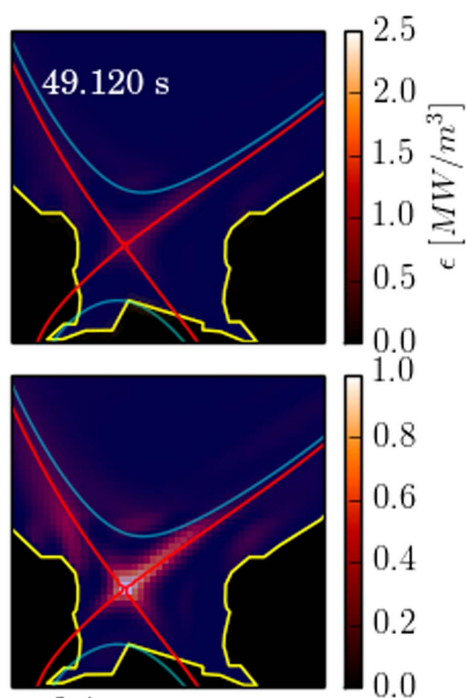

$\begin{array}{lllllll}2.2 & 2.4 & 2.6 & 2.8 & 3.0 & 3.2\end{array}$

$\mathrm{R}[\mathrm{m}]$

Figure 27. Total radiated emissivity distributions $\epsilon_{\mathrm{Rad}}$ from bolometer tomography at the four times $(\Delta t=-2,1,4$ and $7 \mathrm{~ms}$ from the ELM peak) indicated in figure $26((\mathrm{~g})$, left) during the early, attached phase of pulse \#89244. The emissivity is normalised in the bottom row of plots, while the normalised BeII intensity from the EDG8 diagnostic is indicated by the orange dots. The flux surface at $\psi_{N}=0.98$ is also shown (cyan).

reduce the effectiveness of volume recombination as a particle and momentum sink. Further, detailed studies of this kind will certainly be required to reveal the important the atomic and molecular processes in the detached divertor plasma.

\subsection{Conditions promoting divertor detachment}

Here we discuss some general results resulting from our analysis of the conditions promoting divertor detachment that are not covered in the above section.

It can be seen from the dependence of the DoD on the separatrix parameters, i.e. $\Gamma_{i}^{\text {tot }}\left(T_{e \text {,sep }}, n_{e \text {,sep }}\right)$ shown in figure 11 that, for the pulses studied here, full detachment occurs over a considerable range of separatrix (upstream) densities. This variation is much larger than the fractional density detachment windows $\left(\Delta \tilde{n}_{u} \sim\{0.29,0.14\}\right.$ for the LFS and HFS divertors) predicted by analytic theory for this equilibrium [14]. This is because this data represents conditions with a wide range of seeded impurity concentration. It is, therefore, not possible to determine the density detachment window directly from this data. This would be difficult experimentally, requiring the separatrix density to be varied whilst holding $f_{I}$ and $P_{\text {Sep }}$ constant.

The dependence of the target ion fluxes on the pedestal parameters $\Gamma_{i}^{\text {tot }}\left(T_{e \text {, ped }}, n_{e \text {, ped }}\right)$ shown in figure 10 for the LFS and HFS divertors, reveals that the deepest detachment occurs at the highest $n_{e \text {,ped }}$ and lowest $T_{e \text {,ped }}$, when a stable x-point MARFE is present at the foot of the pedestal. Under these conditions the seeded $\mathrm{N}_{2}$ radiates more efficiently from the confined plasma, thereby reducing the power input to the divertor. In the presence of the MARFE, the distributions shown in figures 3(i), (l) exhibit strong NII emission from the LFS SOL plasma above the $\mathrm{x}$-point and recombination below this over most of the LFS divertor leg. However, there does not appear to be significant recombination within the MARFE, where the total radiation peaks (see figure 2).

\subsection{Oscillatory, threshold state of detached divertor}

A fluctuating detached state has been reported previously in L-mode experiments on JET [15] and on ASDEX-U [16]. In both cases, the low-frequency oscillations $(f \sim \mathcal{O}(10) \mathrm{Hz})$ were accompanied by oscillations in the edge plasma density, with the low density phase corresponding to increased $\mathrm{D}_{\alpha}$ emission and higher neutral pressure at the inner divertor and vice versa. Furthermore, variations in the $\mathrm{D}_{\alpha}$ emission from the inner and outer divertors were in anti-phase, as reported here in section 4. In the pulses reported in [15], higher frequency, $(\mathcal{O}(100) \mathrm{Hz})$, sub-oscillations were observed in the $\mathrm{D}_{\alpha}$ intensity only during the phase with low $\mathrm{D}_{\alpha}$ emission from the inner divertor.

Both of these phenomena are not to be confused with the higher-freqency $(\sim 3-8 \mathrm{kHz})$ oscillations in the radiation from the $\mathrm{x}$-point region, measured using A-XUV detectors during inter-ELM periods at stage II of detachment evolution in the ASDEX-U H-mode experiments [11] and also in the L-mode experiments on ASDEX-U [16]. This fluctuating state is associated with high $\mathrm{D}_{\alpha}$ emission from the inner divertor and the formation of a HFS high-density $\left(n_{e} \sim 2.5 \times 10^{20} \mathrm{~m}^{-3}\right)$ (HFSHD) front in the far SOL, which has been observed on ASDEX-U and on JET [44]. As reported in [44], seeding with $\mathrm{N}_{2}$ caused the disappearance of this phenomenon. In the JET experiments, A-XUV diode detectors are not available, so it isn't possible to determine whether such high-frequency fluctuations also occur in such phases.

The detachment oscillations reported here appear to be similar to the slow fluctuations reported previously. Our results also show that this occurs in unseeded conditions at inter-ELM divertor input powers $P_{\text {Div }}^{\mathrm{i} \text {-ELM }}$ close to the threshold 
to initiate detachment. From the total emissivity distributions (figure 17), spectroscopic and target ion flux data (figure 16), it is evident that an oscillation occurs between a state with both targets attached and a state with the HFS target detached and the LFS target partially detached. Based on the data presented in section 4 , we propose a possible mechanism in which the steps below, which correspond to the four times in figure 17 , are repeated cyclicly:

(i) Impurities sputtered from the targets during the attached state intially radiate in the SOL plasma, particularly strongly at the HFS.

(ii) This radiation reduces the net power to the targets, resulting in temporary detachment.

(iii) The impurities migrate to the $\mathrm{x}$-point region where they radiate from the confined plasma, presumably having been ionised to higher ionisation stages.

(iv) This x-point radiation then decays, once the sputtered impurities have been fully ionised or have diffused further into the confined plasma, thereby increasing the net power reaching the targets causing re-attachment.

Note that this mechanism could only occur in a narrow range of divertor input power close to the detachment threshold such that the additional radiation from sputtered impurities is sufficient to induce temporary detachment. Unfortunately, the time resolution of the KT3D spectrometer is insufficient to follow density changes from the Stark broadening measurements.

In a recent study of the physics of pedestal evolution in JET-ILW pulses [38], oscillations in the BeII intensity measured viewing the divertor region (EDG8) are reported at a similar frequency $(150-200 \mathrm{~Hz})$ to those discussed here, which apparently pace the occurrence of ELMs in pulses with high $D_{2}$ fuelling rates of $\Gamma_{D_{2}} \sim 2 \times 10^{23} \mathrm{e} \mathrm{s}^{-1}$, i.e. at similar to the rates used for the pulses in our detachment study.

In [38], the amplitude of high-frequency, broad-band, magnetic oscillations $(150-350 \mathrm{kHz})$ is found to be modulated in phase with the BeII intensity oscillations from both divertors. The authors have proposed an alternative mechanism for the origin of these oscillations to that suggested above, in which the amplitude of filamentary structures due to KBMs in the pedestal, which push out into the SOL and drain energy to the divertor, is modulated by some unknown mechanism, perhaps due to the interaction of flow-shear and magnetic shear with the ballooning mode structure.

Referring to figure 16, during the inter-ELM periods of the pulses from our detachment experiments, the Be II oscillations are much more evident viewing the HFS than the LFS divertor, are in phase with the $\mathrm{D}_{\alpha}$ emission from the HFS divertor and out of phase with the ion flux to both targets $\Gamma_{i}^{\text {tot }}$, which perhaps contradicts the interpretation that they are due to a modulation of the power into the divertor region. Further detailed studies are required of both sets of observations firstly to determine whether they are related phenomena and then, if so, to unravel cause and effect between the cyclic detachment and the modulation of the pedestal turbulence.

\subsection{Interaction of ELMs with the detached divertor}

A key element of the analysis presented here is the ability to classify the data relative to the occurence of the ELMs. This allows the evolution of the inter-ELM detachment to be followed unambiguously and the effect of ELMs on the divertor plasma to be investigated.

Before considering these issues, it should be noted that the ion fluence (time integrated flux) to the targets during the interELM periods usually well exceeds that during the ELMs, except in periods with high-frequency ELMs. It can be seen from the level of fluctuations on the $\Gamma_{i}^{\text {tot }}$ data, e.g. as evident in figure 9, that a substantial contribution to the inter-ELM ion flux is in the form of intermittent bursts, perhaps due to filaments ejected from the pedestal. This figure shows that these are absent immediately after the ELMs, only reappearing after a few ms once the pedestal pressure and/or pressure gradient is restored. This temporary drop in the inter-ELM ion flux could either be caused by a cessation of the flux across the separatrix or else a temporary detachment caused by radiative cooling, the divertor plasma temporarily buffering the ion flux from the filaments.

Returning to the ELM interactions, the most striking data is that from the KL11 imaging system shown in figure 5, where the NII emissivity due only to the ELMs is determined by subtracting subsequent frames with and without ELMs. From this it can be seen that the ELMs interact with the target and divertor plasma in the far SOL, up to $10-20 \mathrm{~cm}$ from the strike point. There is also little change in the emissivity distribution elsewhere in the SOL, as seen from the efficacy of the subtraction. This can perhaps be understood because the timescale for the ELM heat pulse ( $\lesssim 1 \mathrm{~ms}$ ) and subsequent temporary period of detachment $(\sim 5 \mathrm{~ms})$ is much shorter than integration period of the frames $\sim 30 \mathrm{~ms}$, so the measured intensity is dominated by the inter-ELM period. Also, the ELMs are poloidally localised, occupying a small fraction of the plasma surface and are ejected into the far SOL, so they perhaps do not affect the ionisation balance of most of the SOL plasma.

There are two indirect means by which the ELMs do affect the detachment evolution. Firstly, as discussed in section 3.1, the time-averaged ELM power losses from the confined plasma have to be accounted for correctly when determining the net inter-ELM power input to the divertor. Secondly, the ELM-target interaction sputters impurities (W, $\mathrm{Be}, \mathrm{N}_{2}$ ) into the divertor, which can reach the main plasma, thereby affecting the total radiation losses. This is then able to both reduce the input power to and increase the radiation from the divertor, hence deepening (or maintaining) the detached state. It is also the case that this ELM induced radiation causes a temporary cooling and detachment of the divertor plasma as is observed in figure 26.

An important question is the extent to which the ELM energy efflux is 'buffered' by the detached divertor plasma, i.e. the fraction which is dissipated before reaching the targets. This may be by re-ionisation of neutrals in the detached divertor or by impurity radiation. Note that radiation from ELM-sputtered impurities would fail to buffer the initial ELM energy efflux, this occuring after the initial interaction with the target. It can be seen from figure $6(\mathrm{c})$ that $\lesssim 50 \%$ of the ELM 
energy is deposited onto the outer, horizontal $\mathrm{T} 5 / \mathrm{C}$, while $\lesssim 90 \%$ of the energy is radiated, primarily just after the ELM crash. This degree of prompt buffering of the ELM energy loss is consistent with the $\sim 60 \%$ found in studies of both unseeded and Ar seeded type-I ELMy H-mode plasmas in JET reported in [7]. Considering the fact that the energy deposited on the inner target and other PFCs is not measured, the approximate power balance found between the ELM energy loss $\Delta W_{\mathrm{ELM}}$ and the sum of radiated and depositied energies during the ELMs implies that the divertor plasma would be strongly cooled by radiation immediately after the ELMs.

The supposition that prompt radiation from impurities sputtered by the ELMs might cool the SOL is supported by the data shown in figure 26 . Here, it can be seen from the target $j_{\mathrm{sat}}$ profiles (c), (d) that a short period of complete detachment follows a few ms after each ELM, the total divertor radiation peaking about $1 \mathrm{~ms}$ after the peak of the ELM (BeII signal). This behaviour is studied in more detail in figure 7, from which it can be seen that the ELM-crash averaged peak $j_{\text {sat }}$ is less than that during the later, attached inter-ELM period. Immediately after the ELMs (c, d) there is a strong reduction in $j_{\text {sat }}$ due to the occurrence of this brief period of detachment, the depth of which increases with the level of seeding.

Time timescale for the energy deposition on T5 (shown in figure 26(f)) is $1-2 \mathrm{~ms}$, during which $P_{\mathrm{dep}}^{\mathrm{T} 5}$ increases to $\mathcal{O}(100) \mathrm{MWm}^{-2}$. After this initial interaction, $P_{\text {dep }}^{\mathrm{T} 5}$ decreases strongly, indicating that the ELM energy efflux has either been exhausted or has been buffered by radiation. Time-dependent modelling with EDGE2D-EIRENE, presented in [45] for unseeded type-I ELMs in JET-ILW, shows that this timescale for the initial heat deposition is much longer than that of the energy loss at the separatrix $(\lesssim 400 \mu \mathrm{s})$. The assumption of heat-flux limiting factors (0.2) to account for kinetic effects and an ELM crash time several times longer is required to match the timescale of deposition. A similar, strong reduction in $j_{\text {sat }}$ for $\sim 5 \mathrm{~ms}$ after the initial heat pulse as reported here was attributed to an increased particle sink at the target. We suggest instead that this drop in $j_{\text {sat }}$ results from a brief period of detachment induced by radiation from ELM-sputtered impurities.

\subsection{Effect of increased heating power on fully detached state}

The effect of increasing the heating power in a fully detached state is illustrated by pulse \#89244 in which the NBI power increased, almost doubling the input power from 8 to $15 \mathrm{MW}$. This pulse does not provide a demonstration of detachment hysteresis because, in addition to the power ramp, the seeding puff is maintained throughout, resulting in constantly increasing $\mathrm{N}_{2}$ influx and radiated power (see figure 22). Evidence that more input power to the divertor were needed to re-attach the plasma to the target than to initially initiate detachment at otherwise constant conditions would provide a clear demonstration of detachment hyseresis.

At the relatively low initial input power $(\sim 8 \mathrm{MW})$ and high $\mathrm{N}_{2}$ seeding rate, this pulse initially undergoes a rapid evolution to full detachment (see figures 19(e), (f)). Initially, the divertor input power during inter-ELM periods $P_{\text {Div }}^{\mathrm{i}-\text { ELM }} \sim 1 \mathrm{MW}$, which is close to the detachment threshold, hence, only a small increase in impurity radiation is sufficient to cause full detachment. Following detachment, the impurity radiation shifts mainly to the $\mathrm{x}$-point region, indicating a loss of retention in the divertor.

In the intermediate detached phase, there are periods of reduced ELM frequency $\left(f_{\mathrm{ELM}} \sim 20 \mathrm{~Hz}\right.$ ), when the pedestal becomes cooler and more dense, as also observed during the latter phase of pulse \#89241 in the presence of the x-point MARFE. The total emissivity distributions of figure 23 for pulse \#89244 show that an X-point MARFE has also formed in this pulse during this intermediate, detached phase. The KL11 specroscopic imaging data shows a region with significant recombination present over the full extent of the LFS divertor leg from the x-point to the target (figures 24(c), (d)), with the radiation front located still further above the x-point.

After ramping up the input power, almost doubling the nominal loss power $P_{1, \text { th }}$ to $15 \mathrm{MW}$, the corrected input power to the divertor during the inter-ELM periods $P_{\text {Div }}^{\text {i-ELM }}$ only increases modestly from $\sim 1$ to $\sim 1.5 \mathrm{MW}$ (see figure 21 ), most of the additional power having been compensated by increased radiation from the main plasma $P_{\mathrm{Rad}}^{\mathrm{Pl}}$ and timeaveraged ELM losses $\left\langle P_{\mathrm{ELM}}\right\rangle$ due to the higher ELM frequency. As shown by the color scale in figure 21, during this high-power phase, the radiated power from the divertor $P_{\mathrm{Rad}}^{\text {Div }}$ doubles to $\sim 0.5 \mathrm{MW}$, which largely compensates the increased power input to the divertor.

The consequence of the net power to the targets, i.e. $P_{\text {Div }}^{\mathrm{i}-\mathrm{ELM}}-P_{\mathrm{Rad}}^{\mathrm{Div}}$, having barely increased from the low to the high power phases, is that the plasma only partially re-attaches to the inner target and remains detached from the outer target, as shown by the inter-ELM averaged $j_{\text {sat }}$ profiles of figure 25 . At the LFS, $j_{\text {sat }}$ profile is broadened considerably into the far SOL during this latter phase. Furthermore, the radiation front remains near the x-point, with significant recombination extending over the length of the divertor leg to the target.

Even in the presence of the X-point MARFE, the radiated power from the main plasma $P_{\mathrm{Rad}}^{\mathrm{Pl}}$ only increases by $\sim 40 \%$ after doubling the input power, while $f_{\text {Rad }}$ actually decreases. About $40 \%$ of the $P_{\mathrm{Rad}}^{\mathrm{Pl}}$ is radiated from the mantle region $\left(\rho_{N}=0.9-1.0\right)$ and about $70 \%$ this from the x-point MARFE. The increased radiation, particularly from the MARFE region (see figure 22), is due both to the constantly increasing influx of seeded $\mathrm{N}_{2}$ and also impurities $\left(\mathrm{W}, \mathrm{Be}, \mathrm{N}_{2}\right.$ ) sputtered from the targets by the more frequent ELMs. The confinement of the core plasma is, however, hardly affected, with the enhancement factor $H_{98, y}$ remaining at $\sim 0.8$.

Because the level of both seeded and intrinsic impurities is increasing during this pulse as well as the input power, it is not possible to determine whether the detachment exhibits power hysteresis. There is perhaps evidence that the effect of the seeded impurity is largely overcome by the increased power, as shown by the evolution of the combined detachment parameter $\mathcal{F}_{\text {det }}$, which returns to a value characteristic of the earlier attached phase later in the pulse. Therefore, it may be that radiation from sputtered impurities is also contributing to the maintenance of detachment in the high-power phase as that from the seeded $\mathrm{N}_{2}$. 
During the high-power phase, the ELM frequency increases to $f_{\mathrm{ELM}} \lesssim 60 \mathrm{~Hz}$, resulting in almost equal ion fluences to the targets during the ELMs and inter-ELM periods. The inter-ELM ion fluxes $\Gamma_{i}^{\text {tot }}$, shown in figures 19(e) and (f) exhibit large fluctuations as during the early attached phase, which are likely due to filaments ejected from the pedestal or SOL. These are either largely absent during the intermediate phase or buffered by the detached divertor.

The observed spreading of the target $j_{\text {sat }}$ profiles requires enhanced cross-field ion transport in the LFS SOL plasma. A theoretical framework relating filament dynamics and SOL profiles is presented in [46]. In terms of this, it is suggested that such SOL broadening could result from 'clogging' of the parallel particle exhaust by charge exchange collisions with neutrals in the cold recombining plasma [47] of a detached divertor. Under these conditions, considering that radiation trapping is likely to reduce the efficacy of recombination as a momentum sink [43], collisional scattering with neutrals may be an important sink of momentum in the detached plasma.

\section{Conclusions}

By combining data from several of the key JET diagnostic systems, both of the core and divertor plasma, and taking care to classify the data measured during (or including) ELMs or inter-ELM periods, it has been possible to achieve a reasonably comprehensive overview of the divertor detachment behaviour in seeded, type-I ELMy H-mode plasmas in JETILW pulses. It is found that the evolution of the divertor detachment proceeds almost independently of the presence of the ELMs, responding to the control parameters $\left(P_{\mathrm{Sep}}, n_{u}\right.$ and $f_{I}$ ) prevailing during the inter-ELM periods. Because of the short time scale of the ELM heat pulse and because the ELM filaments are ejected into the far SOL and occupy only a relatively small fraction of the plasma surface, they only weakly affect the inter-ELM detachment evolution. They do, however, have an indirect effect by sputtering impurities from the targets, increasing the radiation and thereby reducing the net power input to the divertor during the inter-ELM periods. The ELMs therefore more indirectly than directly affect the evolution of the inter-ELM detachment.

The detachment evolution progresses through the four, well established phases already reported, e.g. in [12], with the fully detached state exhibiting a stable, strongly radiating region of cold, recombining plasma in the x-point region of the pedestal (x-point MARFE). The spectroscopic data is consistent with the divertor plasma being optically thick to Lyman- $\alpha,-\beta$ radiation, which would reduce the efficacy of recombination as a particle and momentum sink. In this fully detached state, almost doubling the input power does not result in full reattachment to both the divertor targets because of increased radiation from both seeded $\mathrm{N}_{2}$ and ELM-sputtered impurities during the high-power phase of the pulse.

Instead of re-attaching, cold, recombining plasma remains throughout the divertor plasma, particularly at the LFS, which causes appreciable broadening of the SOL at the target with no clear strike point. However, under these conditions, the core confinement remains hardly affected by the presence of the radiating mantle. Under these conditions, collisional scattering with neutrals may be an important sink of momentum. It would of course be preferable if the thermal front could be maintained within the divertor rather than progressing to the $\mathrm{x}$-point, however, considering the rather small detachment windows in this divertor geometry, careful, real-time (RT) control of the seeding rate would be required to achieve this. Initial, proof-of-principle experiments on RT detachment control have recently been performed successfully on JET-ILW and are reported in [49].

A concern with this $\mathrm{N}_{2}$ seeded $\mathrm{H}$-mode detachment regime is the continually increasing impurity contamination, e.g. in the higher power pulse, $\overline{Z_{\text {eff }}}$ reaches $\sim 1.8$ within $3 \mathrm{~s}$ of the start of impurity puffing. Comparison with the unseeded pulse, which exhibits a constant $\overline{Z_{\text {eff }}} \sim 1.3$, shows that this is due to the injected $\mathrm{N}_{2}$ and not a build up of intrinsic (W, Be) impurities. Such a level of low- $Z$ impurities would lead to an appreciable fuel dilution in ITER D-T plasmas, e.g. a $Z_{\text {eff }} \sim 2$ due solely to a uniform concentration of $\mathrm{N}^{7+}$ impurity would dilute the fuel ions by a factor $f_{\mathrm{DT}}=\left(Z_{I}-Z_{\text {eff }}\right) /\left(Z_{I}-1\right) \sim 0.83$ and decrease the fusion power by a factor 0.7. It should be noted, however, that in our experiments the $\mathrm{N}_{2}$ seeding was continuous and no attempt was made to control the DoD by limiting the seeding rate to prevent the detachment front reaching the $\mathrm{x}$-point and radiating from the confined plasma.

In the RT detachment control experiments described in [49], the ion saturation current $I_{\text {Sat }}$ from target LPs was used as the sensor and the $\mathrm{N}_{2}$ seeding rate as the actuator. Maintenance of a constant detachment fraction $A_{\text {frac }} \sim 0.5$ (defined in [49] as the ratio of $I_{\mathrm{Sat}}$ to the maximum reached at current roll-over $\left.A_{\text {frac }}=I_{\text {Sat }}(t) / I_{\mathrm{Sat}}\left(t_{\mathrm{RO}}\right)\right)$ was demonstrated for several seconds. As a result, the $\mathrm{N}_{2}$ build-up was reduced, e.g. in pulse $\# 89746, \overline{Z_{\text {eff }}}$ appears to saturate at $\sim 1.5$ by the end of the pulse. Further such RT control experiments, integrating automatic roll-over detection and adaptive gain control, are planned for forthcoming JET campaigns in longer pulses, both with and without strike point sweeping. Results of these experiments should demonstrate whether or not seeding with low- $Z$ impurities can be used to mitigate divertor heat loads without causing an unacceptable level of fuel dilution.

\section{Acknowledgments}

This work has been carried out within the framework of the EUROfusion Consortium and has received funding from the Euratom research and training programme 2014-2018 under grant agreement No 633053 and from the RCUK Energy Programme (grant number EP/P012450/1). To obtain further information on the data and models underlying this paper please contact PublicationsManager@ukaea.uk. The views and opinions expressed herein do not necessarily reflect those of the European Commission. 


\section{Appendix A. JET-ILW divertor geometry}

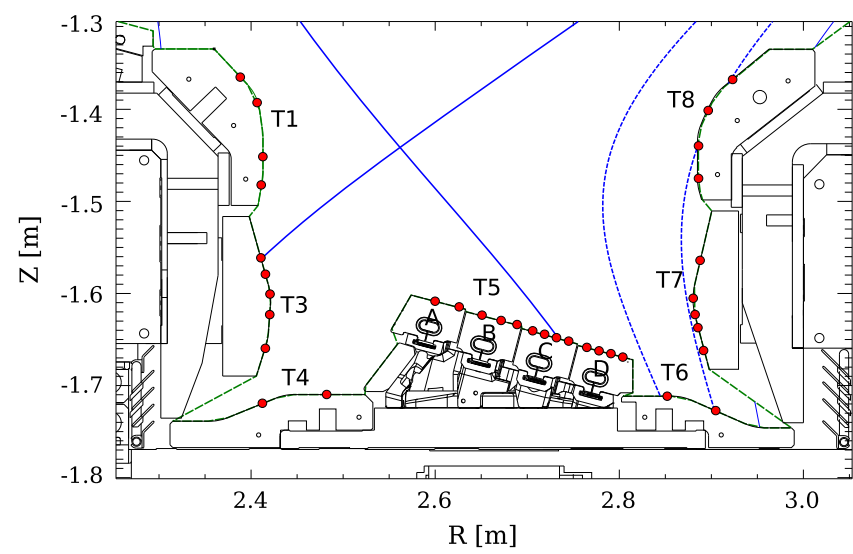

Figure A1. The geometry of the JET divertor with the Mk-2 ITERlike wall showing the locations of the target tiles T1-8 and T5 tile stacks A-D. Also shown are the separatrix and flux surfaces (pulse \#89244 at 50 s) (blue); the locations of the target Langmuir probes (red); and the path of the S-coordinate along the target surfaces (green-dashed).

\section{Appendix B. Description of time-averaging/ integration algorithm}

Here, the algorithm used to calculate the temporally averaged (smoothed) or integrated quantities, e.g. $\left\langle P_{\mathrm{ELM}}\right\rangle$, is described. The object-oriented Python classes for the various diagnostics deliver the measured data in the form of Signal () class objects, which contain as attributes the timebase vector, mean and uncertainty data, units, signal description, etc for the relevant signals. There are several utility methods associate with the Signal () class to perform common operations, e.g. integration over precribed time ranges (e.g. the inter-ELM periods), band-pass filtering, interpolation onto a new timebase, etc. Arithmetic operations are also overridden to perform algebraic operations between pairs of 1D or 2D signals, including error propagation, even on different timebases.

One of these class methods timeAveraged() :

$$
\begin{aligned}
& \text { newSig = rawSig.timeAveraged(tauWin, } \\
& \text { dtNew, normTau } \left.=\text { ' } \mathrm{T} / \mathrm{F}^{\prime} \text {, calcSum }={ }^{\prime} \mathrm{T} / \mathrm{F}^{\prime}\right)
\end{aligned}
$$

returns a new signal object newSig on a new timebase of sampling period dtNew, with the rawSig data temporally averaged or integrated over a sliding Gaussian window function of $1 / e$ duration tausm. If the boolean parameter calcSum is set True the data is integrated rather than averaged, while if the boolean parameter normTau is also set True the integrated data is normalised to tauSm, e.g. to convert an integrated energy to a power signal.

For a 1D signal $x\left(t_{i}\right)$, where the time vector $t_{i}$ need not be regular, this algorithm is performed as follows:

(i) A new, regular time base timeNew $\left(t_{j}\right)$, data vector dataNew $\left(x_{j}\right)$ and uncertainty vector errDataNew $\left(\sigma_{j}\right)$ are created with sample period dtNew over the same period as the raw data. (ii) For input data with mean values $x_{i}$ (data) and standard deviation $\sigma_{i}$ (errData), weights are defined as $w_{i}=1 / \sigma_{i}^{2}$, otherwise $w_{i}=1$.

(iii) At each time point $t_{j}\left(j=\left\{1 \ldots N_{j}\right\}\right)$ in the new time vector:

(a) The Gaussian window function is calculated centred at time $t_{j}$ using:

$$
\mathcal{W}_{j, i}\left(t_{i}\right)=\exp \left\{-\left(\frac{2\left(t_{i}-t_{j}\right)}{\tau_{\mathrm{sm}}}\right)^{2}\right\}
$$

where $\tau_{\mathrm{sm}}$ is the $1 / e$ window duration tauWin.

(b) The normalisation time $\tau_{\text {norm }, j}$ is also calculated from:

$$
\tau_{\text {norm }, j}=\sum_{i=1}^{N_{i}} \mathcal{W}_{j}\left(t_{i}\right) \mathrm{d} t,
$$

where $N_{i}$ is the number of samples in $x_{i}$ and $\mathrm{d} t$ is the sampling interval of the raw data.

(c) The weights are then multiplied by the windowing function $w_{j, i}^{\prime}=w_{i} \mathcal{W}_{j, i}$.

(d) The weighted mean $\mu_{j}$, standard deviation $\sigma_{j}$ and standard deviation of the mean $\overline{\sigma_{j}}$ of the raw data $x_{i} \pm \sigma_{i}$ are then calculated over the windowing function [48]:

$$
\begin{aligned}
\mu_{j} & =\sum_{i=1}^{N_{i}} x_{i} w_{j, i}{ }^{\prime} / \sum_{i=1}^{N_{i}} w_{j, i}{ }^{\prime}, \\
\sigma_{j}^{2} & =\sum_{i=1}^{N_{i}} w_{j, i}{ }^{\prime}\left(x_{i}-\mu_{j}\right)^{2} / \sum_{i=1}^{N_{i}} w_{j, i}^{\prime}, \\
\bar{\sigma}_{j}^{2} & =\sigma_{j}^{2} / \sum_{i=1}^{N_{i}} \mathcal{W}_{j, i} .
\end{aligned}
$$

The resulting, time-averaged datum is hence given by $x_{j}=\mu_{j} \pm \bar{\sigma}_{j}$.

(e) If we wish to integrate the data over the windowing function ( $\operatorname{sumWin}={ }^{\prime} \mathrm{T}$ '), then instead we calulate the output data as:

$$
x_{j}=\mu_{j} \sum_{i=1}^{N_{i}} \mathcal{W}_{j, i}
$$

with uncertainties $\sigma_{j}$, i.e. the output datum is $x_{j} \pm \sigma_{j}$.

(iv) If the integrated data $\left(\operatorname{sumWin}={ }^{\prime} \mathrm{T}\right.$ ') is then to be normalised by the duration of the window (norm$\mathrm{Tau}=' \mathrm{~T} ')$, this is then calculated as $\left\{x_{j} \pm \sigma_{j}\right\}_{\mathrm{norm}}=$ $\left\{x_{j} \pm \sigma_{j}\right\} / \tau_{\text {norm }, j}$.

(v) Finally, a new signal is created on the new time base using the averaged (or integrated) data $\left(x_{j}\right)$ and uncertainty $\left(\sigma_{j}\right)$ vectors:

newSig = Signal(timeNew, dataNew, errDataNew, name = name, units = units)

which is returned by the method. 


\section{ORCID}

J Flanagan (1) https://orcid.org/0000-0003-4179-9616

B Lipschultz 누 https://orcid.org/0000-0001-5968-3684

\section{References}

[1] Shimada M et al 2003 Overview and summary Nucl. Fusion $47 \mathrm{~S} 1$

[2] Stangeby P C 1993 Nucl. Fusion 331695

[3] Krasheninnikov S I et al 1997 Phys. Plasmas 4 1638-46

[4] Matthews G F et al 2011 Phys. Scr. T145 014001

[5] Huber A et al 2013 J. Nucl. Mater. 438 S139-47

[6] Greenwald M 2002 Plasma Phys. Control. Fusion 44 R27

[7] Rapp J et al 2004 Nucl. Fusion 44312

[8] Pasqualotto R et al 2004 Rev. Sci. Instrum. 75 3891-3

[9] Matthews G F et al 2016 Energy balance in JET Proc. 22nd Int. Conf. on Plasma-Surface Interactions in Controlled Fusion Devices (Rome) (https://doi.org/10.1016/j. nme.2016.12.012)

[10] Huber A et al 2012 Rev. Sci. Instrum. 83 D512

[11] Reimold F et al $2015 \mathrm{~J}$. Nucl. Mater. 463 128-34

[12] Reimold F et al 2015 Nucl. Fusion 55033004

[13] Hutchinson I H 1994 Nucl. Fusion 341337

[14] Lipschultz B, Parra F I and Hutchinson I H 2016 Nucl. Fusion 56056007

[15] Loarte A et al 1999 Phys. Rev. Lett. 83 3657-60

[16] Potzel S et al 2013 J. Nucl. Mater. 438 S285-90

[17] Kardaun O J W F 2002 Nucl. Fusion 42841

[18] Loarte A et al 1998 Nucl. Fusion 38331

[19] Koubiti M et al 2002 AIP Conf. Proc. 64567

[20] Meigs A G et al 2013 J. Nucl. Mater. 438 S607-11

[21] Ingesson L C et al 1998 Nucl. Fusion 381675

[22] Fuchs J C et al 1994 Proc. 21st Eur. Conf. on Contr. Fusion and Plasma Phys. vol 18B (Montpellier, EPS, Geneva, 1994) III 1308

[23] Huber A et al 2007 Fusion Eng. Des. 82 1327-34

[24] Fenstermacher M E et al 1997 Rev. Sci. Instrum. 68 974-7

[25] Andersen A and Kak A C 1984 Ultrason. Imaging 6 81-94

[26] Terry J L et al 1998 Phys. Plasmas 5 1759-66
[27] Appel L, Huysmans G, Lao L L, McCarthy P, Muir D, Solano E, Storrs J, Taylor D and Zwingmann W 2006 A unified approach to equilibrium reconstruction $33 \mathrm{rd}$ EPS Conf. on Plasma Phys. (Rome, Italy) number 06

[28] Balboa I et al 2012 Rev. Sci. Instrum. 83 D530

[29] Eich T et al 2007 Plasma Phys. Control. Fusion 49573

[30] Fuchs J C et al 2005 J. Nucl. Mater. 337-9 756-60

[31] Stamp M F, Erents S K, Fundamenski W, Matthews G F and Monk R D $2001 \mathrm{~J}$. Nucl. Mater. 290-3 321-5

[32] Guillemaut C et al 2015 Plasma Phys. Control. Fusion 57 085006

[33] Fundamenski W et al 2006 Plasma Phys. Control. Fusion 48109

[34] Moulton D et al 2013 Plasma Phys. Control. Fusion 55 085003

[35] Ingesson L C 1999 Comparison of methods to determine the total radiated power in JET Technical Report JET-R/99-06 JET Joint Undertaking, Abingdon, UK

[36] Dickenson D et al 2011 Plasma Phys. Control. Fusion 53 115010

[37] Ben Ayed N et al 2009 Plasma Phys. Control. Fusion 51 035016

[38] Bowman C et al 2017 The physics of pedestal evolution in the JET tokamak with the ITER-like wall Nucl. Fusion in preparation

[39] Pitcher C S and Stangeby P C 1997 Plasma Phys. Control. Fusion 39779

[40] Loarte A et al 2002 Plasma Phys. Control. Fusion 441815

[41] Lomanowski B A et al 2015 Nucl. Fusion 55123028

[42] Meigs A et al 2010 Rev. Sci. Instrum. 81 E532

[43] Lowmanowski B et al 2017 Integrated spectroscopic analysis of L-mode detachment in JET-ILW EPS Conf. on Controlled Fusion and Plasma Physics (Dublin) in preparation

[44] Potzel S, Wischmeier M, Bernert M, Dux R and JET-EFDA Contributors $2015 \mathrm{~J}$. Nucl. Mater. 463 541-5

[45] Harting D M, Wiesen S, Groth M, Brezinsek S and JET-EFDA contributors $2015 \mathrm{~J}$. Nucl. Mater. 463 493-7

[46] Militello F and Omotani J T 2016 Nucl. Fus. 56104004

[47] Militello F and Omotani J T 2016 Plasma Phys. Control. Fusion 58125004

[48] Bevington P R and Robinson D K 2003 Data Reduction and Error Analysis for the Physical Sciences 3rd edn (New York: McGraw-Hill)

[49] Guillemaut C et al 2017 Plasma Phys. Control. Fusion 59045001 